\title{
Current status and muon $g-2$ explanation of lepton portal dark matter
}

\author{
Junichiro Kawamura, ${ }^{a, b}$ Shohei Okawa ${ }^{c}$ and Yuji Omura ${ }^{d}$ \\ ${ }^{a}$ Department of Physics, The Ohio State University, \\ Columbus, OH 43210, U.S.A. \\ ${ }^{b}$ Department of Physics, Keio University, \\ Yokohama 223-8522, Japan \\ ${ }^{c}$ Department of Physics and Astronomy, University of Victoria, \\ Victoria, BC V8P 5C2, Canada \\ ${ }^{d}$ Department of Physics, Kindai University, Higashi-Osaka, \\ Osaka 577-8502, Japan \\ E-mail: kawamura.14@osu.edu, okawa@uvic.ca, yomura@phys.kindai.ac.jp
}

ABSTRACT: In this paper, we summarize phenomenology in lepton portal dark matter (DM) models, where DM couples to leptons and extra leptons/sleptons. There are several possible setups: a DM field is either a complex/real scalar or a Dirac/Majorana fermion, and chiralities of leptons that couple to the DM are different according to the spin of the DM. We discuss the prediction of each model and compare it with the latest experimental constraints from the DM detections, the LHC, and the flavor experiments. We also propose simple setups to resolve the discrepancy in the anomalous magnetic moment of muon.

KeYwords: Beyond Standard Model, Cosmology of Theories beyond the SM

ARXIV EPRINT: 2002.12534 


\section{Contents}

1 Introduction 1

2 Lepton portal DM models 3

2.1 Scalar DM models 3

2.2 Fermion DM models 5

3 The LHC limits on extra lepton and slepton $\quad 7$

4 Dark matter physics in the minimal models 9

4.1 Annihilation cross sections 9

$\begin{array}{lll}4.2 & \text { Relic density } & 13\end{array}$

4.3 Indirect detection 14

4.4 Direct detection 16

$\begin{array}{lll}4.5 \text { Current status } & 19\end{array}$

5 Lepton portal DM models for $\Delta a_{\mu} \quad 23$

5.1 Real scalar DM 24

$\begin{array}{ll}5.2 \text { Majorana fermion DM } & 27\end{array}$

6 Summary 30

$\begin{array}{ll}\text { A Analytic expressions } & 31\end{array}$

A.1 Complex scalar DM 31

A.1.1 Annihilation 31

A.1.2 Direct detection $\quad 35$

A.2 Real scalar DM 36

A.2.1 Annihilation 36

A.3 Dirac DM 36

A.3.1 Annihilation 36

A.3.2 Direct detection 36

A.4 Majorana DM 38

$\begin{array}{lll}\text { A.4.1 Annihilation } & 38\end{array}$

$\begin{array}{lll}\text { A.4.2 Direct detection } & 39\end{array}$

B Direct detection limit with non-contact interactions $\quad 39$

$\begin{array}{ll}\text { C Renormalization group equations } & 41\end{array}$

$\begin{array}{lll}\text { C.1 Scalar DM } & 41\end{array}$

$\begin{array}{lll}\text { C.1.1 Complex scalar DM } & 41\end{array}$

C.1.2 Real scalar DM 42

$\begin{array}{lll}\text { C.2 Fermion DM } & 42\end{array}$

C.2.1 Dirac fermion $\quad 42$

$\begin{array}{ll}\text { C.2.2 Majorana fermion } & 42\end{array}$ 


\section{Introduction}

Dark matter (DM) is one of the biggest mysteries in our universe. While evidence of the existence has been accumulated since the first suggestion in 30's, all of them are obtained only through gravitational force. The fundamental nature of DM, e.g. spin and mass, is known very little. This fact has motivated theorists to propose a lot of DM models and many ways to reveal the nature.

A massive elementary particle that weakly interacts with Standard Model (SM) particles has been discussed as a good DM candidate. If the interaction with SM particles is as large as the weak interactions in the SM and the mass is around the weak scale, the thermal relic density of that particle is in agreement with the observed value. This fascinating candidate is called weakly interacting massive particle (WIMP) DM. There are many candidates for WIMP DM in extended SMs; e.g., neutralinos in supersymmetric models [1, 2], Kaluza-Klein particles in extra dimension models [3, 4], inert scalars in extended Higgs models [5-9], and so on. In these models, DM is often accompanied by extra charged or colored particles, which mediate interactions between DM and SM particles. The search for these particles at collider experiments is also a good test for DM models on top of the direct search for DM.

Those extended models are usually motivated by solving theoretical problems in the SM, such as the gauge hierarchy problem, rather than by explaining DM itself. In conventional models, however, recent experimental results severely restrict parameter spaces where DM is explained. In contrast, one can take a more efficient approach to study DM properties by using simplified models once going away from the original motivation. In this approach, an electromagnetically (EM) neutral stable particle is simply added to the $\mathrm{SM}$ as a DM candidate, and the interaction of the DM to the SM sector is introduced in an ad hoc manner. An additional symmetry may also be imposed to guarantee the DM stability. Given a specific setup of a simplified model, one can study DM physics, such as thermal relic density and DM signals, with a limited number of parameters, and examine the viability of the setup. This approach can cover many theoretically motivated models.

In study of simplified models, DM candidates can be classified by its spin and interactions with SM particles. ${ }^{1}$ As the simplest example, let us consider a real scalar DM $X$ which is a singlet under the SM gauge symmetry and interacts with the SM doublet Higgs field $H$ via a scalar quartic coupling, $\lambda_{X} X^{2}|H|^{2}$. This setup is known as the Higgs portal DM model and has been studied well [10-15]. The quartic coupling is responsible for DM thermal production, so that its value can be fixed by the observed relic density. Once the coupling is fixed, one can sharply predict signals of this DM candidate at high-energy collider, direct and indirect detection experiments. As the next-to simplest example, let us introduce a vectorlike charged fermion $F$ in addition to the scalar DM $X$. The scalar DM can be coupled to SM fermions $f^{i}$, where $i$ is a flavor index, via Yukawa couplings, $\lambda_{f}^{i} X \bar{F} f^{i}$, if $F$ has the same gauge charge as $f^{i}$. This kind of model is called the fermion portal DM model $[16,17]$. The signals of the DM in the direct and indirect detection experiments depend on the Yukawa couplings $\lambda_{f}^{i}$. Besides, the models can be tested by the

\footnotetext{
${ }^{1}$ In some cases, it is also important whether DM is self-conjugate or not, as discussed later.
} 
direct search for the vectorlike fermion at high-energy collider experiments and precision measurements of flavor changing processes induced by the Yukawa couplings. In this paper, we shall study this type of model, especially the lepton portal DM model, where the vectorlike fermion has the same gauge charge as the SM leptons.

In the fermion portal DM models, DM annihilation is induced by the $t$-channel exchange of the new vectorlike particle. We can consider several setups: a DM field is either a scalar or a fermion, it is self-conjugate or not, SM fermions coupled to DM are quarks or leptons, and these are further $\mathrm{SU}(2)_{L}$ doublets or singlets. A certain new fermion or scalar fermion (sfermion) field is introduced, so that the portal Yukawa coupling is allowed and the $t$-channel annihilations are turned on. There are many works on the fermion portal models, but most of them focus on only limited setups or particular phenomena. Besides, all these studies were done several years ago. In the present paper, we examine all possible setups in the fermion portal models with all of the latest data from LHC searches, flavor physics and DM physic, including higher-order corrections to DM searches that have not been studied well. Then, we update the current status of each setup. In particular, we concentrate on namely the lepton portal DM models [18, 19], that are expected to evade the strong bounds from direct searches at the LHC and DM direct detection experiments. For the quark portal models, see e.g. refs. [16, 17, 20-29] and references therein.

In this paper, we also study impacts of the lepton portal DM models on precision observables. There is an explicit correlation between these observables and the DM thermal relic density, since both of them are induced via DM-lepton Yukawa couplings. Of these, we especially discuss the anomalous magnetic moment $(g-2)$ of muon. There is a longstanding discrepancy in the muon $g-2[30]$ :

$$
\Delta a_{\mu} \equiv a_{\mu}^{\exp }-a_{\mu}^{\mathrm{SM}}=(268 \pm 76) \times 10^{-11},
$$

where $a_{\mu}^{\exp }$ and $a_{\mu}^{\mathrm{SM}}$ are the experimental result and the SM prediction of $a_{\mu}=(g-2) / 2$ for muon. ${ }^{2}$ It is well-known that the size of the discrepancy is the same order as that of the electroweak (EW) corrections. This suggests a possibility that the discrepancy in $\Delta a_{\mu}$ can be resolved at the same time as the successful thermal DM production in the lepton portal DM models. In refs. [43-46], the authors consider the possibility and figure out that due to strong chirality suppressions, $\Delta a_{\mu}$ cannot be accommodated in the minimal models, where either an $\mathrm{SU}(2)_{L}$ singlet or doublet leptonic mediator is introduced. It is pointed out in refs. $[45,47]$ that the suppression is removed and the discrepancy in $\Delta a_{\mu}$ can be resolved if both mediators are introduced. In these papers, the authors also study the constraints from the collider and direct detection experiments, and discuss the future prospects. Note that these papers only discuss the scalar DM candidates. In our paper, we examine both scalar and fermion DM models, and show that both of the mediators are necessary to reduce the discrepancy, independently of whether the DM field is a scalar or a fermion. At the same time, it turns out that parameter space favored by the muon $g-2$

\footnotetext{
${ }^{2}$ Evaluation of the hadronic contributions to the muon $g-2$ is not conclusive and dominates the theoretical uncertainty [31-41]. In addition, QED NLO corrections to a pion form factor have recently been calculated in ref. [42], where a possibility of the corrections accommodating the discrepancy is examined. The authors have shown that the corrections are too small to diminish the existing discrepancy in the SM.
} 


\begin{tabular}{|c|ccc|}
\hline Name & self-conjugate & spin of DM & spin of mediator \\
\hline real & Yes & 0 & $1 / 2$ \\
complex & No & 0 & $1 / 2$ \\
Majorana & Yes & $1 / 2$ & 0 \\
Dirac & No & $1 / 2$ & 0 \\
\hline
\end{tabular}

Table 1. Classification of DM models based on spins of DMs and mediators.

observation is a bit different in each model. To our knowledge, we identify for the first time which parameter space can accommodate the muon $g-2$ discrepancy in the fermion DM cases. On top of that, we carefully assess the condition for resolving the discrepancy in the lepton portal DM model. We will show that the modification to the minimal models has little impact on the DM physics while it has a big impact on the muon $g-2$ thanks to the large chirality flipping effect. This assessment has not been made in the literature thus far.

This paper is organized as follows. In section 2, we classify the lepton portal DM models, based on the nature of the DM and mediator particles. In this study, DM is assumed to be a singlet under the SM gauge group and to be either scalar or fermion. Then, we study the phenomenology in each model. In section 3, the LHC constraint on each model is analyzed. In section 4, we study the DM physics, i.e. relic density, direct detection and indirect detection, within the minimal models, and summarize the updated status of the models. Contributions to $\Delta a_{\mu}$ in the minimal models are also calculated there, and we reconfirm that the $\Delta a_{\mu}$ is hardly explained. In section 5 , we examine extended models which may be able to explain the discrepancy of $\Delta a_{\mu}$. Section 6 is devoted to summary. In appendix, the details of our calculation concerned with DM physics and renormalization group (RG) equations are shown.

\section{Lepton portal DM models}

We introduce a DM field that is a singlet under the SM gauge group and has spin 0 or $1 / 2$. The scalar DM is denoted by $X$ and the fermion DM by $\chi$. The DM field is also classified according to whether it is self-conjugate or not. For the self-conjugate scalar (fermion) $\mathrm{DM}, X^{\dagger}=X\left(\chi^{c}=\chi\right)$. The self-conjugation nature is important in DM physics. We further introduce a leptonic mediator whose spin is $1 / 2$ (0) for the scalar (fermion) DM. The classification of DM models is shown in table 1. In the following, we shall define scalar and fermion DM models separately.

\subsection{Scalar DM models}

We shall set up scalar DM models in which the DM field is an EM and color neutral real or complex scalar. To realize renormalizable DM couplings to SM leptons, extra leptons are introduced, which are $(\mathbf{2},-1 / 2)$ or $(\mathbf{1},-1)$ under the EW gauge symmetry $\mathrm{SU}(2)_{L} \times \mathrm{U}(1)_{Y}$. The extra leptons should be vectorlike to make the models anomaly-free. The matter contents in the scalar DM models are shown in table 2. In order to stabilize the DM particle, a parity symmetry $Z_{2}$ or global $\mathrm{U}(1)$ symmetry is imposed on the models, under 


\begin{tabular}{|c|cccc|}
\hline fields & spin & $\mathrm{SU}(3)_{c}$ & $\mathrm{SU}(2)_{L}$ & $\mathrm{U}(1)_{Y}$ \\
\hline$L_{R}$ & $1 / 2$ & $\mathbf{1}$ & $\mathbf{2}$ & $-1 / 2$ \\
$L_{L}$ & $1 / 2$ & $\mathbf{1}$ & $\mathbf{2}$ & $-1 / 2$ \\
\hline$E_{R}$ & $1 / 2$ & $\mathbf{1}$ & $\mathbf{1}$ & -1 \\
$E_{L}$ & $1 / 2$ & $\mathbf{1}$ & $\mathbf{1}$ & -1 \\
\hline$X$ & 0 & $\mathbf{1}$ & $\mathbf{1}$ & 0 \\
\hline
\end{tabular}

Table 2. Matter content in the scalar DM model. The subscripts $L, R$ for the fermions represent their chiralities. $L$ and $E$, are vectorlike fermions to respect the anomaly-free condition.

which the DM scalar and the extra leptons non-trivially transform while all the SM fields do not. This symmetry distinguishes the extra leptons $L_{L}\left(E_{R}\right)$ from the corresponding SM ones $\ell_{L}^{i}\left(e_{R}^{i}\right)$. Note that the Higgs portal coupling $|X|^{2}|H|^{2}$ is always allowed in the scalar models. It could have some impacts on the DM annihilation and direct detection cross sections. In this study, we assume that the Higgs portal coupling is negligibly small, and do not consider any combinational effects with it, to highlight the phenomenology induced by the lepton portal couplings.

We introduce a relevant part of the Lagrangian in the scalar DM model. The mass terms for the vectorlike leptons are given by

$$
-\mathcal{L}_{S, \text { mass }}=m_{L} \bar{L}_{L} L_{R}+m_{E} \bar{E}_{L} E_{R}
$$

The Yukawa interactions involving the vectorlike leptons are given by

$$
-\mathcal{L}_{S, \text { Yukawa }}=\lambda_{L}^{i} \bar{\ell}_{L}^{i} X L_{R}+\lambda_{R}^{i} \bar{E}_{L} X^{*} e_{R}^{i}+\kappa \bar{L}_{L} H E_{R}+\tilde{\kappa} \bar{E}_{L} \tilde{H} L_{R}+\text { h.c. },
$$

where $\ell_{L}^{i}$ and $e_{R}^{i}$ are the $\mathrm{SU}(2)_{L}$ doublet and singlet leptons in the $\mathrm{SM}$ and $\tilde{H} \equiv i \sigma_{2} H^{\dagger}$. The index $i=1,2,3$ runs over the SM three generations. The first two terms are the portal couplings of the DM to SM leptons. The latter two terms in eq. (2.2) generate the mass mixing between the vectorlike charged leptons after the EW symmetry breaking. The mass matrix is given by

$$
\left(\bar{E}_{L}^{\prime} \bar{E}_{L}\right)\left(\begin{array}{cc}
m_{L} & \tilde{\kappa} v_{H} \\
\kappa v_{H} & m_{E}
\end{array}\right)\left(\begin{array}{c}
E_{R}^{\prime} \\
E_{R}
\end{array}\right)
$$

where $v_{H} \equiv\left\langle H_{0}\right\rangle$ is the Higgs vacuum expectation value (VEV). The primed field $E_{L}^{\prime}\left(E_{R}^{\prime}\right)$ is the charged component in the doublet vectorlike lepton $L_{L}\left(L_{R}\right)$. We define the mass eigenstates as

$$
\left(\begin{array}{c}
E_{R}^{\prime} \\
E_{R}
\end{array}\right)=\left(\begin{array}{cc}
c_{R} & s_{R} \\
-s_{R} & c_{R}
\end{array}\right)\left(\begin{array}{c}
E_{R_{1}} \\
E_{R_{2}}
\end{array}\right), \quad\left(\begin{array}{c}
E_{L}^{\prime} \\
E_{L}
\end{array}\right)=\left(\begin{array}{cc}
c_{L} & s_{L} \\
-s_{L} & c_{L}
\end{array}\right)\left(\begin{array}{c}
E_{L_{1}} \\
E_{L_{2}}
\end{array}\right),
$$

where $c_{X}, s_{X}(X=L, R)$ satisfy $c_{X}^{2}+s_{X}^{2}=1$. The left- and right-handed fermions can be combined to Dirac fermions as $E_{a} \equiv\left(E_{L_{a}}, E_{R_{a}}\right)$, where $a=1,2$. Their masses are denoted by $m_{E_{1}}$ and $m_{E_{2}}$. In the mass base, the Yukawa couplings involving the DM and vectorlike leptons $E_{1,2}$ are given by

$$
\mathcal{L}_{S, \text { Yukawa }} \supset X \bar{e}_{i}\left[\left(\lambda_{L}^{i} P_{R} c_{R}-\lambda_{R}^{i *} P_{L} s_{L}\right) E_{1}+\left(\lambda_{L}^{i} P_{R} s_{R}+\lambda_{R}^{i *} P_{L} c_{L}\right) E_{2}\right]+\text { h.c. }
$$

where $e_{i}$ is a SM charged lepton in the $i$-th generation. 


\begin{tabular}{|c|cccc|}
\hline fields & spin & $\mathrm{SU}(3)_{c}$ & $\mathrm{SU}(2)_{L}$ & $\mathrm{U}(1)_{Y}$ \\
\hline$\widetilde{L}$ & 0 & $\mathbf{1}$ & $\mathbf{2}$ & $-1 / 2$ \\
$\widetilde{E}$ & 0 & $\mathbf{1}$ & $\mathbf{1}$ & -1 \\
\hline$\chi$ & $1 / 2$ & $\mathbf{1}$ & $\mathbf{1}$ & 0 \\
\hline
\end{tabular}

Table 3. Matter content in the fermion DM model.

The model would resolve the discrepancy in the muon anomalous magnetic moment. The new physics contribution to $\Delta a_{\mu}$ in the scalar DM model is given by [48-50],

$$
\begin{array}{r}
\Delta a_{\mu}=\frac{m_{\mu}}{16 \pi^{2} m_{X}^{2}}\left[\left(c_{R}^{2}\left|\lambda_{L}^{\mu}\right|^{2}+s_{L}^{2}\left|\lambda_{R}^{\mu}\right|^{2}\right) m_{\mu} F_{f}\left(x_{1}\right)+c_{R} s_{L} \operatorname{Re}\left(\lambda_{L}^{\mu} \lambda_{R}^{\mu}\right) m_{E_{1}} G_{f}\left(x_{1}\right)\right. \\
\left.+\left(s_{R}^{2}\left|\lambda_{L}^{\mu}\right|^{2}+c_{L}^{2}\left|\lambda_{R}^{\mu}\right|^{2}\right) m_{\mu} F_{f}\left(x_{2}\right)-c_{L} s_{R} \operatorname{Re}\left(\lambda_{L}^{\mu} \lambda_{R}^{\mu}\right) m_{E_{2}} G_{f}\left(x_{2}\right)\right]
\end{array}
$$

where $x_{i}=m_{E_{i}}^{2} / m_{X}^{2}$. The loop functions are defined as

$$
F_{f}(x)=\frac{2+3 x-6 x^{2}+x^{3}+6 x \log x}{6(1-x)^{4}}, \quad G_{f}(x)=\frac{3-4 x+x^{2}+2 \log x}{(1-x)^{3}} .
$$

With $m_{X} \sim \mathcal{O}(100 \mathrm{GeV})$, the current discrepancy can be explained only if the chirality-flip effect proportional to the vectorlike lepton mass $m_{E_{1,2}}$ is sizable. This arises only in the model with the non-vanishing mass mixing, i.e. $c_{R} s_{L} \neq 0$ and/or $c_{L} s_{R} \neq 0$.

A discrepancy in the electron anomalous magnetic moment $\Delta a_{e}$ is also reported [51] recently, although it is less significant than that of the muon. We expect that $\Delta a_{e}$ can be explained in the lepton portal DM models instead of $\Delta a_{\mu}$. These anomalies, however, cannot be explained simultaneously, since the lepton flavor violating (LFV) decay $\mu \rightarrow e \gamma$ is induced just as the portal Yukawa coupling to the electron is turned on. ${ }^{3}$ We do not pursue this possibility in this paper. The simultaneous explanation for both anomalies are studied in refs. [55-64].

Throughout this paper, the word "minimal" refers to the setups in which either doublet or singlet leptonic mediator exists and the other is absent. In the model Lagrangian introduced in this section, the minimal setups are therefore obtained by simply neglecting either $L$ or $E$. The chirality-flip effects to $\Delta a_{\mu}$ are absent in these setups. As mentioned above, we need move on to the non-minimal setup that contains both the vectorlike leptons, to address the discrepancy in $\Delta a_{\mu}$.

\subsection{Fermion DM models}

The fermion DM can be Majorana or Dirac. In the fermion DM models, mediators are complex scalars and are $(\mathbf{2},-1 / 2)$ or $(\mathbf{1},-1)$ under the EW symmetry. The $\mathrm{SU}(2)_{L}$ doublet (singlet) mediator, named slepton, is denoted by $\widetilde{L}(\widetilde{E})$. The matter content is shown in table 3. As in the scalar DM models, the minimal setups are obtained by neglecting either of the sleptons.

\footnotetext{
${ }^{3}$ Similar conclusion is obtained in models which $\Delta a_{e, \mu}$ is explained by the loop corrections involving vectorlike leptons and a $Z^{\prime}$ boson [52-54].
} 
The slepton mass terms and interaction terms are given by

$$
\begin{aligned}
-\mathcal{L}_{F, \text { scal }}= & m_{\widetilde{L}}^{2} \widetilde{L}^{\dagger} \widetilde{L}+m_{\widetilde{E}}^{2} \widetilde{E}^{\dagger} \widetilde{E}+\left(A \widetilde{L}^{\dagger} H \widetilde{E}+\text { h.c. }\right) \\
& +\lambda_{L H}\left(\widetilde{L}^{\dagger} H\right)\left(H^{\dagger} \widetilde{L}\right)+\lambda_{L H}^{\prime}\left(\widetilde{L}^{\dagger} H\right)\left(\widetilde{L}^{\dagger} H\right) .
\end{aligned}
$$

Since the quartic couplings in eq. (2.8) are irrelevant to DM physics, we simply neglect these terms. The Yukawa couplings involving the DM are given by

$$
\mathcal{L}_{F, \text { Yukawa }}=\lambda_{L}^{i} \bar{\ell}_{L}^{i} \chi_{R} \widetilde{L}+\lambda_{R}^{i} \widetilde{E}^{\dagger} \bar{\chi}_{L} e_{R}^{i}+\text { h.c.. }
$$

For simplicity, we use the common notation, $\lambda_{L}^{i}$ and $\lambda_{R}^{i}$, for the portal Yukawa couplings in both scalar and fermion DM models. As in the scalar DM models, we assume that one of the portal Yukawa couplings is sizable in the minimal setup. The models with both couplings are discussed in section 5 .

If both $\widetilde{L}$ and $\widetilde{E}$ exist in the model, the trilinear terms induce the mixing between these states. The mass matrix is given by

$$
\left(\widetilde{E}^{\prime \dagger} \widetilde{E}^{\dagger}\right)\left(\begin{array}{cc}
m_{\widetilde{L}}^{2} & A v_{H} \\
A^{*} v_{H} & m_{\widetilde{E}}^{2}
\end{array}\right)\left(\begin{array}{c}
\widetilde{E}^{\prime} \\
\widetilde{E}
\end{array}\right),
$$

where $\widetilde{E}^{\prime}$ is the charged component of the doublet slepton $\widetilde{L}$. The mass eigenstates of the sleptons are defined as

$$
\left(\begin{array}{c}
\widetilde{E}^{\prime} \\
\widetilde{E}
\end{array}\right)=\left(\begin{array}{cc}
c_{\theta} & s_{\theta} \\
-s_{\theta} & c_{\theta}
\end{array}\right)\left(\begin{array}{c}
\tilde{E}_{1} \\
\tilde{E}_{2}
\end{array}\right),
$$

where $c_{\theta}, s_{\theta}$ satisfy $c_{\theta}^{2}+s_{\theta}^{2}=1$. Their masses are denoted as $m_{\widetilde{E}_{1}}$ and $m_{\widetilde{E}_{2}}$. The Yukawa couplings involving the DM and sleptons are given by

$$
\mathcal{L}_{F, \text { Yukawa }} \supset \bar{e}_{i}\left[\left(\lambda_{L}^{i} P_{R} c_{\theta}-\lambda_{R}^{i *} P_{L} s_{\theta}\right) \widetilde{E}_{1}+\left(\lambda_{L}^{i} P_{R} s_{\theta}+\lambda_{R}^{i *} P_{L} c_{\theta}\right) \widetilde{E}_{2}\right] \chi+\text { h.c.. }
$$

The new physics contribution to $\Delta a_{\mu}$ in the fermion DM model is given by [48-50]

$$
\begin{array}{r}
\Delta a_{\mu}=-\frac{m_{\mu}}{16 \pi^{2} m_{\chi}^{2}}\left[\left(c_{\theta}^{2}\left|\lambda_{L}^{\mu}\right|^{2}+s_{\theta}^{2}\left|\lambda_{R}^{\mu}\right|^{2}\right) m_{\mu} F_{s}\left(y_{1}\right)-c_{\theta} s_{\theta} \operatorname{Re}\left(\lambda_{L}^{\mu} \lambda_{R}^{\mu}\right) m_{\chi} G_{s}\left(y_{1}\right)\right. \\
\left.+\left(s_{\theta}^{2}\left|\lambda_{L}^{\mu}\right|^{2}+c_{\theta}^{2}\left|\lambda_{R}^{\mu}\right|^{2}\right) m_{\mu} F_{s}\left(y_{2}\right)+c_{\theta} s_{\theta} \operatorname{Re}\left(\lambda_{L}^{\mu} \lambda_{R}^{\mu}\right) m_{\chi} G_{s}\left(y_{2}\right)\right]
\end{array}
$$

where $y_{i}=m_{\chi}^{2} / m_{\tilde{E}_{i}}^{2}$. The loop functions are defined as

$$
F_{s}(x)=x \frac{1-6 x+3 x^{2}+2 x^{3}-6 x^{2} \log x}{6(1-x)^{4}}, \quad G_{s}(x)=x \frac{1-x^{2}+2 x \log x}{(1-x)^{3}} .
$$

As in the scalar DM models, both singlet and doublet sleptons are necessary to explain $\Delta a_{\mu}$ so that the chirality-flip effect proportional to $m_{\chi}$ is substantial.

Hereafter, when we analytically study generic features of the models, we will not assume any specific structure of the portal Yukawa couplings. The couplings are supposed 
to have arbitrary values and, therefore, be entirely flavor violating. If the DM has sizable couplings to two or more generations at the same time, such a setup will induce too large LFV processes to be consistent with experimental limits. In our numerical analysis, we will identify a structure of the portal couplings such that the DM couples exclusively to one of the three generations. The strong constraints from LFV processes are satisfied under this assumption. We will mainly focus on namely the muon-philic case, i.e. $\lambda_{L, R}^{\mu} \gg \lambda_{L, R}^{e}, \lambda_{L, R}^{\tau}$, motivated by the discrepancy in $\Delta a_{\mu}$.

\section{The LHC limits on extra lepton and slepton}

We discuss experimental limits from the LHC in this section. In the lepton portal DM models, the lightest mediator decays to a SM lepton and a DM particle. The pair production of the vectorlike leptons $E_{1}$ and sleptons $\widetilde{E}_{1}$ is

$$
p p \rightarrow E_{1} E_{1} \rightarrow e_{i} e_{i}+X X, \quad \text { and } \quad p p \rightarrow \widetilde{E}_{1} \widetilde{E}_{1} \rightarrow e_{i} e_{i}+\chi \chi,
$$

respectively. Both processes predict the signal with two SM leptons and a large missing energy in the final state which has been studied in slepton searches at the LHC [65-70]. We will study a case that the (s)lepton dominantly decays to a muon and a DM particle. The LHC limits in the electron-philic case will be similar to those in the muon-philic case, while the limits will be much weaker in the tau-philic case.

We estimate the experimental limits on the sleptons and the vectorlike leptons from the results in ref. [67]. To illustrate our method, we study a limit on the slepton first. The limit is estimated from the ATLAS analysis for doublet smuon $\widetilde{\mu}_{L}$ pair production. We define an efficiency $\epsilon_{\mathrm{SR}}$ in each signal region (SR) as

$$
\sigma_{\text {prod }}^{\text {ref }} \times \epsilon_{\mathrm{SR}}=\sigma_{\mathrm{SR}}^{\mathrm{exp}},
$$

where $\sigma_{\text {prod }}^{\text {ref }}$ is a cross section of $\widetilde{\mu}_{L} \widetilde{\mu}_{L}$ production and $\sigma_{\mathrm{SR}}^{\text {exp }}$ is an experimental upper bound on the effective cross section in the signal region. Here, $\widetilde{\mu}$ decays to a muon and a DM particle exclusively. The efficiency $\epsilon_{\mathrm{SR}}$ is a probability that pair-produced smuons pass the kinematic-cut of signal region.

We estimate the efficiency $\epsilon_{\mathrm{SR}}$ from the experimental limits as a function of the mass difference between slepton and DM, $\Delta m \equiv m_{\widetilde{E}_{1}}-m_{\chi}$. On the experimental bound in $\left(m_{\widetilde{E}_{1}}, m_{\chi}\right)$ plane, the efficiency is given by

$$
\epsilon_{\mathrm{SR}}(\Delta m) \sim \frac{\sigma_{\mathrm{SR}}^{\exp }}{\sigma_{\text {prod }}^{\text {ref }}\left(m_{\widetilde{E}_{1}}(\Delta m)\right)} .
$$

Here, the efficiency is assumed to be independent of the slepton mass approximately. The production cross section is, on the other hand, determined by the slepton mass. When the mass difference is larger than the limit on the slepton mass with massless DM, denoted by $m_{\widetilde{E}}^{\max }$, the efficiency is set at $\epsilon_{\mathrm{SR}}\left(m_{\widetilde{E}}^{\max }\right)$. By using this efficiency function of $\Delta m$, a point $\left(m_{\widetilde{E}_{1}}, m_{\chi}\right)$ is excluded if

$$
\sigma_{\text {prod }}\left(m_{\widetilde{E}_{1}}\right) \times \epsilon_{\mathrm{SR}}(\Delta m)>\sigma_{\mathrm{SR}}^{\exp }
$$



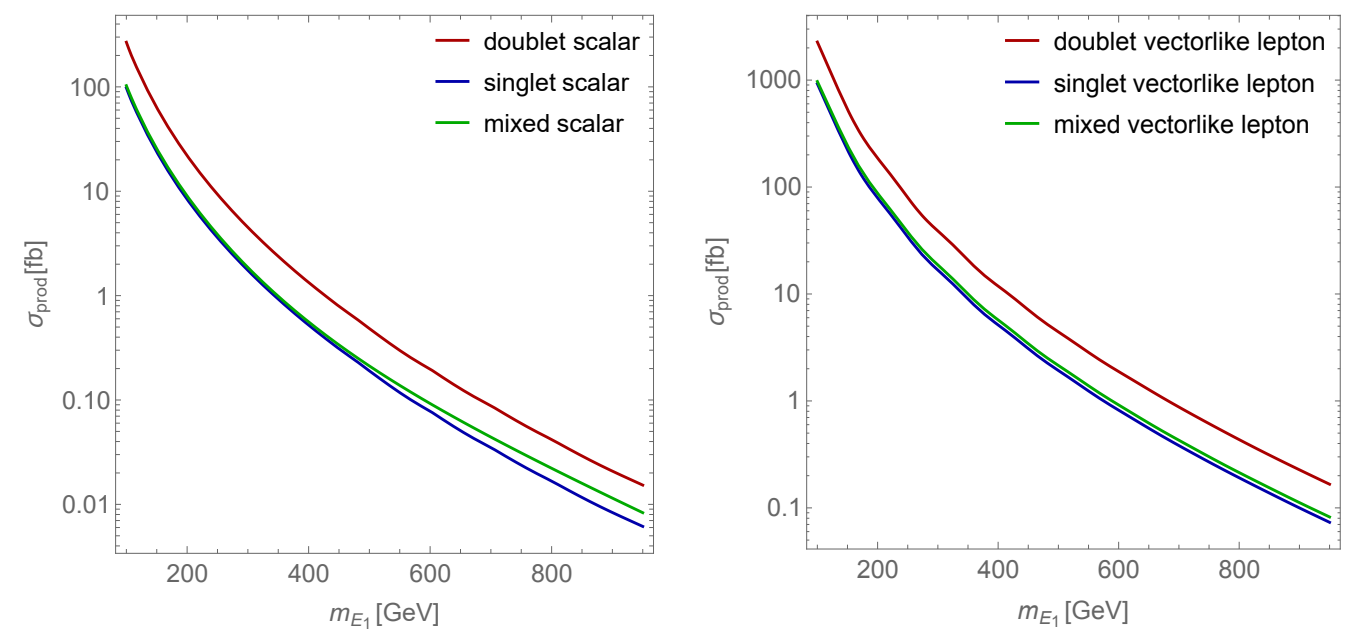

Figure 1. The pair production cross sections of the lightest sleptons (left) and the vectorlike leptons (right) with $\sqrt{s}=13 \mathrm{TeV}$.
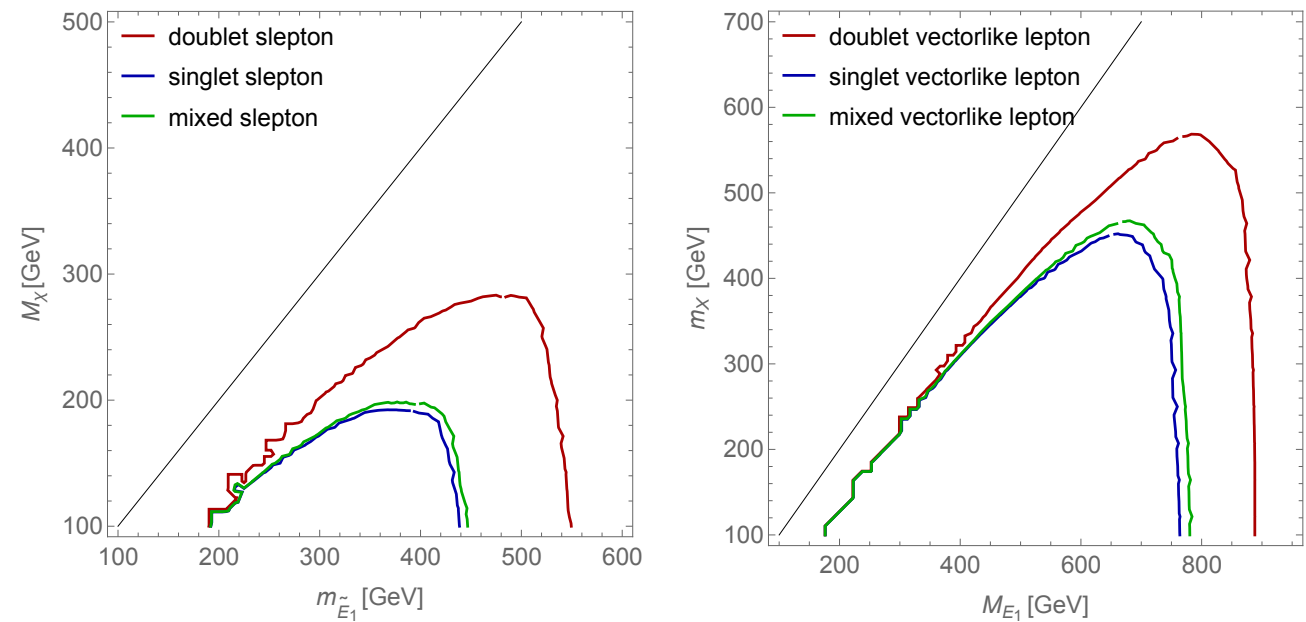

Figure 2. Experimental limits on the slepton (left) and vectorlike lepton (right) estimated from the ATLAS data [67].

in any signal region. In the analysis of ref. [67], there are two types of signal regions. One is designed for a mass spectrum with large mass difference and requires no jets in an event. The other is designed for a mass spectrum with small mass difference and requires a jet in an event. We refer to the signal regions without (with) jet for $\Delta m>200(\leq 200) \mathrm{GeV}$.

For the vectorlike lepton, we estimate a limit by analogy with the slepton search. We estimate the efficiency in eq. (3.3) from the limit on the degenerate slepton scenario, where the masses of left- and right-handed selectron and smuon are the same. Our estimations could be improved, using a limit that gives more similar shape as that for the vectorlike lepton.

Figure 1 shows production cross sections of a pair production of sleptons (left) and vectorlike leptons (right) with $\sqrt{s}=13 \mathrm{TeV}$. For the production cross sections of sleptons, we refer to the result of LHC SUSY Cross section Working Group [71-75]. The mixed slepton is defined as the lighter slepton when $s_{L}=s_{R}=s_{\theta}=1 / \sqrt{2}$. 
The production cross sections of the vectorlike leptons are calculated by MadGraph5_2_6_5 [76] based on a UFO [77] model file generated with FeynRules_2_3_32 [76, 78]. As in the vectorlike lepton case, the mixed extra lepton is defined as the lighter one when $s_{L}=s_{R}=s_{\theta}=1 / \sqrt{2}$.

Figure 2 shows the estimated 95\% C.L. upper limits on the mediator lepton and DM masses. The red, blue and green lines show the limit for the doublet, singlet and maximally mixed sleptons (vectorlike leptons), respectively. The doublet leptons are more constrained than the others because of the larger production cross sections. The limit on singlet slepton shows good agreement with the experimental limit in ref. [67].

The analysis in ref. [67] excludes parameter space where the mass difference is larger than about $100 \mathrm{GeV}$. More degenerate region would be excluded by more dedicated searches exploiting an initial state radiation and soft leptons [66, 79]. The limits, however, exist only in restricted parameter space where the mass difference is about $10 \mathrm{GeV}$ and the tightest limit is about $250 \mathrm{GeV}$ for the degenerate four sleptons scenario. The limits from these searches are not shown in the following analyses, but we shall note that a shallow parameter space with $\Delta m \lesssim 10 \mathrm{GeV}$ would be constrained by these searches.

Let us comment on the heavier mediator leptons. The heavier states $E_{2}$ and $\widetilde{E}_{2}$ can decay to a SM lepton and a DM particle as the lighter ones can. In addition, these may decay to a lighter mediator lepton and a SM boson. For instance, $E_{2} \rightarrow E_{1} Z \rightarrow \mu Z X$ is possible and can give clean signals with three charged leptons and large missing energy per vectorlike lepton $E_{2}$. The branching fractions to a SM boson can be comparable with or even dominate over that to a lepton and a DM particle depending on the couplings and the mass spectrum. This is an interesting possibility to discover the lepton portal DM models but this is beyond the scope of this paper. In the following, we only show the limits from a pair production of the lightest mediator leptons in figure 2 .

\section{Dark matter physics in the minimal models}

In this section, we study DM physics and discuss constraints on the models with the minimal matter contents, where either an $\mathrm{SU}(2)_{L}$ doublet or singlet mediator field exists. There are four types of DM fields (real/complex/Dirac/Majorana), and the mediator field is a doublet $L / \widetilde{L}$ (case (i)) or a singlet $E / \widetilde{E}$ (case (ii)) under $\mathrm{SU}(2)_{L}$. The next-to-minimal models with both mediator fields will be discussed in the next section.

\subsection{Annihilation cross sections}

The pair annihilation is a basic property of particle DM. It governs the DM abundance based on the thermal freeze-out mechanism. If annihilation occurs in halo, it also contributes to cosmic ray flux which can be probed by indirect searches at telescopes. Since DM particles are non-relativistic at the freeze-out temperature and also in halo, it is useful to expand the pair annihilation cross section in terms of relative velocity $v$ of DM particles:

$$
(\sigma v)_{A}=a_{A}+b_{A} v^{2}+c_{A} v^{4}+\mathcal{O}\left(v^{6}\right)
$$



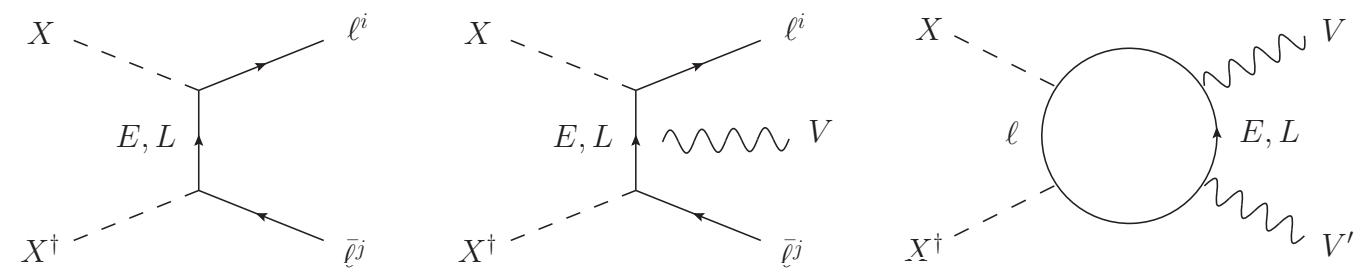

Figure 3. Example diagrams of the DM pair annihilation in the scalar DM model. In the fermion DM model, the dashed lines for $X$ and solid lines for $E$ and $L$ are replaced by the solid lines for $\chi$ and dashed lines for $\widetilde{E}$ and $\widetilde{L}$, respectively.

where $a_{A}, b_{A}$ and $c_{A}$ are dubbed as partial $s$-wave, $p$-wave and $d$-wave contributions, respectively. Here, the subscript $A$ represents a final state of the annihilation process. In the lepton portal DM models, the Yukawa couplings in eqs. (2.2) and (2.9) induce various annihilation processes. In this section, we summarize the important features of the DM annihilation to $\ell_{i} \bar{\ell}_{j}, \ell_{i} \bar{\ell}_{j} V$ and $V V^{\prime}$, where $\ell_{i}$ is a SM charged lepton or neutrino and $V^{(\prime)}$ is a SM gauge boson. Here, $i=1,2,3$ is the flavor index. Note that the neutrinos have only left-handed component. The sample diagrams are shown in figure 3. The full analytical formulas are shown in appendix A.

Figure 4 shows ratios of thermal averaged cross sections, $\langle\sigma v\rangle_{\ell \bar{\ell} V}$ and $\langle\sigma v\rangle_{V V^{\prime}}$, to that of the tree-level two-body annihilations $\langle\sigma v\rangle_{\ell \bar{\ell}}$, with $m_{\mathrm{DM}}=500 \mathrm{GeV}$, the $\mathrm{SU}(2)_{L}$ doublet mediators and the muon-philic coupling $\left(\lambda_{L}^{\mu} \neq 0, \lambda_{L}^{e}=\lambda_{L}^{\tau}=0\right)$. Here, $\langle\sigma v\rangle_{\bar{\ell}(V)}=$ $\langle\sigma v\rangle_{\mu \bar{\mu}(V)}+\langle\sigma v\rangle_{\nu_{\mu} \bar{\nu}_{\mu}(V)}$ and $\langle\sigma v\rangle_{\ell \bar{\ell} W}=\langle\sigma v\rangle_{\mu \bar{\nu}_{\mu} W^{+}}+\langle\sigma v\rangle_{\bar{\mu}_{\mu} W^{-}}$. The velocity suppressed processes are evaluated at the freeze-out temperature: $\left\langle v^{2}\right\rangle \simeq 0.24$ and $\left\langle v^{4}\right\rangle \simeq 0.1$. The plots are independent of the coupling $\lambda_{L}^{\mu}$, since it cancels out between the numerator and denominator. In the real, complex and Majorana cases, these are also independent of the choice of the lepton flavor as far as only one of the couplings is sizable. For the Dirac DM, however, there is a slight flavor dependence in $\langle\sigma v\rangle_{\ell \bar{\ell} \gamma}$ due to a collinear divergence in the limit $m_{\ell} \rightarrow 0$. This will be discussed later more concretely. Phenomenologically important effects in these processes are summarized in the following.

DM pair annihilation into $\ell_{i} \bar{\ell}_{j}$. Figure 3 (left) shows the DM pair annihilation into a pair of SM leptons $\ell_{i} \bar{\ell}_{j}$, where $\ell_{i}=e_{i}, \nu_{i}$. In the case (i) (weak doublet mediator), the $s$-wave contributions are given by

$$
a_{\ell_{i} \ell_{j}}=\frac{\left|\lambda_{L}^{i} \lambda_{L}^{j}\right|^{2}}{32 \pi m_{\mathrm{DM}}^{2}\left(1+r^{2}\right)^{2}} \times \begin{cases}4\left(\epsilon_{i}+\epsilon_{j}\right) & \text { real scalar } \\ \epsilon_{i}+\epsilon_{j} & \text { complex scalar } \\ \left(\epsilon_{i}+\epsilon_{j}\right) / 2 & \text { Majorana fermion } \\ 1 & \text { Dirac fermion }\end{cases}
$$

where $m_{\mathrm{DM}}$ denotes the DM mass: $m_{\mathrm{DM}}$ is identical to $m_{X}$ in the scalar DM model and $m_{\chi}$ in the fermion DM model. The ratios $r$ and $\epsilon_{i}$ are defined as $r \equiv m_{L} / m_{X}$ and $\epsilon_{i} \equiv m_{i}^{2} / m_{X}^{2}$ in the scalar DM model, and $r \equiv m_{\widetilde{L}} / m_{\chi}$ and $\epsilon_{i} \equiv m_{i}^{2} / m_{\chi}^{2}$ in the fermion DM model. Here, $m_{i}$ is the mass of the lepton $\ell_{i}$. The sub-leading order effects in $\epsilon_{i}$ are neglected. In 

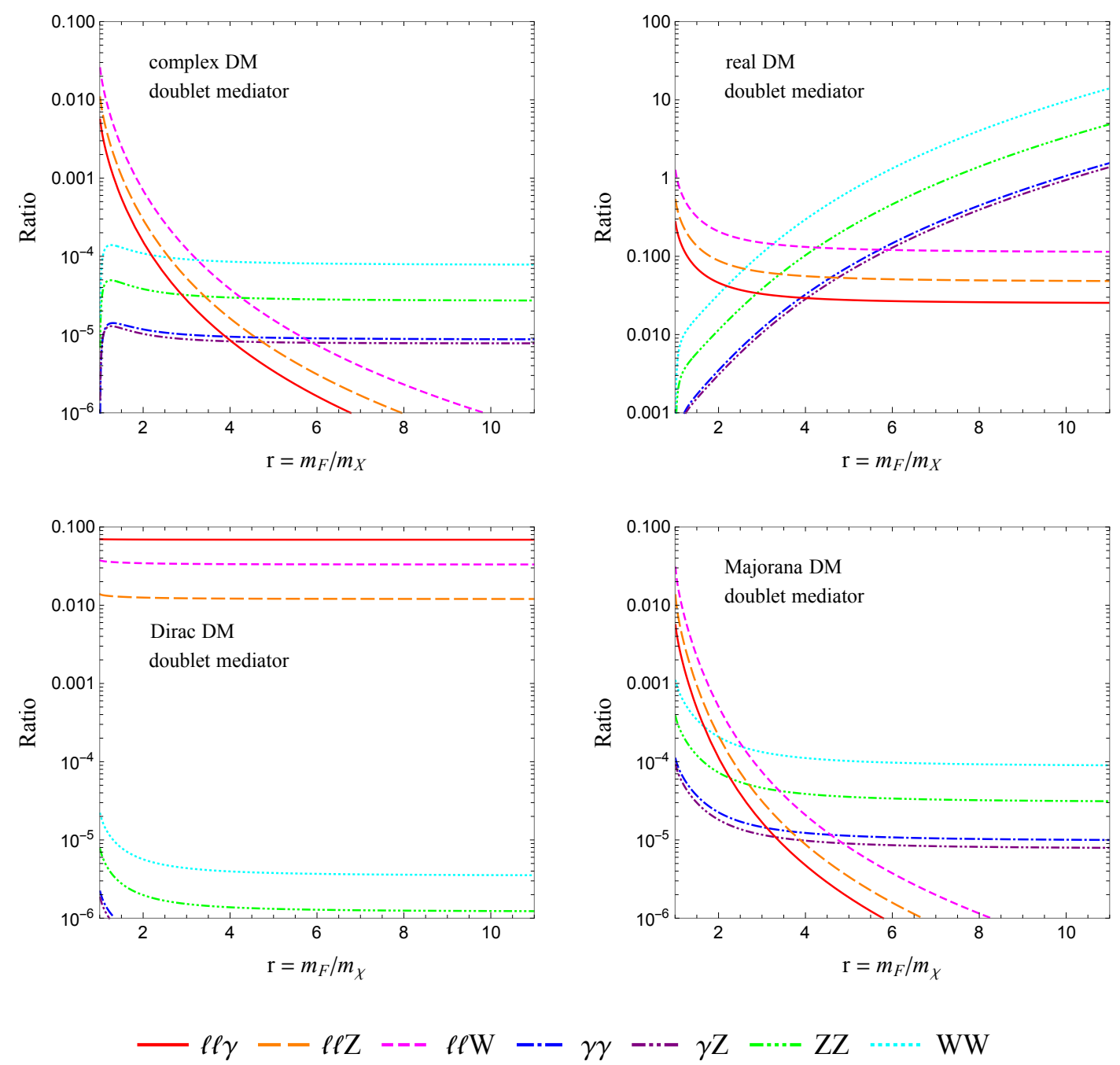

Figure 4. Ratio of cross section $\langle\sigma v\rangle_{A} /\langle\sigma v\rangle_{\ell \bar{\ell}}$ in various annihilation channels as a function of $r$, in the complex (top-left), real (top-right), Dirac (bottom-left) and Majorana (bottom-right) DM models. We assume the $\mathrm{SU}(2)_{L}$ doublet mediators and the muon-philic coupling. The DM mass is fixed at $500 \mathrm{GeV}$ in all cases and DM velocity is set to the thermal averaged values at the freeze-out: $\left\langle v^{2}\right\rangle \simeq 0.24$ and $\left\langle v^{4}\right\rangle \simeq 0.1$. The processes associated with $W$ boson are absent in the models with the $\mathrm{SU}(2)_{L}$ singlet mediator.

the models except for the Dirac DM model, the $s$-wave contribution is helicity suppressed $\left(\epsilon_{i} \ll 1\right)$. In the real DM, the $p$-wave contribution $b_{\ell_{i} \bar{\ell}_{j}}$ is also helicity suppressed and the leading contribution is the $d$-wave. Thus the annihilation cross section is considerably suppressed and other processes discussed below are relatively important. The expressions for the case (ii) is obtained by replacing $\lambda_{L}^{i} \rightarrow \lambda_{R}^{i *}, m_{L} \rightarrow m_{E}$ and $m_{\widetilde{L}} \rightarrow m_{\widetilde{E}}$. The full analytical expressions of the expansion coefficients are given in appendix A.

DM pair annihilation into $\ell_{i} \bar{\ell}_{j} \boldsymbol{V}$. Figure 3 (middle) shows a diagram for the DM annihilation into a pair of leptons, accompanied with a gauge boson $V$, where $V=\gamma, Z, W$. 
In all types of DM, these processes have $s$-wave contributions without helicity suppression, while these are suppressed by a gauge coupling and three-body phase space. Using the parametrization of eq. (4.1), the relative importance at the freeze-out is evaluated by

$$
\frac{\langle\sigma v\rangle_{\ell \bar{\ell} V}}{\langle\sigma\rangle_{\ell \bar{\ell}}} \sim \frac{\alpha / \pi}{\left\langle v^{n}\right\rangle}
$$

where $n(=0,2,4)$ is a power of $v$ of the dominant two-body annihilation cross section $\langle\sigma v\rangle_{\ell \bar{\ell}}$ in each model. As shown in figure 4, this ratio is $\mathcal{O}(1-0.1)$ in the real DM model $(n=4)$ depending on $r$, while no more than 0.1 in the other three models. We will therefore include these processes in calculating the DM thermal abundance in the real DM model. The concrete expressions of the cross sections as well as their squared amplitudes are listed in appendix A.

In general, the three-body processes are superposition of the final state radiation (FSR) from on-shell leptons, and an emission from the off-shell intermediate state, namely the virtual internal bremsstrahlung (VIB). The differential cross section of the FSR is related to the two-body cross section [80],

$$
\frac{d(\sigma v)_{\ell \bar{\ell} \gamma}^{\mathrm{FSR}}}{d x} \approx(\sigma v)_{\bar{\ell} \bar{\ell}} \frac{Q_{\ell}^{2} \alpha}{\pi} \frac{(1-x)^{2}+1}{x} \log \left(\frac{4 m_{\mathrm{DM}}^{2}(1-x)}{m_{\ell}^{2}}\right),
$$

independently of the DM types. Here, $x$ is defined as $x=2 E_{\gamma} / \sqrt{s}$ with the photon energy, $E_{\gamma}$. If $(\sigma v)_{\ell \bar{\ell}}$ is helicity suppressed, the FSR contribution is also suppressed and negligible. The real, complex and Majorana DM models meet this condition. In these models, the three-body processes are dominated by the VIB, and exhibit a sharp spectrum of emitted vector bosons $V$ around $E_{V}=m_{X(\chi)}$ if the DM and mediator masses are nearly degenerate [81-86]. Figure 5 shows the photon spectrum from the three-body annihilation $\chi \chi \rightarrow \ell_{i} \bar{\ell}_{j} \gamma$ in the Majorana DM model. The spectrum in the scalar DM models is the same as this. The photon spectrum in the VIB process fairly looks like a monochromatic spectrum within detector resolution. This sharp spectrum will be a distinctive signal of these DM models.

In the Dirac model, $(\sigma v)_{\ell \bar{\ell}}$ is not helicity suppressed, so that the FSR entirely dominates the three-body process. It follows from eq. (4.4) that the FSR cross section is not welldefined in the massless lepton limit, so that we have to keep the lepton mass finite to evaluate it. This leads to the slight flavor dependence of $\langle\sigma v\rangle_{\ell \bar{\ell} \gamma} /\langle\sigma v\rangle_{\ell \bar{\ell}}$, as mentioned above. Moreover, when we integrate over $x$, we encounter a divergence at the infrared edge $x=0$. This can be eliminated by taking radiative corrections to the two-body processes into account. In figure 4 (bottom-left), to evaluate the size of the three-body process, we just regularized the infrared divergence by integrating over the range $0.1<x<1$. This corresponds to introducing a sharp infrared cutoff at $E_{\gamma}=0.1 m_{\mathrm{DM}}$. We have confirmed that the numerical result obtained in this way is smaller than the so-called double logarithm approximation, $(\sigma v)_{\ell \bar{\ell}}(\alpha / \pi) \log \left(4 m_{\mathrm{DM}}^{2} / m_{\ell}^{2}\right) \log \left(m_{\mathrm{DM}}^{2} / \Lambda_{I R}^{2}\right)$, by a factor $\sim 1.5$.

DM pair annihilation into $\boldsymbol{V} \boldsymbol{V}^{\prime}$. The DM can pair-annihilate into two gauge bosons via loop diagrams shown in the figure 3 (right). The possible final states are $V V^{\prime}=$ 


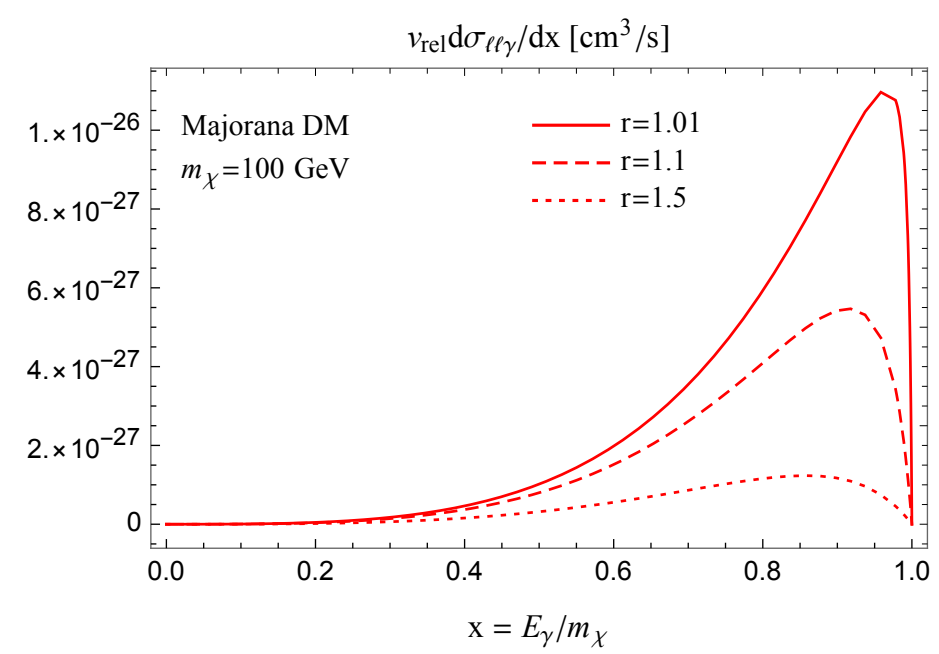

Figure 5. Photon spectrum from $\chi \bar{\chi} \rightarrow \ell \bar{\ell} \gamma$ in the Majorana DM model. Here, the DM mass is $m_{\chi}=100 \mathrm{GeV}$ and $r=m_{\tilde{L}} / m_{\chi}$ and the portal Yukawa coupling is unity. The spectrum is completely the same for the scalar DM.

$\gamma \gamma, \gamma Z, Z Z, W^{+} W^{-}$. We do not consider the annihilation into $h Z, h h$, since these are further suppressed by the small Higgs Yukawa couplings of the charged leptons. These processes cannot be leading contributions at the freeze-out in every setup due to the suppression via the gauge couplings and the loop factor.

Nevertheless, $X X \rightarrow \gamma \gamma, \gamma Z$ will be significant at indirect detection of the real scalar DM. For large $r=m_{E_{1}} / m_{X}$, the cross section scales as

$$
(\sigma v)_{V V^{\prime}} \propto \frac{1}{r^{4}}, \quad \text { while } \quad(\sigma v)_{\ell \bar{\ell}(V)} \propto \frac{1}{r^{8}} .
$$

Hence, the loop annihilation $X X \rightarrow \gamma \gamma, \gamma Z$ can be a sizable fraction of the total annihilation cross section for large $r$. For example, $(\sigma v)_{\gamma \gamma} /(\sigma v)_{\bar{\ell} \bar{\ell}} \sim 0.01$ is realized for $r=3$. This small fraction is irrelevant to the DM abundance, but it can be crucial in gamma-ray searches since produced photons in $X X \rightarrow \gamma \gamma, \gamma Z$ are monochromatic and may provide distinctive signals.

\subsection{Relic density}

In the thermal freeze-out scenario, it is assumed that the DM abundance was produced in the thermal bath. The produced number density is calculated by solving the Boltzmann equation,

$$
\frac{d n_{\mathrm{DM}}}{d t}+3 H n_{\mathrm{DM}}=-\langle\sigma v\rangle_{\mathrm{eff}}\left[n_{\mathrm{DM}}^{2}-\left(n_{\mathrm{DM}}^{\mathrm{eq}}\right)^{2}\right]
$$

where $H$ is the Hubble rate and $n_{\mathrm{DM}}\left(n_{\mathrm{DM}}^{\mathrm{eq}}\right)$ is the (equilibrium) number density of DM. Here, $\langle\sigma v\rangle_{\text {eff }}$ is the effective annihilation cross section of DM, and is expressed in terms of the DM pair annihilation and coannihilation. As a concrete example, in the real scalar DM model, it is approximately given by,

$$
\langle\sigma v\rangle_{\mathrm{eff}} \simeq\langle\sigma v\rangle+2\left(\left\langle\sigma_{X L} v\right\rangle+\left\langle\sigma_{X} \bar{L} v\right\rangle\right) \frac{g_{L} m_{L}^{3 / 2}}{g_{X} m_{X}^{3 / 2}} e^{-\Delta m / T}+2\left\langle\sigma_{L \bar{L}} v\right\rangle \frac{g_{L}^{2} m_{L}^{3}}{g_{X}^{2} m_{X}^{3}} e^{-2 \Delta m / T},
$$


in the non-relativistic regime $\left(T \ll m_{X}\right)$. Here, we define $\Delta m=m_{L}-m_{X}$ and $g_{X}\left(g_{L}\right)$ is the internal degrees of freedom for $X(L) .^{4}\langle\sigma v\rangle=\langle\sigma v\rangle_{\bar{\ell} \bar{\ell}}+\langle\sigma v\rangle_{\ell \bar{\ell} V}+\langle\sigma v\rangle_{V V^{\prime}}$ represents the thermal average of the total DM pair annihilation cross section given in the previous section, while $\left\langle\sigma_{X L(X \bar{L})} v\right\rangle$ and $\left\langle\sigma_{L \bar{L}} v\right\rangle$ are those of coannihilation cross sections whose initial states are $X L(X \bar{L})$ and $L \bar{L}$, respectively. The effective cross section $\langle\sigma v\rangle_{\text {eff }}$ at the freeze-out temperature $T_{f} \simeq m_{\mathrm{DM}} / 20$, when the DM number density in a comoving volume is almost fixed, is crucial for the thermal density. It follows from eq. (4.7) that the coannihilation processes are important when the exponential suppressions are not strong. Naive estimate suggests that the coannihilation is effective if the exponential factor,

$$
e^{-\frac{\Delta m}{T_{f}}}=e^{-\frac{m_{X}}{T} \frac{\Delta m}{m_{X}}} \simeq e^{-20 \frac{\Delta m}{m_{X}}}
$$

is not too small, i.e. $\Delta m / m_{X} \lesssim \mathcal{O}(0.1)$ is satisfied. Indeed, as we will see in section 4.5 , the coannihilation processes help to deplete the DM thermal abundance down to the observed value with a smaller Yukawa coupling in nearly mass degenerate region. In our analysis, we employ micromegas_4.3.5 [89] to numerically solve the Boltzmann equation together with all coannihilation processes. In the real scalar DM model, we also consider $s$-wave contributions of the three-body and loop induced processes. These higher-order processes are no more than $10 \%$ of the leading processes in the other DM models as shown in figure 4 , and therefore are neglected.

\subsection{Indirect detection}

The indirect detection experiments look for cosmic ray fluxes, such as gamma ray, antiproton, positron and neutrino, originated from DM annihilation on top of astrophysical backgrounds. These are good complementary tools of direct detections to probe DM, although there are large systematic uncertainties of astrophysical contributions. In this paper, we will not perform any new data analysis, and will simply rescale the sensitivity curves derived in the literature. Here, we shall review the constraints from the indirect searches for the lepton portal DM models. We will see that the DM annihilation to $\bar{\ell} \bar{\ell}$ or $\bar{\ell} \gamma$ will provide signals to constrain the models. We will discuss only the real scalar, Majorana and Dirac fermion DM. The constraints on the complex scalar DM will be very similar to those on the Majorana DM since the squared amplitudes of the relevant processes are the same. ${ }^{5}$

The most prominent target in cosmic ray searches for DM annihilation is a spectral feature, such as gamma line or VIB photon. Such a sharp spectrum can be well disentangled from uncertain astrophysical backgrounds, since it is difficult to attribute the sharp photon spectrum to one astrophysical process. The search for the spectral feature is often a unique way to observe DM signals from the sky, particularly when the tree-level two-body annihilation is suppressed.

The spectral features in the lepton portal DM models have been examined in the literature. It has been found that the sensitivity can considerably be improved when we

\footnotetext{
${ }^{4}$ For the exact expression of the effective cross section, see e.g. [87, 88].

${ }^{5}$ See appendix A for the explicit forms.
} 
exploit the photon spectrum instead of the continuum photon flux. In the Majorana DM, for example, dedicated searches for the spectral features set orders of magnitude stronger upper bounds on $\langle\sigma v\rangle_{\ell \bar{\ell} \gamma}$ than the bounds from the continuum gamma-ray observation of dwarf spheroidal (dSph) galaxies [81]. The study of the spectral feature is then extended to the pair annihilation into $\gamma \gamma$, showing the upper limits on the combined annihilation cross section: $\langle\sigma v\rangle_{\ell \bar{\ell} \gamma}+2\langle\sigma v\rangle_{\gamma \gamma} \lesssim 10^{-26}-10^{-27} \mathrm{~cm}^{3} / \mathrm{s}$ for the DM mass ranging from $40 \mathrm{GeV}$ to $10 \mathrm{TeV}$ [82]. Note that EW gauge invariance requires the existence of weak VIB processes, such as $\ell \bar{\ell} Z$. These exhibit a spectral feature of an anti-proton flux from decays and hadronization of the final state weak bosons, similarly to that of photon flux. The impact of the weak VIB emission on the PAMELA anti-proton search is studied in the Majorana DM model in refs. [90, 91]. Unfortunately, these cosmic-ray signals of the Majorana DM cannot be observed even at future telescopes unless an astrophysical boost factor is larger than $\mathcal{O}(10)$.

The search for the spectral features can impose good complementary bounds on the real scalar DM. As will be discussed in section 4.4, this type of DM is almost free from the direct detection bound. On the other hand, the fraction of the annihilation into $\ell \bar{\ell} \gamma$ and $\gamma \gamma$ in the total annihilation is sizable because of the strong suppression in the two-body process $X X \rightarrow \ell \bar{\ell}$. This suggests that more fluxes are predicted than those in the Majorana DM, opening up a possibility to discover DM signals in gamma-ray spectrum. It is shown in ref. [86] that the future GAMMA-400 [92] and CTA [93] experiments can probe the real scalar DM where $m_{X} \geq 100 \mathrm{GeV}$ and $m_{E_{1}} / m_{X} \geq 1.2$. The sensitivity prospect will be shown in figure 7.

For the Dirac DM, the spectral feature is absent, since the gamma-ray flux is dominated by the smooth FSR and continuum secondary gamma-ray. The latter originates from the decay and fragmentation of the SM particles produced by the DM annihilation. The gamma-ray observation of dSph galaxies therefore has the best sensitivity. The strongest limit is set on the tau-philic DM. In that case, the lower limit on $\langle\sigma v\rangle_{\tau \bar{\tau}}$ lies below the canonical thermal relic cross section $\sigma_{0} \sim 3 \times 10^{-26}\left[\mathrm{~cm}^{3} / \mathrm{s}\right]$ for $m_{\chi} \lesssim 100 \mathrm{GeV}$. In the muon-philic or electron-philic DM models with $\langle\sigma v\rangle_{\mu \bar{\mu}, e \bar{e}}=\sigma_{0}$, the lower bound on the DM mass is around $10 \mathrm{GeV}$ [94]. These bounds can be simply applied to the Dirac DM, since the unsuppressed two-body process $\chi \bar{\chi} \rightarrow \ell \bar{\ell}$ is mainly responsible for the thermal freeze-out. In the other DM types, the limit from the observation of dSph galaxies is much weaker than those from the gamma line observations [82].

We briefly comment on other possible constraints. Of particular importance is constraints from positron flux observations. In [95, 96], upper limits on annihilation cross section into leptonic final states are derived based on the AMS-02 data of the positron fraction [97]. The most stringent limit is posed on the $e^{+} e^{-}$channel, since the positron spectrum is so sharp even after propagation in galactic space that the spectral search is applicable. The $95 \%$ C.L. lower limit on the DM mass is $100 \mathrm{GeV}$, when the thermal relic annihilation cross section into $e^{+} e^{-}$is assumed [96]. The constraint on the $\mu^{+} \mu^{-}$and $\tau^{+} \tau^{-}$channels (or associated VIB processes) is much weaker, since the positron spectrum is broader and it becomes difficult to disentangle the DM signal from the smooth astrophysical background. For further detail of the analysis, see refs. [95, 96]. In the lepton 

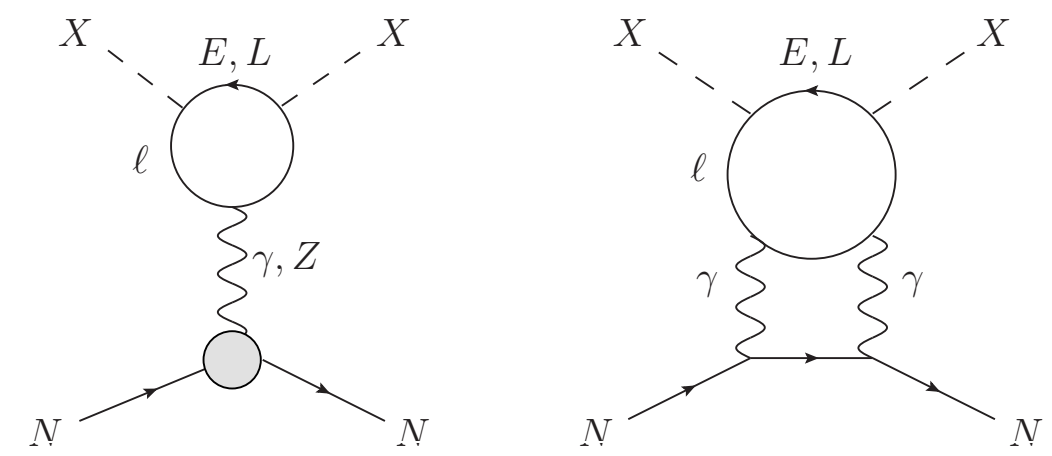

Figure 6. Example diagrams for DM-nucleon scattering at direct detection: (left) photon and $Z$ penguin contributions for the complex DM; (right) leading 2-loop contribution for the real DM. There are similar penguin contributions in the fermion DM.

portal DM models, the positron bound is relevant only to the Dirac DM and gives the limit on $m_{X} \lesssim 100 \mathrm{GeV}$ for the $e^{+} e^{-}$channel. Note that the other three types predict a sharp positron spectrum in the $e \bar{e} \gamma$ process, but the cross section is nevertheless too small to bring the positron constraints into play.

The lepton portal DM models predict neutrino fluxes as well. If the leptonic mediator is a weak doublet, the cross section can be sizable since the tree-level annihilation into $\nu \bar{\nu}$ is possible. The produced flux can be detected at neutrino telescopes. So far, the observations at neutrino telescopes have found no significant excess of neutrinos over the background. This is interpreted as an upper bound on the annihilation cross section. For instance, the ANTARES neutrino telescope has searched for self-annihilation of DM in the center of the Milky Way, and reported bounds on the five representative annihilation channels $[98,99]$. Of these, $\tau^{+} \tau^{-}, \mu^{+} \mu^{-}$, and $\nu \bar{\nu}$ are relevant to the lepton portal DM models. Using the latest 11 years data, and assuming the NFW halo profile and $100 \%$ branching ratio, the upper limit on $\langle\sigma v\rangle_{\nu \bar{\nu}}$ is $10^{-23}-10^{-24}\left[\mathrm{~cm}^{3} / \mathrm{s}\right]$ for the DM mass ranging from $50 \mathrm{GeV}$ to $100 \mathrm{TeV}$ [99]. The limits on the $\tau^{+} \tau^{-}$and $\mu^{+} \mu^{-}$channels are weaker than the former under the same assumption. The searches at IceCube neutrino observatory set similar upper bounds on them [100]. In our models, $\langle\sigma v\rangle_{\nu \bar{\nu}}$ is less than $10^{-26}\left[\mathrm{~cm}^{3} / \mathrm{s}\right]$ in every setup, so that the constraint from the neutrino flux has no impact on the models.

\subsection{Direct detection}

In the lepton portal DM models, there is no tree-level scattering between the DM and a nucleus. As is well known, primary contribution is from photon exchanging at loop levels in all types of the DM models. The diagrams are shown in figure 6 . The relevant DM-photon effective interaction depends on the DM types and masses. There are also similar $Z$ exchanging contributions in which photons in the leading diagrams are replaced by $Z$ bosons. These are, however, suppressed by lepton masses, so that these become sub-leading. In the following, we will summarize typical features of the leading process in each model. See appendix A for the full analytical expressions for the photon and $Z$ contributions. 
In the complex scalar DM model, the photon penguin diagram shown in figure 6 (left) is the leading contribution. The induced DM-nucleon effective operator is given by

$$
\mathcal{L}_{\text {eff }}^{S} \supset C_{V, N}\left(i X^{\dagger} \overleftrightarrow{\partial_{\mu}} X\right)\left(\bar{N} \gamma^{\mu} N\right)
$$

where $N=p, n$. Here, we define $\phi_{2} \overleftrightarrow{\partial_{\mu}} \phi_{1} \equiv \phi_{2}\left(\partial_{\mu} \phi_{1}\right)-\left(\partial_{\mu} \phi_{2}\right) \phi_{1}$. In the limit of $m_{L} \gg$ $m_{i}, m_{X}$, the coefficient $C_{V, N}$ is dominantly given by the photon-penguin diagram, $C_{V, N}^{\gamma}$,

$$
C_{V, N}^{\gamma} \simeq \frac{\alpha Q_{f} Q_{N}}{12 \pi m_{L}^{2}} \sum_{i}\left|\lambda_{L}^{i}\right|^{2}\left(\frac{3}{2}+\log \frac{m_{i}^{2}}{m_{L}^{2}}\right)
$$

in the case (i). The expression for the case (ii) is obtained by formally replacing $\lambda_{L}^{i} \rightarrow \lambda_{R}^{i *}$ and $m_{L} \rightarrow m_{E}$. When the vectorlike lepton is heavy, the cross section can be enhanced by the logarithmic term, leading to strong limits from direct detection experiments.

In the real scalar DM model, there is no penguin-type contribution. The leading contribution arises at two-loop level via two photon-exchanging [101]. The diagram is shown in figure 6 (right). The DM-nucleon scattering cross section is so suppressed that there are no significant constraints from the direct detection. When DM couples to the electron, DM-electron scatterings are induced at tree level. This scattering is relevant only in light mass region, typically $m_{X} \lesssim 1 \mathrm{GeV}$, while in this paper we focus on heavier DM masses, $m_{X} \gtrsim 100 \mathrm{GeV}$, since lighter DM scenario would be strictly constrained by, e.g. the LEP experiment. See refs. [102-105], for recent studies of limits on DM-electron scattering.

In the fermion DM models, there are multi-pole interactions,

$$
\mathcal{L}_{\text {multipole }}^{F}=b_{\chi} \bar{\chi} \gamma^{\mu} \chi \partial^{\nu} F_{\mu \nu}+\frac{\mu_{\chi}}{2} \bar{\chi} \sigma^{\mu \nu} \chi F_{\mu \nu}+a_{\chi} \bar{\chi} \gamma^{\mu} \gamma^{5} \chi \partial^{\nu} F_{\mu \nu}+i \frac{d_{\chi}}{2} \bar{\chi} \sigma^{\mu \nu} \gamma^{5} \chi F_{\mu \nu},
$$

in addition to the contact-type DM-nucleon effective interactions:

$$
\mathcal{L}_{\text {eff }}^{F}=\sum_{N=p, n}\left(C_{S, N} \bar{\chi} \chi \bar{N} N+C_{V, N} \bar{\chi} \gamma^{\mu} \chi \bar{N} \gamma_{\mu} N\right) .
$$

The differential cross section for elastic DM-nucleus scattering is expressed in terms of the Wilson coefficients:

$$
\begin{aligned}
\frac{d \sigma}{d E_{R}}= & \frac{\alpha Z^{2}}{v^{2}}\left[\mu_{\chi}^{2}\left(\frac{v^{2}}{E_{R}}-\frac{m_{N}}{2 \mu_{\mathrm{red}}^{2}}\right)+d_{\chi}^{2}\left(\frac{1}{E_{R}}-\frac{1}{m_{\chi}}\right)\right]\left|F\left(E_{R}\right)\right|^{2}+\frac{m_{N} f_{A}^{2}}{2 \pi v^{2}}\left|F\left(E_{R}\right)\right|^{2} \\
& +\frac{\alpha Z^{2}}{v^{2}} a_{\chi}^{2}\left[2 m_{N} v^{2}-\frac{\left(m_{N}+m_{\chi}\right)^{2}}{m_{\chi}^{2}} E_{R}\right]\left|F\left(E_{R}\right)\right|^{2} \\
& +\frac{m_{N} \mu_{A}^{2}}{2 \pi v^{2}}\left(2 \mu_{\chi}^{2}+d_{\chi}^{2} v^{2}+4 m_{N} E_{R} a_{\chi}^{2}\right) \frac{J_{A}+1}{3 J_{A}}\left|F_{\text {spin }}\left(E_{R}\right)\right|^{2}
\end{aligned}
$$

Note that this is calculated in the Dirac DM case. Here, $\mu_{\text {red }}=m_{\chi} m_{N} /\left(m_{\chi}+m_{N}\right)$ is the reduced mass of the DM and a nucleus. $f_{A}=Z\left(C_{S, p}+C_{V, p}-e b_{\chi}-e \mu_{\chi} /\left(2 m_{\chi}\right)\right)+$ $(A-Z)\left(C_{S, n}+C_{V, n}\right)$, where $Z$ and $A$ is an atomic number and weight of a nucleus, and $m_{N}, J_{A}$ and $\mu_{A}$ are the nuclear mass, spin and magnetic moment, respectively. In our models, $C_{S, N}$ and $C_{V, N}$ are induced by the $Z$ boson and Higgs penguin diagrams. We use 
the Helm form factor normalized with $F(0)=1$ for $F\left(E_{R}\right)$, and use a spin form factor with thin-shell approximation derived in [106] for $F_{\text {spin }}\left(E_{R}\right)$. The first two lines are the spin-independent contributions and the third line is the spin-dependent one. For the spinindependent one, there are non-contact contributions which appear with $1 / E_{R}$ and lead to infrared enhancement, while these are absent in the spin-dependent part. It should be noted that due to the non-contact contributions, we cannot simply refer to the exclusion curves reported in the experimental papers, in which the contact type interaction is assumed. The different dependencies on $E_{R}$ and $v$ from the contact ones should be taken into account, if the rate is affected by the non-contact contributions. The method to translate the null results at direct detection experiments into limits on the parameter space in our model is explained in appendix B.

In the Dirac DM model, the interactions via the charge radius $b_{\chi}$, the magnetic dipole $\mu_{\chi}$, and electric dipole $d_{\chi}$ are particularly important, since these are suppressed by neither the DM velocity $v \sim 10^{-3}$ nor the nuclear recoil energy $E_{R}=\mathcal{O}(10 \mathrm{keV})$. The anapole $a_{\chi}$ is suppressed by $v$ or $E_{R}$, thus it has a negligible effect in the Dirac case. In the minimal setup, the electric dipole $d_{\chi}$ is also vanishing. The contribution from the photon penguin diagram in figure 6 (left) is proportional to $\left|\lambda_{L}^{i}\right|^{2}$ in the case (i) or $\left|\lambda_{R}^{i}\right|^{2}$ in the case (ii), which is CP-even, whereas $d_{\chi}$ is $\mathrm{CP}$-violating. Thus, the latter cannot be generated in the minimal setups. In the non-minimal models with both double and singlet mediators, there is a non-vanishing $d_{\chi}$ proportional to $\operatorname{Im}\left(\lambda_{L}^{i} \lambda_{R}^{i}\right)$. The asymptotic behaviors of $b_{\chi}$ and $\mu_{\chi}$, as $m_{\widetilde{L}} \rightarrow \infty$, are given by

$$
\begin{aligned}
& b_{\chi} \simeq \frac{e Q_{f}}{96 \pi^{2} m_{\widetilde{L}}^{2}} \sum_{i}\left|\lambda_{L}^{i}\right|^{2}\left(\frac{3}{2}+\log \frac{m_{i}^{2}}{m_{\widetilde{L}}^{2}}\right), \\
& \mu_{\chi} \simeq-\frac{e Q_{f} m_{\chi}}{64 \pi^{2} m_{\widetilde{L}}^{2}} \sum_{i}\left|\lambda_{L}^{i}\right|^{2}
\end{aligned}
$$

in the case (i). The expression of the case (ii) is obtained by $\lambda_{L}^{i} \rightarrow \lambda_{R}^{i *}$ and $m_{\widetilde{L}} \rightarrow m_{\widetilde{E}}$. There appears a logarithmic enhancement in $b_{\chi}$ as in the complex DM, while such an enhancement is absent in $\mu_{\chi}$. As pointed out in ref. [21], the charge radius operator gives dominant contribution to the scattering rate for $m_{\chi} \lesssim 1 \mathrm{TeV}$, while the magnetic dipole operator becomes dominant one for the larger masses. Since the former is a dimension six operator and the latter is a dimension five, their asymptotic behaviors in large $m_{\chi}$ scale as $1 / m_{\chi}^{2}$ and $1 / m_{\chi}$, respectively. As a consequence, the magnetic dipole interaction remains more relevant than the charge radius operator, as $m_{\chi} \rightarrow \infty$.

For the Majorana DM, only the anapole moment $a_{\chi}$ is non-vanishing in eq. (4.11) due to the Majorana condition, $\chi^{c}=\chi$. The differential cross section is obtained by setting $b_{\chi}=\mu_{\chi}=d_{\chi}=0$ and replacing $a_{\chi} \rightarrow 2 a_{\chi}$ in eq. (4.13). The reason for the latter replacement is that there are twice as many possible contributing diagrams as the Dirac DM. The $Z$ and Higgs penguin contributions are also non-vanishing, but these are suppressed by lepton masses and thus negligible. The direct detection bounds on the Majorana DM case can be obtained by the same method as in the Dirac DM, although these are essentially very weak due to the suppressions by the DM velocity $v$ and the nuclear recoil energy $E_{R}$. 


\subsection{Current status}

In this section, we summarize current limits on the minimal models. Free parameters in the models are the DM mass, the mediator mass and the three portal Yukawa couplings $\lambda_{L(R)}^{i}$. We assume that one of the three portal couplings is sizable and the other two are negligibly small such that LFV processes are suppressed. In this section, we show plots in the muon-philic case, $\lambda_{L(R)}^{\mu} \gg \lambda_{L(R)}^{e, \tau}$. The qualitative behavior is similar to that of the electron- and tau-philic cases. For simplicity, the Yukawa couplings to the muon $\lambda_{L, R}^{\mu}$ are simply denoted by $\lambda_{L, R}$ in the following.

Figures 7 and 8 show the limits from the direct and indirect detection as well as new physics contribution to the muon anomalous magnetic moment $\Delta a_{\mu}$ and a scale of Landau pole in the four types of DM models. The coupling $\lambda_{L}$ (or $\lambda_{R}$ ) is fixed to explain the central value of the observed DM abundance: $\Omega_{\mathrm{CDM}} h^{2}=0.1186 \pm 0.0020$ [30]. We define a scale of Landau pole $\Lambda$ where $\lambda_{L(R)}(\Lambda)=\sqrt{4 \pi}$. The beta functions for the Yukawa coupling constants are listed in appendix C. Similar study can be found in ref. [86] for the real DM, in ref. [29] for the complex DM, in refs. [20, 43] for the Majorana DM and in ref. [21] for the Dirac DM.

In these figures, the latest result of the XENON1T experiment [107] excludes the red region. In the gray region on the upper corner, the DM thermal abundance requires a non-perturbatively large coupling $\lambda_{L(R)}>\sqrt{4 \pi}$ at the freeze-out. In the green region, coannihilation is too efficient to explain the thermal abundance observed by the Planck collaboration [30]. The orange contours stand for the Landau pole scales and the purple contours stand for $\Delta a_{\mu}$. The brown regions are excluded by the slepton searches at the LHC, where the limits are projected from figure 2 .

The shaded blue regions in the bottom panels are excluded by the current gamma line observations at the Fermi-LAT [108] and the HESS [109]. The cyan lines show the future sensitivity at the GAMAM-400 [92] and the CTA [93]. To obtain these limits, we refer to the $95 \%$ C.L. upper limit on the combined cross section $\langle\sigma v\rangle_{\ell \bar{\ell} \gamma}+2\langle\sigma v\rangle_{\gamma \gamma}$ shown in figure 5 of [82], where the Einasto density profile is assumed:

$$
\rho_{\mathrm{DM}}(r) \propto \exp \left\{-\frac{2}{\alpha_{E}}\left(\frac{r}{r_{s}}\right)^{\alpha_{E}}\right\}
$$

with $\alpha_{E}=0.17$ and scale radius $r_{s}=20 \mathrm{kpc}$, normalized to $\rho_{\mathrm{DM}}(r=8.5 \mathrm{kpc})=$ $0.4 \mathrm{GeV} / \mathrm{cm}^{3}$. The astrophysical J-factor associated with the optimized region of interest (ROI) for the DM density profile is $8.48 \times 10^{22} \mathrm{GeV}^{2} / \mathrm{cm}^{5}$ in the Fermi-LAT analysis [108]. For the HESS data, the search region consists of a cone with $1^{\circ}$ radius around the galactic center (GC), while excluding the galactic plane by requiring $|b|>0.3^{\circ}[109]$. Note that the referred limit is obtained in the Majorana DM model with $r=1.1$ and may not be able to apply to the real DM model. As shown in appendix A, however, the photon spectrum of $X X \rightarrow \ell \bar{\ell} \gamma$ in the real DM model is the same as that of $\chi \chi \rightarrow \overline{\ell \ell} \gamma$ in the Majorana DM. In addition, the $X X \rightarrow \bar{\ell} \gamma \gamma$ process dominates over the $X X \rightarrow \gamma \gamma$ process for $r \lesssim 3$ as shown in figure 4. Thus, we apply the upper limit on the Majorana DM to the real DM, assuming that the difference between two limits is marginal as far as $r \lesssim 3$. Indeed, for larger $r$, the models are in the non-perturbative regime, and our analysis is not valid. In the following, we discuss more details of the current limits type-by-type. 

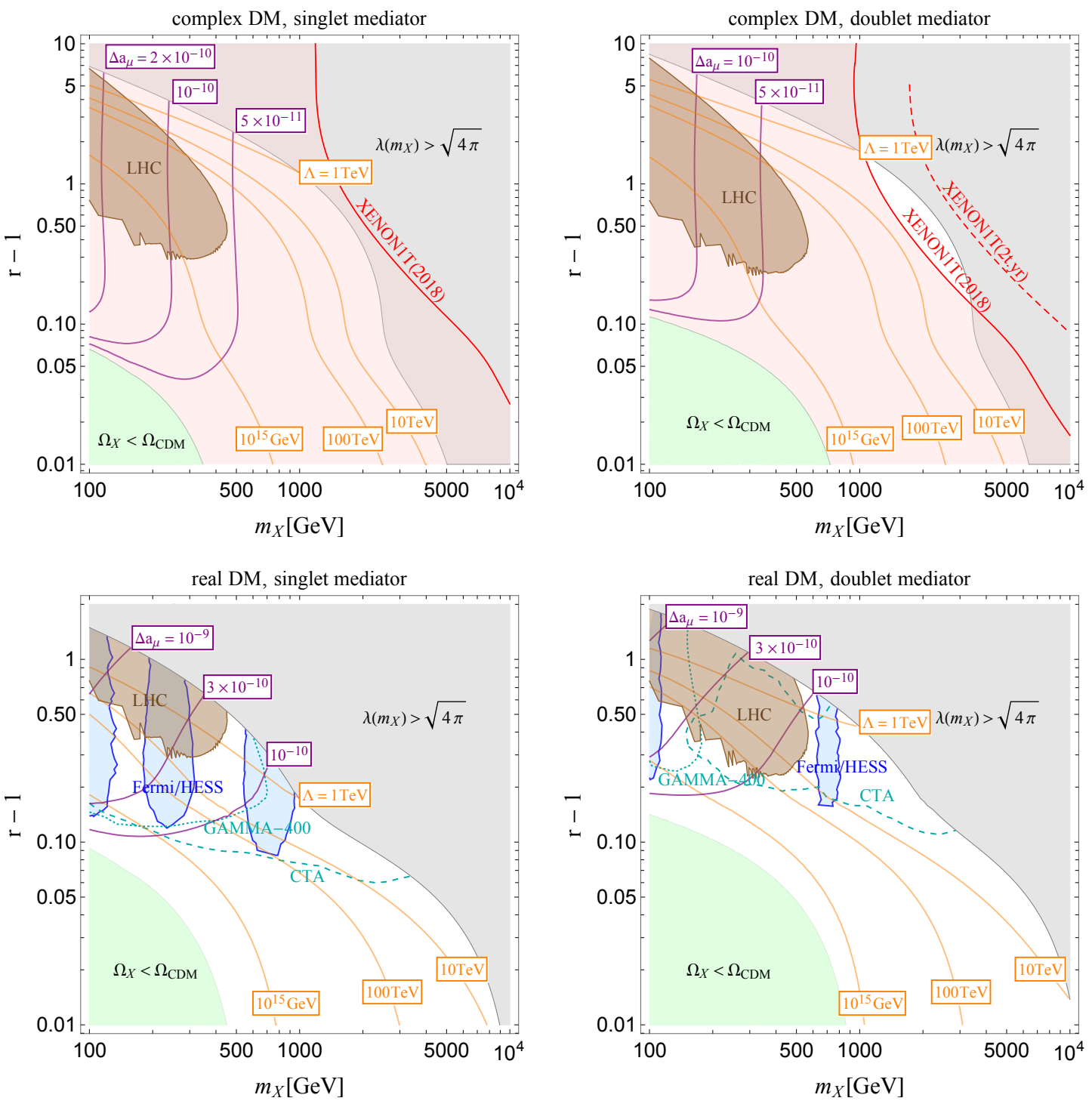

Figure 7. Plots for the complex (top panels) and real (bottom panels) scalar DM. The vectorlike lepton is a weak singlet (left panels) or doublet (right panels). Direct detections of the DM at the XENON1T [107] excludes the red region. In the gray region on the upper corner, the DM thermal abundance requires a non-perturbatively large coupling $\lambda_{L(R)}>\sqrt{4 \pi}$ at the freeze-out. In the green region, coannihilation is too efficient to explain the thermal abundance observed at the Planck [30]. The orange contours stand for the Landau pole scales and the purple contours stand for $\Delta a_{\mu}$. The brown regions are excluded by the slepton searches at the LHC, where the limits are projected from figure 2. The shaded blue regions in the bottom panels are excluded by the current gamma line observations at the Fermi-LAT [108] and the HESS [109]. The cyan lines show the future sensitivity at the GAMAM-400 (dotted) [92] and the CTA (dashed) [93]. 
Complex scalar DM. The two top panels in figure 7 show the results of the complex DM. The two-body annihilation $X X^{\dagger} \rightarrow \ell \bar{\ell}$ is the leading one at the freeze-out. The Yukawa coupling is required to be $\mathcal{O}(1)$ in order to explain the DM abundance, since the $s$ wave contribution is helicity suppressed and the $p$-wave is dominant. The other processes, such as the VIB process $X X^{\dagger} \rightarrow \ell \bar{\ell} V$, are sub-leading and less than $10 \%$ of the total rate, as mentioned above. The large Yukawa coupling lowers the Landau pole scale. For instance, the DM mass should be smaller than $1 \mathrm{TeV}$ in order that the Yukawa couplings are perturbative up to the GUT scale around $10^{16} \mathrm{GeV}$. In compressed regions with $r \sim 1$, the coannihilation further reduces the thermal abundance and the smaller Yukawa coupling $\lambda_{L, R}$ is enough to explain the observed value.

The direct detection at the XENON1T [107] has already excluded wide parameter space. In particular, it fully covers the theoretically allowed parameter space with the singlet vectorlike lepton. It should be noted that the compressed mass region $\left(m_{F} \approx m_{X}\right)$ looks already excluded by the XENON experiment in the figure. As pointed out in ref. [29], allowing $\mathcal{O}(1 \%)$ fine-tuning between $m_{X}$ and $m_{F}$, one should be able to find a narrow allowed region in this mass regime. In this paper, we do not focus on such a fine-tuned case. For readers who are interested in the fine-tuned case, see e.g. ref. [29]. In the $\mathrm{SU}(2)_{L}$ doublet vectorlike lepton case, we can find the allowed region where $1 \mathrm{TeV} \lesssim m_{X} \lesssim 3 \mathrm{TeV}$. It will be covered by the future XENON1T result, whose projected limit is shown by the dashed red line, assuming the sensitivity is 4.5 times better than the current one. The direct detection limit on the tau-philic case will be slightly weaker than that in figure 7 , because the logarithmic factor $\ln m_{\tau} / m_{F}$ is smaller than that of the muon-philic case. The indirect detection is not sensitive to this type of DM as discussed in section 4.3.

The discrepancy of the muon anomalous magnetic moment is hardly explained in this case. As mentioned in section 2.1, the sizable $\Delta a_{\mu}$ can be obtained only if it is enhanced by the vectorlike lepton mass due to the chirality flip. This is a common feature in the minimal lepton portal DM models, irrespectively of DM type. This fact motivates us to consider models with both singlet and doublet mediators.

Real scalar DM. The bottom panels in figure 7 show the results of the real DM model. Both the $s$ - and $p$-wave contributions in the two-body annihilation $X X \rightarrow \ell \bar{\ell}$ are helicity suppressed, so that the $d$-wave is the dominant. The VIB processes $X X \rightarrow \overline{\ell \ell} V$ and the loop processes $V V^{\prime}$ have also too small cross sections to be dominant. As a result, the annihilation rates at the freeze-out temperature are so small that the observed DM abundance can only be explained when $m_{L(E)} \leq 3 m_{X}$ while keeping the perturbative coupling. In the wide parameter space, the DM abundance is correctly produced with the help of coannihilation.

At the DM direct detection, there is no contribution from the photon penguin diagram, unlike the complex DM. The leading DM-nucleon scattering is induced at the 2-loop level via diphoton exchange [101]. As a result, the direct detection gives no constraints. On the other hand, the real DM can be probed by using cosmic ray fluxes at the indirect detection experiments. As discussed in section 4.3, the sharp spectral feature of gamma rays is the promising signal. The blue regions are excluded by the Fermi-LAT [108] and HESS [109]. 

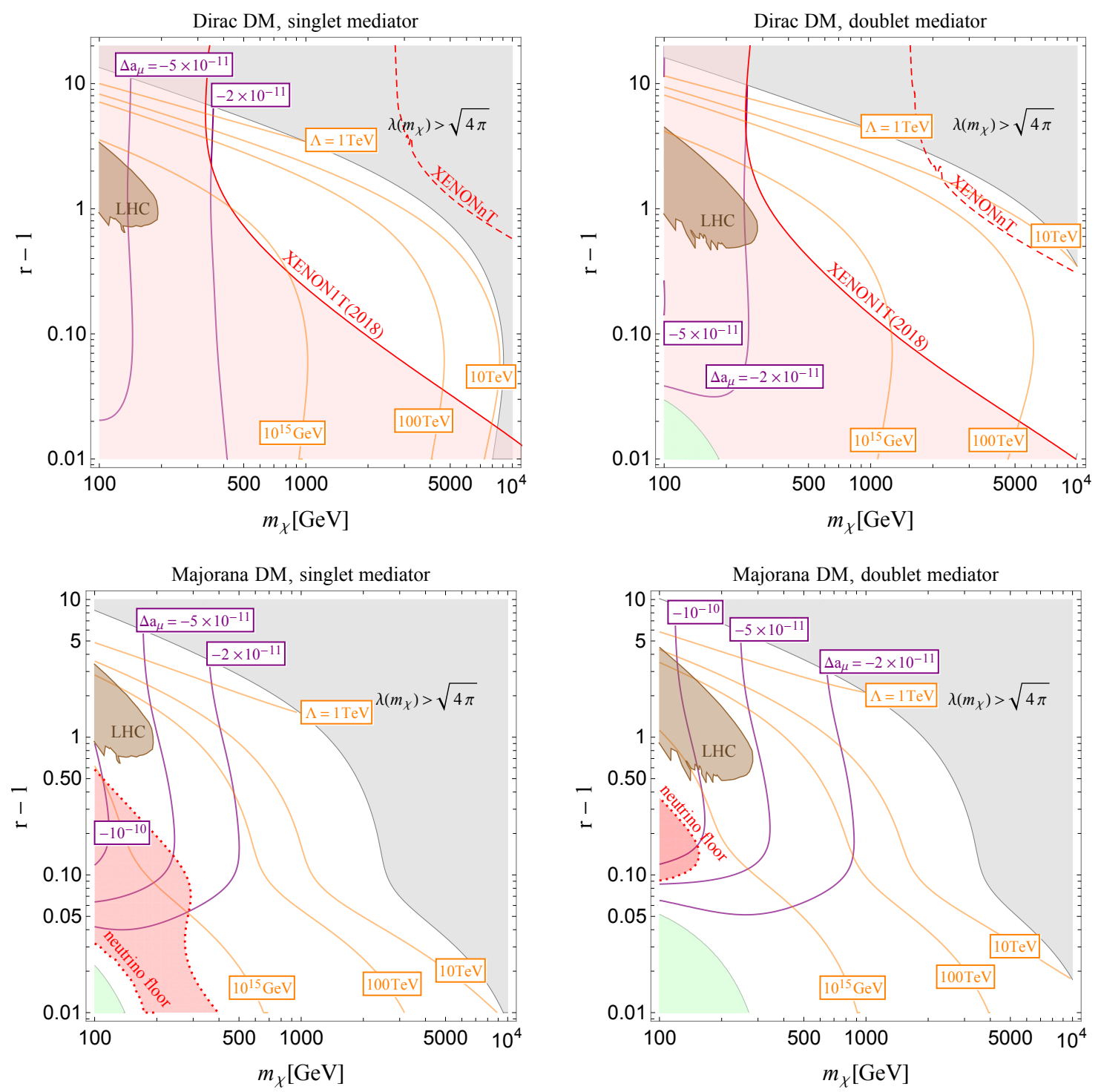

Figure 8. Plots for the fermion DM models with the same constraints as in figure 7 . The top (bottom) panels are for the Dirac (Majorana) fermion DM and the left (right) panels are for the singlet (doublet) slepton.

A combination of future observations at the GAMMA-400 [92] and CTA [110] would test the $\mathrm{SU}(2)_{L}$ singlet vectorlike lepton case when $r \gtrsim 1.1$. The sensitivity to the $\mathrm{SU}(2)_{L}$ doublet vectorlike lepton case is slightly weaker, because $\langle\sigma v\rangle_{\ell \bar{\ell} \gamma}+2\langle\sigma v\rangle_{\gamma \gamma}$ is smaller than the singlet case.

Dirac fermion DM. The results of the Dirac fermion DM case are shown on the top panels in figure 8 . The prime difference from the other types is that the partial $s$-wave in $\chi \bar{\chi} \rightarrow \ell \bar{\ell}$ annihilation is not helicity suppressed. The smaller Yukawa coupling is predicted to account for the DM abundance, and thus the Landau pole scale is higher than the other cases. 
The constraints from the DM direct detection are much weaker than those in the complex DM because of the smaller Yukawa coupling. The current limits on the DM mass from the XENON1T are 300 (220) GeV for the singlet (doublet) slepton case. The XENONnT experiment [111] will probe the region below the red dashed lines and will cover most of the parameter space with the perturbative Yukawa coupling. The expected limit by the XENONnT is obtained by assuming that the future sensitivity is 50 times better than the current limit.

There are no limits from the indirect searches in the muon-philic case as shown in figure 8. The limit from the observations of the dSph galaxies at the Fermi-LAT [94] is about $10 \mathrm{GeV}$ in the muon-philic case, while it reaches about $100 \mathrm{GeV}$ in the tau-philic case. In the electron-philic case, the stringent limit of about $100 \mathrm{GeV}$ will be set by the search for the positron flux originated from the annihilation into $e^{+} e^{-}$.

Majorana fermion DM. The pair annihilation of the Majorana DM is similar to that of the complex DM. The $p$-wave in $\chi \chi \rightarrow \ell \bar{\ell}$ dominates the freeze-out processes. The VIB and loop annihilations are sub-dominant and are no more than $10 \%$ of the total contribution. Hence, sizable Yukawa couplings are required to achieve the correct DM density. This causes lower Landau-pole scales as in the complex DM.

The constraint from the DM direct detection is, on the other hand, so weak that there are no exclusion lines in the figures. At one-loop level, the DM-nuclei scattering arises due to the anapole interaction, $Z$-penguin and Higgs exchanging. The anapole interaction induces the spin-independent scattering. This gives the leading contribution although it is suppressed by the DM velocity. The $Z$-penguin induces contact-type interactions: $\left(\bar{\chi} \gamma_{\mu} \gamma_{5} \chi\right)\left(\bar{N} \gamma^{\mu} N\right)$ and $\left(\bar{\chi} \gamma_{\mu} \gamma_{5} \chi\right)\left(\bar{N} \gamma^{\mu} \gamma_{5} N\right)$. The former contributes to the spinindependent scattering and the latter to the spin-dependent one. These interactions, however, give only sub-leading effects due to the suppression by the lepton mass. The former contribution is further suppressed by the DM velocity. The Higgs exchanging contribution is also suppressed by the leptons mass. Altogether, the current sensitivity of the direct detection cannot reveal the Majorana DM. The red dotted lines correspond to the neutrino floor, which is assumed to be 10 times better than the XENONnT sensitivity. The nuclear recoil rate is above the neutrino floor in the shaded region between the dotted lines.

Moreover, the indirect detection hardly constrains the parameter space since the $\chi \chi \rightarrow$ $\ell \bar{\ell}$ process is suppressed by the small DM velocity $v \sim 10^{-3}$ in our Galaxy. It might be possible that the photon sharp spectral features from the VIB and loop processes are probed at the gamma ray telescopes if a boost factor is $\mathcal{O}(10)$ or larger [82]. We conclude that the Majorana DM is the most invisible from the DM searches among the minimal lepton portal DM models.

\section{Lepton portal DM models for $\Delta a_{\mu}$}

We have seen that it is hard to explain the discrepancy of the muon $g-2$ in the minimal lepton portal DM models that have either $\mathrm{SU}(2)_{L}$ doublet or singlet mediator. In this section, we study the extended models with both doublet and singlet mediators, and 
examine correlations between the DM physics and $\Delta a_{\mu}$. We focus on the real scalar and Majorana fermion DM, since otherwise most of parameter space will be excluded by the DM direct detection.

\subsection{Real scalar DM}

The presence of the vectorlike lepton mixing influences the DM annihilation. In particular, the $s$-wave contribution to $X X \rightarrow e_{i} \bar{e}_{j}$ appears as

$$
(\sigma v)_{e_{i} \bar{e}_{j}}=\frac{\left|\lambda_{L}^{i} \lambda_{R}^{j}\right|^{2}+\left|\lambda_{L}^{j} \lambda_{R}^{i}\right|^{2}}{2 \pi}\left(\frac{c_{R} s_{L} m_{E_{1}}}{m_{X}^{2}+m_{E_{1}}^{2}}-\frac{c_{L} s_{R} m_{E_{2}}}{m_{X}^{2}+m_{E_{2}}^{2}}\right)^{2}+\mathcal{O}\left(v^{2}, \epsilon_{i}\right) .
$$

This is not suppressed by the light SM lepton masses and can dominate the annihilation cross section. Note that the annihilation into a neutrino pair is not changed because of the absence of singlet vectorlike neutrino in our model.

Let us discuss correlation between $\Delta a_{\mu}$ and the DM abundance. First of all, we assume for simplicity that the DM abundance is determined solely by eq. (5.1), i.e. the $s$-wave contribution of $X X \rightarrow \mu \bar{\mu}$, and then estimate the induced $\Delta a_{\mu}$. This assumption implies that the two portal couplings are not hierarchical $\left(\lambda_{L} \simeq \lambda_{R}\right)$, the doublet-singlet mixing is not tiny, and any coannihilation process is not effective. Hence, we may regard $r=m_{E_{1}} / m_{X}$ as not close to unity in this estimate. In this case, based on eq. (5.1), the total annihilation cross section is approximately evaluated as

$$
\sigma_{0} \sim(\sigma v)_{\mu \bar{\mu}} \sim \frac{\left(\lambda_{L}^{\mu} \lambda_{R}^{\mu}\right)^{2}\left(c_{R} s_{L} m_{E_{2}}-c_{L} s_{R} m_{E_{1}}\right)^{2}}{2 \pi m_{E_{1}}^{2} m_{E_{2}}^{2}}
$$

where $\sigma_{0} \simeq 3 \times 10^{-26}\left[\mathrm{~cm}^{3} / \mathrm{s}\right]$ is the canonical value to explain the observed DM density. Inserting this relation into eq. (2.6), we obtain

$$
\Delta a_{\mu} \sim \frac{m_{\mu}}{16 \pi^{2}} \frac{\lambda_{L}^{\mu} \lambda_{R}^{\mu}\left(c_{R} s_{L} m_{E_{2}}-c_{L} s_{R} m_{E_{1}}\right)}{m_{E_{1}} m_{E_{2}}} \sim \frac{m_{\mu}}{16 \pi^{2}} \sqrt{2 \pi \sigma_{0}} .
$$

Thus, $\Delta a_{\mu} \sim 5.0 \times 10^{-8}$ is predicted, which is clearly too large. This conclusion is independent of the DM and mediator masses, the portal Yukawa couplings and mixing angle, as far as the partial $s$-wave of $X X \rightarrow \mu \bar{\mu}$ is responsible for the DM production. This conclusion of too large $\Delta a_{\mu}$ is quite robust. This will not be altered by taking into account sub-dominant effects neglected in eqs. (5.2) and (5.3), such as the finite $r$ correction and the loop functions in $\Delta a_{\mu}$. In order to explain the discrepancy in $\Delta a_{\mu}$, we have to relax some of the above assumptions so that the $s$-wave contribution given by eq. (5.1) does not dominate the DM annihilation. One simple way to do that is to employ sizable coannihilation by a considerable tuning between $m_{X}$ and $m_{E}$. This allows us to suppress $\Delta a_{\mu}$ due to the smaller Yukawa couplings because of the smaller pair-annihilation contributions.

Another way is to make the $d$-wave contribution sizable compared with that of the $s$ wave. This can be achieved by considering hierarchy in $\lambda_{L}$ and $\lambda_{R}$ or smaller mixing angles between the vectorlike leptons. It is obvious from eq. (5.1) that the $s$-wave contribution is proportional to $\left(\lambda_{L} \lambda_{R}\right)^{2}$. If we take $\lambda_{L}=\varepsilon \lambda_{R}(\varepsilon \ll 1)$, the contribution is suppressed by a 

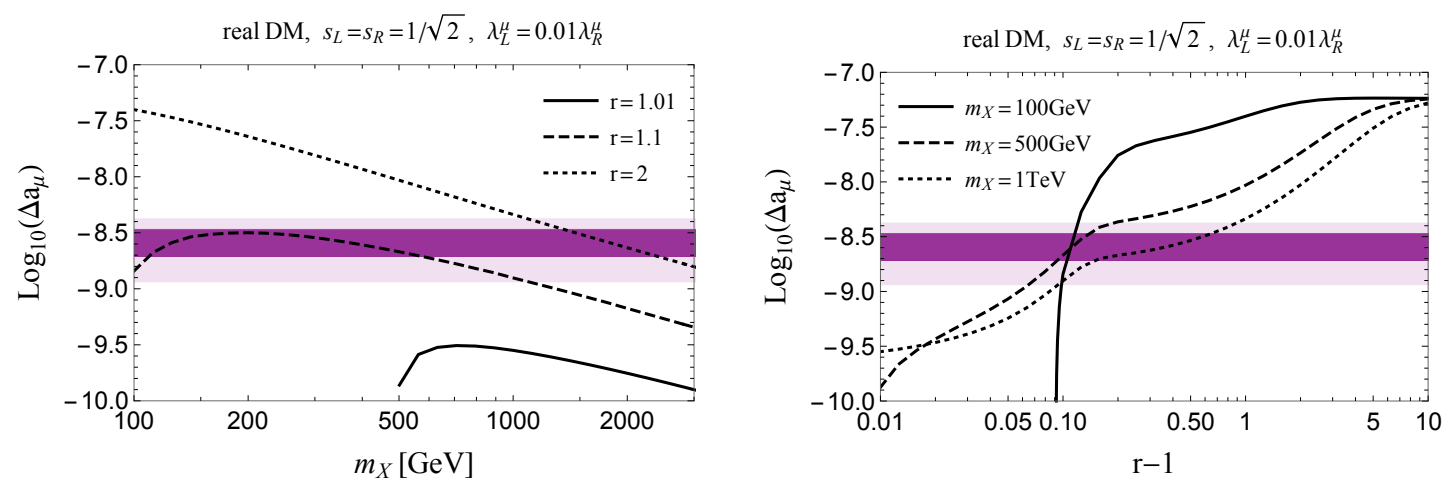

Figure 9. $\Delta a_{\mu}$ in the real scalar DM model as functions of the DM mass $m_{X}$ and degeneracy $r-1$, where $r \equiv m_{E_{1}} / m_{X}$. The solid, dashed and dotted lines are $r=1.01,1.1$ and 2 on the left panel and $m_{X}=100,500 \mathrm{GeV}$ and $1 \mathrm{TeV}$ on the right panel. The Yukawa coupling $\lambda_{L}=0.01 \lambda_{R}$ is fixed via the thermal relic abundance. $\Delta a_{\mu}$ is explained within $1 \sigma(2 \sigma)$ uncertainties on the (light) purple band. We assume the maximal mixing, $s_{L}=s_{R}=1 / \sqrt{2}$ and $\lambda_{L}^{\mu}=0.01 \lambda_{R}^{\mu}$.

factor of $\varepsilon^{2}$. The relative importance of the partial $d$-wave therefore grows as $\varepsilon$ decreases, since the latter has contribution $\propto \lambda_{R}^{4}$ which is not suppressed by $\varepsilon$. The relative growth of the $d$-wave contribution breaks the correlation in eq. (5.3), and the discrepancy of $\Delta a_{\mu}$ can be explained. Similarly, the $s$-wave part is also suppressed by the small mixings, while the $d$-wave is not. In this paper, we will focus on the case with a hierarchical coupling, and will assume the maximal mixing in vectorlike leptons.

To see quantitative details, we show $\Delta a_{\mu}$ in figure 9 as a function of the DM mass $m_{X}$ (left) and the mass ratio $r=m_{E_{1}} / m_{X}$ (right). In the analysis, we assume for simplicity that $m_{L}=m_{E}$ and $\kappa=\tilde{\kappa}$ which lead to the maximal mixings $s_{L}=s_{R}=1 / \sqrt{2}$. The resulting condition $s_{L}=s_{R}$ is also in favor of constraints from the EW precision observables (EWPOs). Furthermore, the mass difference between the vectorlike leptons are set to $m_{E_{2}}-m_{E_{1}}=2 \kappa v_{H}=100 \mathrm{GeV}$. The relative size of the Yukawa couplings is fixed to $\lambda_{L}=0.01 \lambda_{R}$, and the absolute size of the couplings is determined via the observed DM abundance. $\Delta a_{\mu}$ is explained within $1 \sigma(2 \sigma)$ uncertainties on the (light) purple band. We see that $\Delta a_{\mu}$ is successfully explained together with the DM density.

The behavior of $\Delta a_{\mu}$ is understood as follows. With the relation $\lambda_{L}=0.01 \lambda_{R}$, the DM pair annihilation is essentially dominated by the $d$-wave, which scales as $(\sigma v)_{\mu \bar{\mu}} \propto$ $\lambda_{R}^{4} /\left(m_{X}^{2} r^{8}\right)$. Then, as far as coannihilation is irrelevant to the DM production, we find the scaling of $\Delta a_{\mu}$,

$$
\Delta a_{\mu} \sim \frac{m_{\mu} \varepsilon \lambda_{R}^{2} c_{R} s_{L}\left(m_{E_{2}}-m_{E_{1}}\right)}{16 \pi^{2} m_{X}^{2} r^{2}} \propto \frac{\varepsilon r^{2}}{m_{X}}
$$

where we assumed that $\lambda_{R}$ is determined via the DM abundance. It follows from the equation that $\Delta a_{\mu}$ decreases (increases) as the DM mass $m_{X}$ increases (decreases) in this regime. In figure 9 (left), we can observe such a behavior in fact. Once the coannihilation operates and becomes superior to the pair annihilation, smaller Yukawa couplings are predicted to explain the DM abundance and thus $\Delta a_{\mu}$ becomes small. Since the coannihilation is effective as $r \rightarrow 1, \Delta a_{\mu}$ is smaller as $r$ is closer to unity. We see this effect in the solid 
$(r=1.01)$ and dashed $(r=1.1)$ lines in figure 9 (left). As the DM mass decreases, $\Delta a_{\mu}$ increases until $m_{X}=700(200) \mathrm{GeV}$ for $r=1.01$ (1.1), and then $\Delta a_{\mu}$ starts decreasing due to the coannihilation dominance. This behavior cannot be found when $r=2$, because the coannihilation is not effective when $r \gtrsim 1.2$.

We see the effect of the coannihilation more explicitly in figure 9 (right). With the DM mass fixed, a small $\Delta a_{\mu}$ is induced when $r \approx 1$, while $\Delta a_{\mu}$ increases monotonically as $r$ goes away from unity. Remarkably, as $r \rightarrow \infty, \Delta a_{\mu}$ looks approaching the asymptotic value eq. (5.3), independently of the DM mass. To understand that, we shall take a closer look at the DM pair annihilation. Keeping only the relevant contributions, we find the cross section, in the limit of $r \gg 1$ and $\varepsilon \ll 1$,

$$
(\sigma v)_{\mu \bar{\mu}} \simeq \frac{\lambda_{R}^{4}}{8 \pi m_{X}^{2} r^{4}}\left(\frac{\varepsilon^{2}\left(m_{E_{2}}-m_{E_{1}}\right)^{2}}{m_{X}^{2}}+\frac{2 v^{4}}{15 r^{4}}\right)
$$

Despite of the small $\varepsilon$, the $s$-wave contribution can overcome the $d$-wave one, if the following condition is satisfied:

$$
\frac{\varepsilon^{2}\left(m_{E_{2}}-m_{E_{1}}\right)^{2}}{m_{X}^{2}} \gtrsim \frac{2 v^{4}}{15 r^{4}}
$$

In figure 9 (right), we fix $m_{E_{2}}-m_{E_{1}}=100 \mathrm{GeV}$, and $\lambda_{L}=0.01 \lambda_{R}$. Thus, with $m_{X}=$ $100 \mathrm{GeV}$ and the DM velocity at the freeze-out, $v^{4} \sim 0.1$, the above inequality can be satisfied for $r \gtrsim 3$. Once the $s$-wave contribution dominates the DM production, we can repeat the previous discussion to derive eq. (5.3), and then arrive at the asymptotic value $\Delta a_{\mu} \sim 5.0 \times 10^{-8}$.

Figure 10 shows the parameter space, where $\Delta a_{\mu}$ is explained consistently with the DM physics, on the $\left(m_{X}, r-1\right)$ plane (top) and the $\left(m_{X}, \lambda_{L}^{\mu} / \lambda_{R}^{\mu}\right)$ plane (bottom). The size of the Yukawa couplings is fixed to explain the DM abundance. The purple lines show the induced values of $\Delta a_{\mu}$. We also highlight the regions with the (light) purple band, where $\Delta a_{\mu}$ is explained within $1 \sigma(2 \sigma)$. The color-coding of the other regions is the same as in figures 7 and 8 . Since $\Delta a_{\mu}$ can easily be reduced by relaxing our assumption for the maximal mixing, we conclude that the discrepancy $\Delta a_{\mu}$ can be resolved in the real DM model. Note, however, $\mathcal{O}(10 \%)$ mild tunings may be required to realize the DM abundance.

We comment on the constraint from the EWPOs. In the non-minimal model, the EW doublet and singlet vectorlike fermions mix each other. The mixing of these new particles with different EW quantum number could have impacts on the precision observables. We particularly study the observables concerned with the $T$ parameter and the partial $Z$ boson decay width to $\mu \bar{\mu}$. We estimate the bound on the EWPOs based on the study in refs. [30,45]. We conclude that the most stringent constraint comes from the partial decay width of $Z$ boson to $\mu \bar{\mu}$. The deviation of the decay width of $Z \rightarrow \mu \bar{\mu}$ from the $\mathrm{SM}$ prediction is much less than $\mathcal{O}(0.1) \%$ which is below the current limit [30], in the case with $s_{L}=s_{R}=1 / \sqrt{2}$. If $s_{L}$ is not the same as $s_{R}$, the deviation is enhanced and may be tested by the future experiment [45]. The same conclusion of the EWPOs is obtained in the Majorana DM case. 

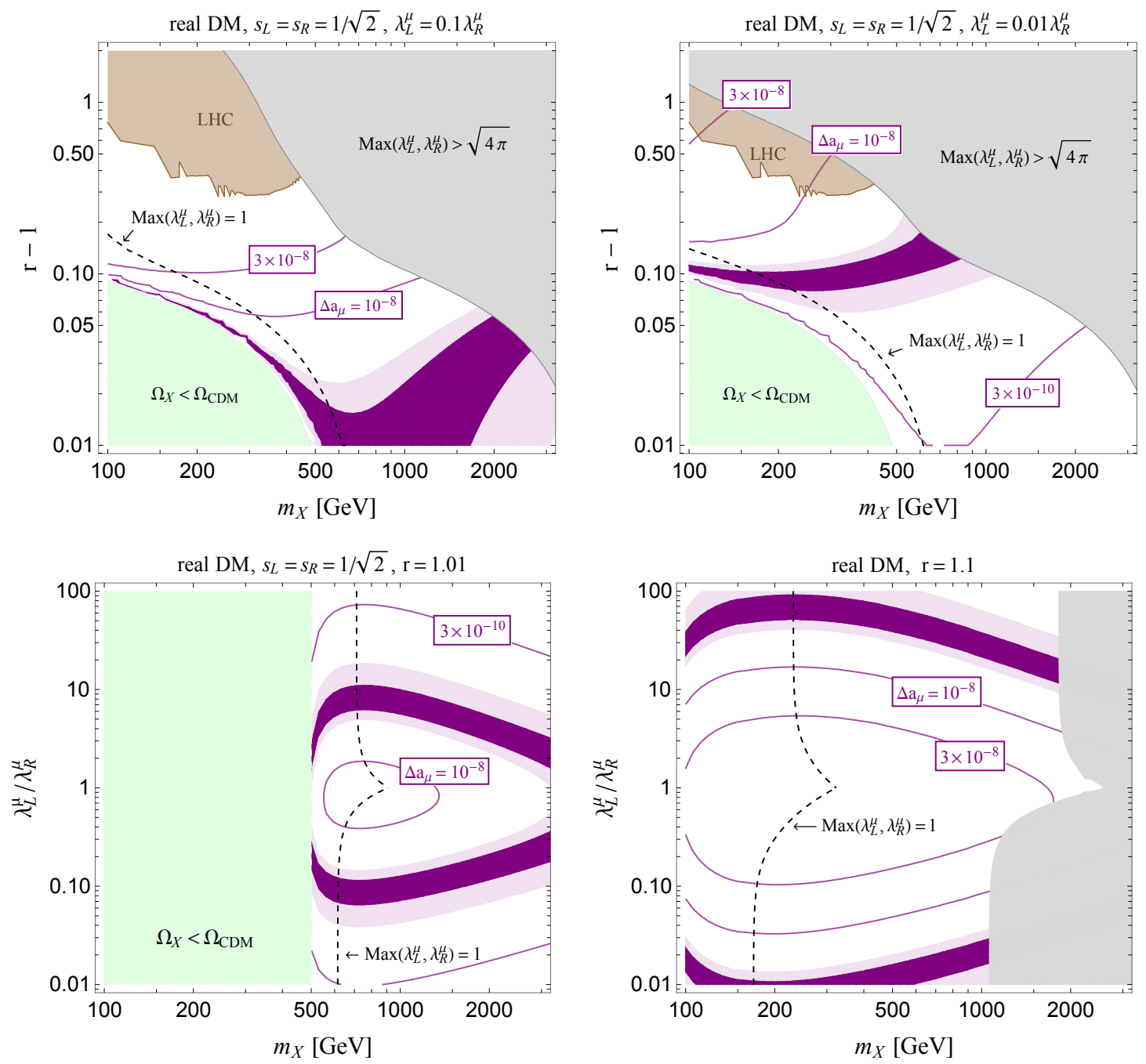

Figure 10. Parameter space consistent with the DM observations in the real DM model with the maximally mixed vectorlike leptons $s_{L}=s_{R}=1 / \sqrt{2}$. We show the induced values of $\Delta a_{\mu}$ with the purple lines. $\Delta a_{\mu}$ is explained in the (light) purple region within $1 \sigma(2 \sigma)$.

\subsection{Majorana fermion DM}

As in the real DM model, the annihilation $\chi \chi \rightarrow e_{i} \bar{e}_{j}$ has the $s$-wave contribution,

$$
(\sigma v)_{e_{i} \bar{e}_{j}}=\frac{\left|\lambda_{L}^{i} \lambda_{R}^{j}\right|^{2}+\left|\lambda_{L}^{j} \lambda_{R}^{i}\right|^{2}}{16 \pi}\left(\frac{c_{\theta} s_{\theta} m_{\chi}}{m_{\chi}^{2}+m_{\widetilde{E}_{1}}^{2}}-\frac{c_{\theta} s_{\theta} m_{\chi}}{m_{\chi}^{2}+m_{\widetilde{E}_{2}}^{2}}\right)^{2}+\mathcal{O}\left(v^{2}, \epsilon_{i}\right),
$$

that is not suppressed by the lepton masses.

Let us study the correlation between the DM physics and $\Delta a_{\mu}$ in the Majorana DM model. In this case, the DM pair annihilation $\chi \chi \rightarrow \mu \bar{\mu}$ will dominantly contribute to the DM production as far as the DM is enough lighter than the sleptons and the coannihilation is negligible. We neglect these effects for simplicity. In this case, the annihilation cross section is given by

$$
(\sigma v)_{\mu \bar{\mu}} \sim \frac{\left(c_{\theta} s_{\theta} \lambda_{L}^{\mu} \lambda_{R}^{\mu}\right)^{2}}{16 \pi} \frac{\left(m_{\widetilde{E}_{2}}^{2}-m_{\widetilde{E}_{1}}^{2}\right)^{2}}{m_{\chi}^{6} r^{8}}+\frac{\left(\lambda_{L}^{\mu}\right)^{4}+\left(\lambda_{R}^{\mu}\right)^{4}}{48 \pi m_{\chi}^{2} r^{4}} v^{2},
$$



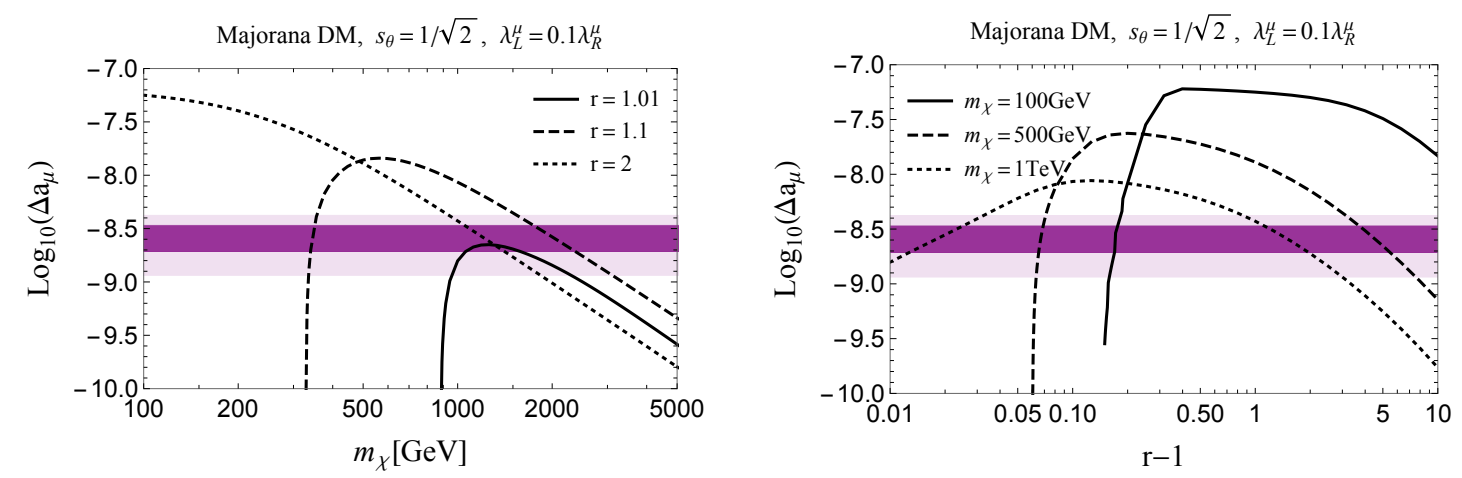

Figure 11. The same plots for the Majorana DM as figure 9.

with $r=m_{\widetilde{E}_{1}} / m_{\chi}$. Here, the second term is the $p$-wave contribution from the chiralityconserving interaction. If the $s$-wave part is dominant, $\Delta a_{\mu}$ is estimated as

$$
\Delta a_{\mu} \sim \frac{m_{\mu}}{16 \pi^{2}} \frac{c_{\theta} s_{\theta} \lambda_{L} \lambda_{R}\left(m_{\widetilde{E}_{2}}^{2}-m_{\widetilde{E}_{1}}^{2}\right)}{m_{\chi}^{3} r^{4}} \sim \frac{m_{\mu}}{16 \pi^{2}} \sqrt{16 \pi \sigma_{0}},
$$

where we used the fact that the cross section is approximately equal to $\sigma_{0}$. Thus, if the above assumption is valid, $\Delta a_{\mu} \sim 10^{-7}$ is predicted as in the real DM model.

The Majorana case, however, is not so simple as the real scalar case. Even if $\lambda_{L}=\lambda_{R}$ and the maximal mixing $s_{\theta}=1 / \sqrt{2}$, the $p$-wave can have comparable contribution with the $s$-wave, in spite of a mild velocity suppression $v^{2} \sim 0.24$. The cross section in this case is expressed by

$$
(\sigma v)_{\mu \bar{\mu}} \sim \frac{\left(\lambda_{R}^{\mu}\right)^{4}}{64 \pi m_{\chi}^{2} r^{4}}\left(\frac{\left(m_{\widetilde{E}_{2}}^{2}-m_{\widetilde{E}_{1}}^{2}\right)^{2}}{m_{\chi}^{4} r^{4}}+\frac{8 v^{2}}{3}\right) .
$$

As $r$ increases, the $s$-wave contribution is decaying more rapidly than the $p$-wave. This indicates that the latter can still be leading for large $r$. For example, when we consider the $100 \mathrm{GeV} \mathrm{DM}$ and the slepton mass difference $m_{\widetilde{E}_{2}}^{2}-m_{\widetilde{E}_{1}}^{2}=(100 \mathrm{GeV})^{2}$, the two contributions have the similar size at $r \simeq 1.1$. With such a parameter set, the $p$-wave will therefore be leading contribution where $r \gtrsim 1.1$. Note that $r=1.1$ is not large, so that the coannihilation is operative to some extent in this regime. It may be illuminating to derive an asymptotic behavior in the Majorana DM case as well as the real scalar one. In general, both the $s$ - and $p$-wave contributions are comparably important, but in the limit of $r \rightarrow \infty$ or $m_{\chi} \rightarrow \infty$, we find that the $p$-wave is dominant. In this limit, $\Delta a_{\mu}$ is evaluated by

$$
\Delta a_{\mu} \sim \frac{m_{\mu} c_{\theta} s_{\theta}\left(m_{\widetilde{E}_{2}}^{2}-m_{\widetilde{E}_{1}}^{2}\right)}{16 \pi^{2} m_{\chi}^{2} r^{2}} \sqrt{\frac{\left(\lambda_{L} / \lambda_{R}\right)^{2}}{1+\left(\lambda_{L} / \lambda_{R}\right)^{4}}} \sqrt{\frac{48 \pi \sigma_{0}}{v^{2}}} .
$$

The expression in the Majorana DM case is more complicated than that in the real DM case. $\Delta a_{\mu}$ depends on $m_{\chi}, r, \lambda_{L} / \lambda_{R}$, the slepton mixing and the slepton mass difference.

Figure 11 shows $\Delta a_{\mu}$ as a function of the DM mass $m_{\chi}$ and the ratio $r \equiv m_{\widetilde{E}_{1}} / m_{\chi}$. As in the real DM case, we consider $m_{\widetilde{L}}^{2}=m_{\widetilde{E}}^{2}$, so that the slepton mixing is maximized, $s_{\theta}=$ $1 / \sqrt{2}$. The trilinear coupling $A$ in eq. $(2.10)$ is set to $A v_{H}=(500 \mathrm{GeV})^{2}=\left(m_{\widetilde{E}_{2}}^{2}-m_{\widetilde{E}_{1}}^{2}\right) / 2$. 

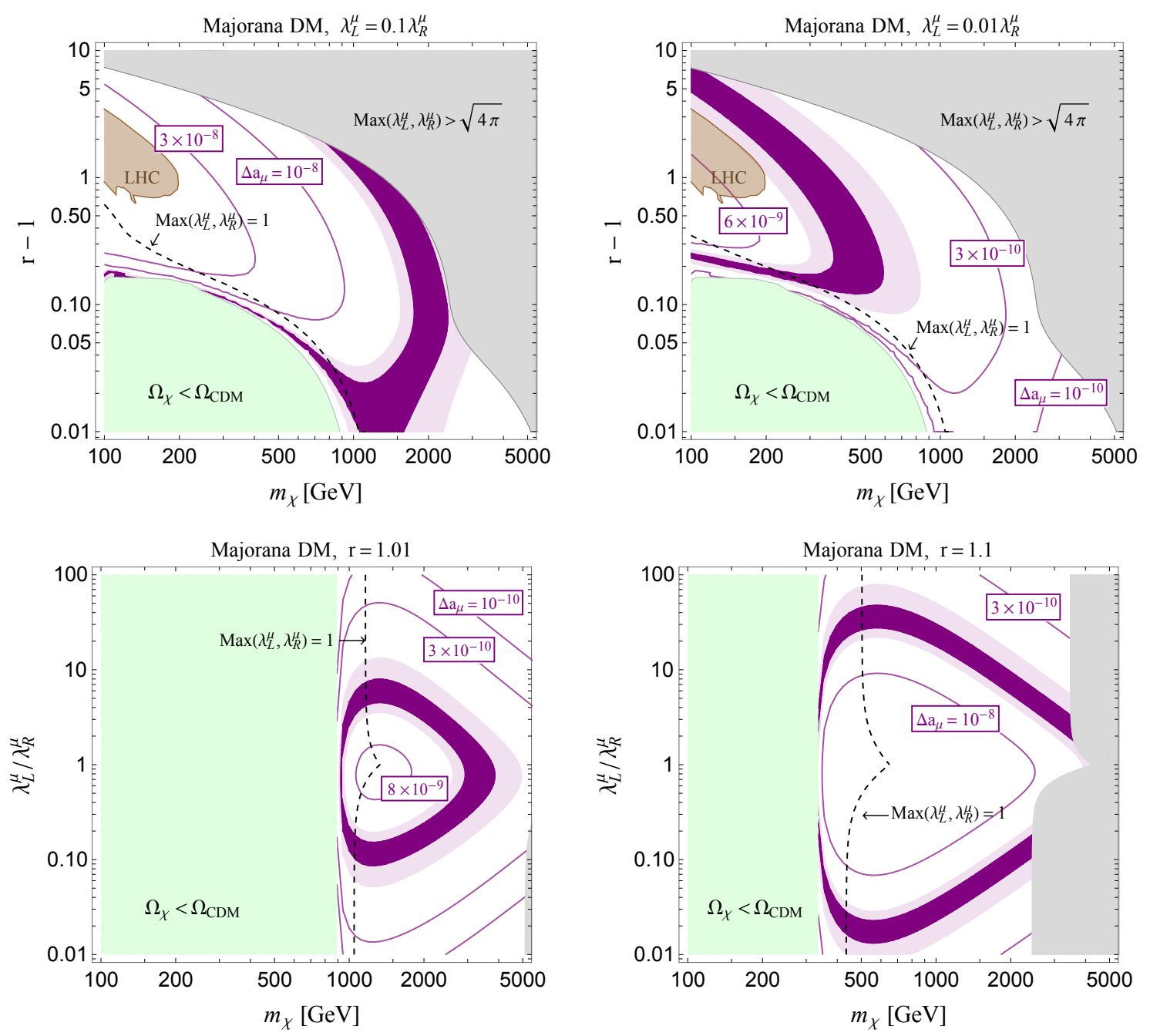

Figure 12. The same plots for the Majorana DM as figure 10.

The ratio of the two portal couplings is $\lambda_{L}=0.1 \lambda_{R}$, and the absolute size is determined to explain the DM density. We see that $\Delta a_{\mu}>\mathcal{O}\left(10^{-9}\right)$ can be realized where the DM is lighter than a few $\mathrm{TeV}$.

The dependence on the DM mass and the ratio $r$ is slightly different from that in the real DM case. Concerning the DM mass dependence, as $m_{\chi}$ increases, $\Delta a_{\mu}$ decreases more rapidly than in the real DM. This is understood by comparing eqs. (5.11) and (5.4). $\Delta a_{\mu} \propto 1 / m_{\chi}^{2}$ in the Majorana case, while $\Delta a_{\mu} \propto 1 / m_{\chi}$ in the real DM case. $\Delta a_{\mu}$ increases with decreasing $m_{\chi}$, but when the coannihilation becomes active, $\Delta a_{\mu}$ starts decreasing. This behavior is similar to the real DM case. Further, we can see in figure 11 (right) that the dependence on $r$ is also different. Since $\Delta a_{\mu}$ is scaling as $1 / r^{2}$ for large $r, \Delta a_{\mu}$ decreases with increasing $r$ in the Majorana case. That is in contrast to the real DM case where $\Delta a_{\mu}$ is almost independent of $r$ in the large $r$ limit. With decreasing $r, \Delta a_{\mu}$ is increasing, but when $r$ gets close to unity, the coannihilation becomes effective, which makes the portal Yukawa couplings small. Then, eq. (5.11) is no longer valid. Altogether, $\Delta a_{\mu}$ is maximized at $r-1=0.1-1$ as shown in figure 11 . 
Figure 12 is the same figure as figure 10 but in the Majorana DM case. We choose the same parameter set as in figure 11. The degeneracy of the mediator and DM masses are still required in the Majorana case, but the condition is slightly relaxed. For instance, $\left(m_{\widetilde{E}_{1}}-m_{\chi}\right) / m_{\chi}=\mathcal{O}(1)$ is allowed when $\lambda_{L} / \lambda_{R}=0.1$ as shown in the top-left of figure 12 . As a consequence, we can accommodate both DM and $(g-2)_{\mu}$ explanations without terrible fine-tuning in the masses and the hierarchical couplings, but this time we have to cost a very large Yukawa coupling close to $\sqrt{4 \pi}$, which in turn causes a low Landau pole scale.

\section{Summary}

In this paper, we have examined the lepton portal DM models, in which scalar or fermion DM couples to SM leptons via the Yukawa interactions involving the DM and leptons. New EW charged fields, namely vectorlike leptons or sleptons, are introduced to realize the Yukawa coupling at the renormalizable level. Depending on the spin of the DM, the new EW charged fields are different. We have classified our DM models as shown in table 1 and discussed the phenomenology in each model. The DM relic abundance can be achieved thermally in all the DM models. One important issue is how we can evade strong bounds from DM direct detection experiments, without spoiling the thermal production of DM. We have found that the complex DM model has been almost excluded by the latest XENON1T result. In the Dirac DM model, the $s$-wave contribution is dominant in the annihilation, so the bound from the DM direct detection is relatively weak, and several hundreds GeV DM is still allowed. The future sensitivity of the XENONnT/LZ experiments will probe the remaining parameter space in the Dirac model. The real DM and the Majorana DM, on the other hand, could evade the direct detection bound, although small mass difference between the DM and the extra EW charged particle is required to evade too low Landau pole scale. One interesting point is that the real DM model can be tested by the indirect detection.

We have also investigated a possibility that the lepton portal DM models can explain the discrepancy in the muon anomalous magnetic moment, together with the DM thermal production. We have found that sufficiently large $\Delta a_{\mu}$ cannot be induced in the minimal models, where either singlet or doublet vectorlike lepton (or slepton) exists. This consequence is common to the four DM types. When the extended model with both singlet and doublet is considered, $\Delta a_{\mu}$ can be easily accommodated. In section 5 , we have demonstrated this possibility in both real and Majorana DM models, and clarified explicit correlations between the annihilation cross section of DM and $\Delta a_{\mu}$, as shown in eqs. (5.3) and (5.11). If there is a DM particle behind the discrepancy, there are both $\mathrm{SU}(2)_{L}$ doublet and singlet fields in addition to the DM, and their couplings with the DM are large. As far as detectability is concerned, it may be difficult to prove the explanations in both cases, depending on the parameter space. If $\mathrm{DM}$ is lighter than a few $\mathrm{TeV}$ and the mass difference is larger than $10 \%$, the indirect search for DM probably concludes the real DM scenario. The LHC experiments possibly prove both real and Majorana DM scenarios, if the DM mass is several hundreds of $\mathrm{GeV}$. In other parameter region, we may be able to test our scenarios in flavor physics, turning on the other couplings between the DM and other leptons. 
In our study, we have focused on the heavy DM region, $m_{\mathrm{DM}} \geq 100 \mathrm{GeV}$. There would be allowed regions, even if the DM is lighter than EW gauge bosons, since the direct detection experiments become insensitive to the light DM region. In such a light DM case, however, it may be difficult to achieve the relic abundance of the DM thermally, due to the lower limits on masses of the EW charged new particles from the collider experiments. The resulting large mass splitting between the DM and the new charged particles gives rise to the inactive coannihilation mechanism. Without the coannihilation, the DM production relies on the DM pair annihilation, whose cross section behaves as $\langle\sigma v\rangle \propto \lambda^{4} m_{\mathrm{DM}}^{2} / m_{E}^{4}$. It is easy to see that the DM cannot be so light when $m_{E} \gtrsim 100 \mathrm{GeV}$ while keeping perturbativity. This suggests that the thermal production of the DM will impose a lower limit on the DM mass. In addition, as the DM gets lighter, the cosmological and astrophysical searches, such as the CMB observation and the indirect detection, will grow in importance. The bounds depend on the detail of DM models and need a further dedicated analysis, that is beyond the scope of this paper.

\section{Acknowledgments}

This work is supported in part by the Grant-in-Aid for Scientific Research from the Ministry of Education, Science, Sports and Culture (MEXT), Japan No. 18K13534 (J.K.), No. 19H04614, No. 19H05101 and No. 19 K03867 (Y.O.). The work of J.K. is supported in part by the Department of Energy (DOE) under Award No. DE-SC0011726. The work of S.O. is supported in part by NSERC, Canada, and JSPS Overseas Challenge Program for Young Researchers.

\section{A Analytic expressions}

In this appendix, we summarize analytic expressions and intermediate results, which have been omitted in the main text. We focus on the minimal models, and consider only the case that DM couples to the left-handed leptons $\ell^{i}$, introducing the vectorlike doublet $L(\widetilde{L})$, whose neutral and charge component are assumed to be degenerate $m_{E}=m_{N}\left(m_{\widetilde{E}}=m_{\widetilde{N}}\right)$. The results can easily be translated into the case of right-handed leptons $e_{R}^{i}$, by replacing $\lambda_{L}^{i} \rightarrow \lambda_{R}^{* i}$ and taking the weak charges of leptons into account. For abbreviation, we use $\mu \equiv m_{L}^{2} / m_{X}^{2}$ and $\epsilon_{i} \equiv m_{i}^{2} / m_{X}^{2}$. In a part of calculation of 1-loop annihilations into $V V^{\prime}$, we exploit Package-X_2.0 [112].

\section{A.1 Complex scalar DM}

For scalar DM, the relevant interaction is given by

$$
\mathcal{L}_{S}=-\lambda_{L}^{* i} X^{\dagger} \bar{L}_{R} \ell_{L}^{i}+\text { h.c. }
$$

where $\ell^{i}=e^{i}, \nu_{L}^{i}$ denote charged leptons or neutrinos.

\section{A.1.1 Annihilation}

1. $\boldsymbol{X} \boldsymbol{X}^{\dagger} \rightarrow \ell^{i} \bar{\ell}^{j}$. For the velocity expansion,

$$
(\sigma v)_{X X^{\dagger} \rightarrow \ell^{i} \bar{\ell}^{j}}=a_{i j}+b_{i j} v^{2},
$$


we find

$$
\begin{aligned}
a_{i j} & =\frac{\left(\epsilon_{i}+\epsilon_{j}\right)}{32 \pi m_{X}^{2}(1+\mu)^{2}}\left|\lambda_{L}^{i} \lambda_{L}^{j}\right|^{2}, \\
b_{i j} & =\frac{\left|\lambda_{L}^{i} \lambda_{L}^{j}\right|^{2}}{48 \pi m_{X}^{2}(1+\mu)^{2}},
\end{aligned}
$$

where we only keep the leading order terms in $\epsilon_{i}$ and $\epsilon_{j}$.

2. $\boldsymbol{X} \boldsymbol{X}^{\dagger} \rightarrow \ell^{i} \bar{\ell}^{j} \boldsymbol{V}$. The differential cross section is expressed by

$$
v d \sigma_{\ell^{i} \bar{\ell}^{j} V}=\frac{\left|\mathcal{M}_{V}\right|^{2}}{128 \pi^{3}} d x d y,
$$

where $x \equiv 2 E_{V} / \sqrt{s}$ and $y \equiv 2 E_{f} / \sqrt{s}$. The cross section is obtained by performing $x$ and $y$ integrals over $\sqrt{\xi_{V}} \leq x \leq 1+\xi_{V} / 4$ and $y_{-} \leq y \leq y_{+}$, where $\xi_{V} \equiv m_{V}^{2} / m_{X}^{2}(V=Z, W, h)$ and

$$
y_{ \pm}=\frac{1}{2}\left(2-x \pm \sqrt{x^{2}-\xi_{V}}\right) .
$$

The calculation has been done in the $s$-wave limit and we neglect lepton masses.

Squared amplitudes:

$$
\begin{aligned}
\left|\mathcal{M}_{\gamma}^{S_{c}}\right|^{2} & =\frac{32 \pi \alpha Q_{f}^{2}\left|\lambda_{L}^{i} \lambda_{L}^{j}\right|^{2}}{m_{X}^{2}} f_{\gamma}^{S_{c}}(x, y) \\
\left|\mathcal{M}_{Z}^{S_{c}}\right|^{2} & =\frac{32 \pi \alpha\left|\lambda_{L}^{i} \lambda_{L}^{j}\right|^{2}}{m_{X}^{2}} \frac{\left(\left(T_{3}\right)_{\ell_{L}}-Q_{\ell} s_{W}^{2}\right)^{2}}{c_{W}^{2} s_{W}^{2}} f_{Z}^{S_{c}}(x, y) \\
\left|\mathcal{M}_{W}^{S_{c}}\right|^{2} & =\frac{32 \pi \alpha\left|\lambda_{L}^{i} \lambda_{L}^{j}\right|^{2}}{m_{X}^{2} s_{W}^{2}} f_{W}^{S_{c}}(x, y) \\
\left|\mathcal{M}_{h}^{S_{c}}\right|^{2} & =\frac{\left|\lambda_{L}^{i} \lambda_{L}^{j}\right|^{2}}{m_{X}^{2}}\left[\left(\frac{m_{i}}{v}\right)^{2} \frac{1-x+\xi_{h} / 4}{(3+\mu-2 x-2 y)^{2}}+\left(\frac{m_{j}}{v}\right)^{2} \frac{1-x+\xi_{h} / 4}{(1-\mu-2 y)^{2}}\right],
\end{aligned}
$$

where we mean $\ell^{i} \bar{\ell}^{j} W=e^{i} \bar{\nu}^{j} W^{+}+\bar{e}^{i} \nu^{j} W^{-}$and

$$
f_{V}^{S_{c}}(x, y)=\frac{(1-x)\left(2-2 x+x^{2}-4 y+2 x y+2 y^{2}\right)+\frac{\xi_{V}}{4}\left(x^{2}-2 x+2\right)}{(1-\mu-2 y)^{2}(3+\mu-2 x-2 y)^{2}} .
$$

Differential cross sections:

$$
\begin{aligned}
\frac{d(\sigma v)_{\ell^{i} \bar{\ell}^{j} \gamma}^{S_{c}}}{d x}= & \frac{\alpha Q_{\ell}^{2}\left|\lambda_{L}^{i} \lambda_{L}^{j}\right|^{2}}{16 \pi^{2} m_{X}^{2}}(1-x)\left[\frac{2 x}{(1+\mu)(1+\mu-2 x)}-\frac{x}{(1+\mu-x)^{2}}\right. \\
& \left.-\frac{(1+\mu)(1+\mu-2 x)}{2(1+\mu-x)^{3}} \log \left(\frac{1+\mu}{1+\mu-2 x}\right)\right] \\
\frac{d(\sigma v)_{\ell^{i} \bar{\ell}^{j} Z}^{S_{C}}}{d x}= & \frac{\alpha\left|\lambda_{L}^{i} \lambda_{L}^{j}\right|^{2}}{16 \pi^{2} m_{X}^{2}} \frac{\left(\left(T_{3}\right)_{\ell_{L}}-Q_{\ell} s_{W}^{2}\right)^{2}}{c_{W}^{2} s_{W}^{2}} g_{Z}^{S_{c}}(x), \\
\frac{d(\sigma v)_{\ell^{i} \bar{\ell}^{j} W}^{S_{c}}}{d x}= & \frac{\alpha\left|\lambda_{L}^{i} \lambda_{L}^{j}\right|^{2}}{16 \pi^{2} m_{X}^{2} s_{W}^{2}} g_{W}^{S_{c}}(x), \\
\frac{d(\sigma v)_{\ell^{i} \bar{\ell}^{j} h}}{d x}= & \frac{\left|\lambda_{L}^{i} \lambda_{L}^{j}\right|^{2}}{128 \pi^{3} m_{X}^{2}} \frac{m_{i}^{2}+m_{j}^{2}}{v^{2}} \frac{\left(1-x+\xi_{h} / 4\right) \sqrt{x^{2}-\xi_{h}}}{(1+\mu)(1+\mu-2 x)+\xi_{h}}
\end{aligned}
$$


where

$$
\begin{aligned}
g_{V}^{S_{c}}(x)= & \frac{2(1-x) \sqrt{x^{2}-\xi_{V}}}{(1+\mu)(1+\mu-2 x)+\xi_{V}}-\frac{(1-x) \sqrt{x^{2}-\xi_{V}}}{(1+\mu-x)^{2}} \\
& -\frac{(1-x)(1+\mu)(1+\mu-2 x)}{(1+\mu-x)^{3}} \tanh ^{-1}\left(\frac{\sqrt{x^{2}-\xi_{V}}}{1+\mu-x}\right)+\frac{\xi_{V}}{2(1+\mu-x)^{2}} \\
& \times\left\{\frac{(x-2)^{2} \sqrt{x^{2}-\xi_{V}}}{(1+\mu)(1+\mu-2 x)+\xi_{V}}+\frac{x^{2}-2 x+2}{1+\mu-x} \tanh ^{-1}\left(\frac{\sqrt{x^{2}-\xi_{V}}}{1+\mu-x}\right)\right\} .
\end{aligned}
$$

The results are consistent with $[83,84,86]$.

Cross sections:

$$
\begin{aligned}
(\sigma v)_{\ell^{i} \ell^{j} \gamma}^{S_{c}}= & \frac{\alpha Q_{\ell}^{2}\left|\lambda_{L}^{i} \lambda_{L}^{j}\right|^{2}}{32 \pi^{2} m_{X}^{2}}\left[(1+\mu)\left\{\frac{\pi^{2}}{6}-\log ^{2}\left(\frac{1+\mu}{2 \mu}\right)-2 \operatorname{Li}_{2}\left(\frac{1+\mu}{2 \mu}\right)\right\}\right. \\
& \left.+\frac{4 \mu+3}{\mu+1}+\frac{(4 \mu+1)(\mu-1)}{2 \mu} \log \left(\frac{\mu-1}{\mu+1}\right)\right], \\
(\sigma v)_{\ell^{i} \bar{\ell}^{j} h}^{S_{c}}= & \frac{\left|\lambda_{L}^{i} \lambda_{L}^{j}\right|^{2}}{128 \pi^{3} m_{X}^{2}} \frac{m_{i}^{2}+m_{j}^{2}}{v^{2}} \frac{1}{64(1+\mu)^{3}} \\
& \times\left[(1+\mu)\left(4-\xi_{h}\right)\left\{4 \mu^{2}+\mu\left(4-\xi_{h}\right)+3 r_{h}\right\}\right. \\
& -4(\mu-1)\left(2+2 \mu-\xi_{h}\right)\left\{(1+\mu)^{2}-\xi_{h}\right\} \log \left(\frac{2+2 \mu-\xi_{h}}{2(\mu-1)}\right) \\
& \left.+4 \xi_{h}\left\{\mu\left(4+\xi_{h}\right)+4-\xi_{h}\right\} \log \left(\frac{\xi_{h}}{4}\right)\right],
\end{aligned}
$$

where $\operatorname{Li}_{2}(z)=-\int_{0}^{1} d t \log (1-z t) / t$ is the dilogarithm function. The cross sections for the $Z$ and $W$ emissions are obtained by numerical integrations.

3. $X X^{\dagger} \rightarrow V V^{\prime}$.

$$
\begin{aligned}
(\sigma v)_{\gamma \gamma}^{S_{c}} & =\frac{\alpha^{2} Q_{e}^{4}}{128 \pi^{3} m_{X}^{2}}\left(\sum_{i}\left|\lambda_{L}^{i}\right|^{2}\right)^{2}\left[2+\operatorname{Li}_{2}\left(\frac{1}{\mu}\right)-\operatorname{Li}_{2}\left(-\frac{1}{\mu}\right)-2 \mu \arcsin ^{2}\left(\frac{1}{\sqrt{\mu}}\right)\right]^{2}, \\
(\sigma v)_{\gamma Z}^{S_{c}} & =\frac{\alpha^{2} Q_{e}^{2}\left(\left(T_{3}\right)_{e_{L}}-Q_{e} s_{W}^{2}\right)^{2}}{64 \pi^{3} m_{X}^{2} c_{W}^{2} s_{W}^{2}}\left(\sum_{i}\left|\lambda_{L}^{i}\right|^{2}\right)^{2}\left(1-\frac{\xi_{Z}}{4}\right)\left|A_{\gamma Z}^{S}\right|^{2}, \\
(\sigma v)_{Z Z}^{S_{c}} & =\frac{\alpha^{2}}{512 \pi^{3} m_{X}^{2} c_{W}^{4} s_{W}^{4}}\left(\sum_{i} \sum_{\ell=e, \nu_{L}}\left|\lambda_{L}^{i}\right|^{2}\left(\left(T_{3}\right)_{\ell_{L}}-Q_{\ell} s_{W}^{2}\right)^{2}\right)^{2} \sqrt{1-\xi_{Z}}\left|A_{Z Z}^{S}\right|^{2} \\
(\sigma v)_{W W}^{S_{c}} & =\frac{\alpha^{2}}{1024 \pi^{3} m_{X}^{2} s_{W}^{4}}\left(\sum_{i}\left|\lambda_{L}^{i}\right|^{2}\right)^{2} \sqrt{1-\xi_{W}}\left|A_{W W}^{S}\right|^{2}
\end{aligned}
$$

where $A_{\gamma Z}^{S}$ is

$$
\begin{aligned}
A_{\gamma Z}^{S}= & 2-\frac{\xi_{Z}}{4-\xi_{Z}}\left[\frac{4 \mu}{\sqrt{\mu-1}} \operatorname{arccot}(\sqrt{\mu-1})+2 \log \left(\frac{\mu-1}{\mu}\right)\right. \\
& \left.-2 \sqrt{\frac{4 \mu}{\xi_{Z}}-1} \operatorname{arccot}\left(\sqrt{\frac{4 \mu}{\xi_{Z}}-1}\right)-\log \left(\frac{\xi_{Z}}{\mu}\right)+i \pi\right]
\end{aligned}
$$




$$
\begin{aligned}
& +m_{X}^{2}\left\{\frac{\mu\left(4-4 \mu-\xi_{Z}\right)}{1-\mu} C_{0}\left(m_{Z}^{2}, 4 m_{X}^{2}, 0 ; m_{L}^{2}, m_{L}^{2}, m_{L}^{2}\right)\right. \\
& +\frac{\mu\left(4+\xi_{Z}\right)\left(-2+2 \mu+\xi_{Z}\right)}{2(1-\mu)\left(4 \mu+\xi_{Z}\right)} C_{0}\left(\frac{m_{Z}^{2}}{2}-m_{X}^{2}, m_{X}^{2}, 0 ; m_{L}^{2}, 0, m_{L}^{2}\right) \\
& +\left[\frac{\xi_{Z}}{4}-\frac{4(1+\mu)}{4-\xi_{Z}}+\frac{4 \mu(1+\mu)}{4 \mu+\xi_{Z}}\right] C_{0}\left(\frac{m_{Z}^{2}}{2}-m_{X}^{2}, m_{X}^{2}, m_{Z}^{2} ; 0, m_{L}^{2}, 0\right) \\
& \left.+\left[\mu+\frac{\xi_{Z}}{2(1-\mu)}-\frac{4(1+\mu)}{4-\xi_{Z}}\right] C_{0}\left(\frac{m_{Z}^{2}}{2}-m_{X}^{2}, m_{X}^{2}, m_{Z}^{2} ; m_{L}^{2}, 0, m_{L}^{2}\right)\right\}
\end{aligned}
$$

where $C_{0}$ is the Passarino-Veltman function defined by

$$
C_{0}\left(p_{1}^{2},\left(p_{1}-p_{2}\right)^{2}, p_{2}^{2} ; m_{1}^{2}, m_{2}^{2}, m_{3}^{2}\right)=\int \frac{d^{4} l}{i \pi^{2}} \frac{1}{l^{2}-m_{1}^{2}} \frac{1}{\left(l+p_{1}\right)^{2}-m_{2}^{2}} \frac{1}{\left(l+p_{2}\right)^{2}-m_{3}^{2}}
$$

The above results are consistent with ref. [86].

The loop function $A_{Z Z}^{S}$ is more complicated. To the best of our knowledge, the explicit expression is given for the first time, which is described by

$$
\left|A_{Z Z}^{S}\right|^{2}=|A|^{2}+2|B|^{2}
$$

where

$$
\begin{aligned}
A= & 4-\frac{\xi_{Z}}{1-\xi_{Z}}\left[\frac{4 \mu}{\sqrt{\mu-1}} \operatorname{arccot}(\sqrt{\mu-1})+2 \log \left(\frac{\mu-1}{\mu}\right)\right. \\
& -2 \sqrt{\frac{4 \mu}{\xi_{Z}}-1} \operatorname{arccot}\left(\sqrt{\left.\frac{4 \mu}{\xi_{Z}}-1\right)}-\log \left(\frac{\xi_{Z}}{\mu}\right)+i \pi\right], \\
& +\frac{\xi_{Z}}{1-\xi_{Z}} m_{X}^{2}\left\{2\left[\frac{4 \mu}{\xi_{Z}}-\frac{\mu\left(4 \mu-6+\xi_{Z}\right)}{(\mu-1)}\right] C_{0}\left(4 m_{X}^{2}, m_{Z}^{2}, m_{Z}^{2} ; m_{L}^{2}, m_{L}^{2}, m_{L}^{2}\right)\right. \\
& -\left[\frac{4}{\xi_{Z}}+\frac{(\mu-1)^{3}+(\mu+1)\left(2-\xi_{Z}\right)}{\mu(\mu-1)}\right] C_{0}\left(m_{X}^{2}, m_{Z}^{2}-m_{X}^{2}, m_{Z}^{2} ; m_{L}^{2}, 0, m_{L}^{2}\right) \\
& \left.-\frac{1+\mu^{2}-\xi_{Z}}{\mu} C_{0}\left(m_{X}^{2}, m_{Z}^{2}-m_{X}^{2}, m_{Z}^{2} ; 0, m_{L}^{2}, 0\right)\right\},
\end{aligned}
$$

and

$$
\begin{aligned}
B= & \frac{\xi_{Z}}{1-\xi_{Z}}\left[\frac{4 \mu}{\sqrt{\mu-1}} \operatorname{arccot}(\sqrt{\mu-1})+2 \log \left(\frac{\mu-1}{\mu}\right)\right. \\
& \left.-2 \sqrt{\frac{4 \mu}{\xi_{Z}}-1} \operatorname{arccot}\left(\sqrt{\frac{4 \mu}{\xi_{Z}}-1}\right)-\log \left(\frac{\xi_{Z}}{\mu}\right)+i \pi\right] \\
& +\frac{\xi_{Z}}{1-\xi_{Z}} m_{X}^{2}\left\{\frac{2 \mu\left(-2+\xi_{Z}\right)}{\mu-1} C_{0}\left(4 m_{X}^{2}, m_{Z}^{2}, m_{Z}^{2} ; m_{L}^{2}, m_{L}^{2}, m_{L}^{2}\right)\right. \\
& +\frac{\mu^{2}-1+\xi_{Z}}{\mu} C_{0}\left(m_{X}^{2}, m_{Z}^{2}-m_{X}^{2}, m_{Z}^{2} ; 0, m_{L}^{2}, 0\right) \\
& \left.+\left[\frac{(\mu-1)^{2}}{\mu}+\frac{4 \mu}{\mu-1}+\frac{\xi_{Z}(1-3 \mu)}{\mu(\mu-1)}\right] C_{0}\left(m_{X}^{2}, m_{Z}^{2}-m_{X}^{2}, m_{Z}^{2} ; m_{L}^{2}, 0, m_{L}^{2}\right)\right\}
\end{aligned}
$$


In the limit of $m_{Z} \rightarrow 0$, the latter function is not contributing $B \approx \mathcal{O}\left(m_{Z}^{2}\right)$, while the former function is approximate to

$$
A \approx 2\left[2+\operatorname{Li}_{2}\left(\frac{1}{\mu}\right)-\operatorname{Li}_{2}\left(-\frac{1}{\mu}\right)-2 \mu \arcsin ^{2}\left(\frac{1}{\sqrt{\mu}}\right)\right]+\mathcal{O}\left(m_{Z}^{2}\right)
$$

which is equivalent to the loop function appearing in the photon contribution eq. (A.15), as it should be. For $W W$, the amplitude is obtained by the replacement $A_{W W}^{S}=\left.A_{Z Z}^{S}\right|_{m_{Z} \rightarrow m_{W}}$.

\section{A.1.2 Direct detection}

Charge radius operator: the photon penguin diagram induces the so-called DM charge radius operator,

$$
\mathcal{L}_{\text {eff }}^{S} \supset b_{X}\left(i X^{\dagger} \overleftrightarrow{\partial_{\mu}} X\right) \partial_{\nu} F^{\mu \nu}
$$

which in turn provides the DM coupling to the quark vector current, $C_{V, q}=-e Q_{q} b_{X}$, through the equation of motion for photon. The penguin contribution involving $L$ and $\ell^{i}$ is given by

$$
\left(b_{X}\right)_{\ell^{i}}=\frac{e Q_{\ell}\left|\lambda_{L}^{i}\right|^{2}}{16 \pi^{2} m_{X}^{2}} \hat{b}_{X}\left(\mu, \epsilon_{i}\right)
$$

Loop functions are

$$
\begin{aligned}
\hat{b}_{X}(\mu, \epsilon)=-\frac{1}{3}\left[\frac{\mu-\epsilon}{\Delta^{3 / 2}}\{(\right. & \mu+\epsilon+1) \Delta-4 \mu \epsilon\} \tanh ^{-1}\left(\frac{\Delta^{1 / 2}}{\mu+\epsilon-1}\right) \\
+ & \left.\frac{2+\mu+\epsilon}{2} \log \left(\frac{\epsilon}{\mu}\right)+\frac{(\mu-\epsilon)(\mu+\epsilon-1)}{\Delta}\right],
\end{aligned}
$$

with $\Delta \equiv \mu^{2}+(\epsilon-1)^{2}-2 \mu(\epsilon+1)$.

$Z$-penguin: the $Z$ penguin diagram also induces the DM coupling to the quark vector current,

$$
\mathcal{L}_{\text {eff }}^{S} \supset C_{V, q}\left(i X^{\dagger} \overleftrightarrow{\partial_{\mu}} X\right)\left(\bar{q} \gamma^{\mu} q\right)
$$

The contribution is expressed by $C_{V, q}^{Z}=\sum_{\ell^{i}}\left(C_{V, q}^{Z}\right)_{\ell_{L}^{i}}$ with

$$
\begin{aligned}
& \left(C_{V, q}^{Z}\right)_{e_{L}^{i}}=\frac{2 \sqrt{2} G_{F} g_{V, q}}{16 \pi^{2} m_{X}^{2}} g_{A, e^{i}}\left|\lambda_{L}^{i}\right|^{2} \hat{a}_{Z}^{S}\left(\mu, \epsilon_{i}\right), \\
& \left(C_{V, q}^{Z}\right)_{\nu_{L}^{i}}=0,
\end{aligned}
$$

with $g_{V, q}=\left(T_{3}\right)_{q}-Q_{q} s_{W}^{2}$ and $g_{A, \ell}=\left(T_{3}\right)_{\ell}$, and the loop function is

$$
\hat{a}_{Z}^{S}(\mu, \epsilon)=\epsilon\left[1+\frac{1+\mu-\epsilon}{2} \log \left(\frac{\epsilon}{\mu}\right)+\frac{\Delta+2 \mu}{\Delta^{1 / 2}} \tanh ^{-1}\left(\frac{\Delta^{1 / 2}}{\mu+\epsilon-1}\right)\right] .
$$

It should be noted that, if DM couples to the singlet leptons $e_{R}^{i}$, the sign of the $Z$ penguin contribution is flipped:

$$
\left(C_{V, q}^{Z}\right)_{e_{R}^{i}}=-\frac{2 \sqrt{2} G_{F} g_{V, q}}{16 \pi^{2} m_{X}^{2}} g_{A, e^{i}}\left|\lambda_{R}^{i}\right|^{2} \hat{a}_{Z}^{S}\left(\mu, \epsilon_{i}\right),
$$

with $g_{A, e^{i}}=-1 / 2$. 


\section{A.2 Real scalar DM}

The notation of Yukawa interaction is the same as the complex case, but DM is selfconjugate in this case $\left(X^{\dagger}=X\right)$.

\section{A.2.1 Annihilation}

1. $\boldsymbol{X} \boldsymbol{X} \rightarrow \ell^{i} \bar{\ell}^{j}$. Similarly, for the following velocity expansion,

$$
(\sigma v)_{X X \rightarrow \ell^{i} \bar{\ell}^{j}}=a_{i j}+b_{i j} v^{2}+c_{i j} v^{4}
$$

the coefficients are

$$
\begin{aligned}
a_{i j} & =\frac{\left(\epsilon_{i}+\epsilon_{j}\right)}{8 \pi m_{X}^{2}(1+\mu)^{2}}\left|\lambda_{L}^{i} \lambda_{L}^{j}\right|^{2}, \\
b_{i j} & =\frac{-(1+2 \mu)\left(\epsilon_{i}+\epsilon_{j}\right)}{12 \pi m_{X}^{2}(1+\mu)^{4}}\left|\lambda_{L}^{i} \lambda_{L}^{j}\right|^{2}, \\
c_{i j} & =\frac{\left|\lambda_{L}^{i} \lambda_{L}^{j}\right|^{2}}{60 \pi m_{X}^{2}(1+\mu)^{4}} .
\end{aligned}
$$

2. $\boldsymbol{X} \boldsymbol{X} \rightarrow \ell^{i} \bar{\ell}^{j} \boldsymbol{V}$ and $\boldsymbol{V} \boldsymbol{V}^{\prime}$. Cross sections for these processes are just four times larger than the corresponding cross sections in the complex DM. The spectrum of vector boson in $X X \rightarrow \ell^{i} \bar{\ell}^{j} V$ and the dependence on $\mu$ and $\xi_{V}$ in both processes are completely same.

\section{A.3 Dirac DM}

The relevant interaction is given by

$$
\mathcal{L}_{F}=-\lambda_{L}^{* i} \widetilde{L}^{\dagger} \bar{\chi}_{R} \ell_{L}^{i}+\text { h.c.. }
$$

All results given here are consistent with ref. [21].

\section{A.3.1 Annihilation}

1. $\chi \bar{\chi} \rightarrow \ell^{i} \bar{\ell}^{j}$

$$
(\sigma v)_{\chi \bar{\chi} \rightarrow \ell^{i} \bar{\ell}^{j}}=\frac{\left|\lambda_{L}^{i} \lambda_{L}^{j}\right|^{2}}{32 \pi m_{\chi}^{2}(1+\mu)^{2}}
$$

\section{A.3.2 Direct detection}

The effective DM interactions to photon are described by

$$
\mathcal{L}_{\text {eff }}^{F}=b_{\chi} \bar{\chi} \gamma^{\mu} \chi \partial^{\nu} F_{\mu \nu}+\frac{\mu_{\chi}}{2} \bar{\chi} \sigma^{\mu \nu} \chi F_{\mu \nu}+a_{\chi} \bar{\chi} \gamma^{\mu} \gamma_{5} \chi \partial^{\nu} F_{\mu \nu}+i \frac{d_{\chi}}{2} \bar{\chi} \sigma^{\mu \nu} \gamma_{5} \chi F_{\mu \nu} .
$$

The contributions involving $\widetilde{L}$ and $\ell^{i}$ are written as follows:

$$
\begin{aligned}
\left(b_{\chi}\right)_{\ell^{i}} & =\frac{e Q_{\ell}\left|\lambda_{L}^{i}\right|^{2}}{16 \pi^{2} m_{\chi}^{2}} \hat{b}_{\chi}\left(\mu, \epsilon_{i}\right), \\
\left(\mu_{\chi}\right)_{\ell^{i}} & =\frac{e Q_{\ell}\left|\lambda_{L}^{i}\right|^{2}}{16 \pi^{2} m_{\chi}} \hat{\mu}_{\chi}\left(\mu, \epsilon_{i}\right), \\
\left(a_{\chi}\right)_{\ell^{i}} & =\frac{e Q_{\ell}\left|\lambda_{L}^{i}\right|^{2}}{16 \pi^{2} m_{\chi}^{2}} \hat{a}_{\chi}\left(\mu, \epsilon_{i}\right), \\
\left(d_{\chi}\right)_{\ell^{i}} & =0 .
\end{aligned}
$$




\section{Charge radius operator:}

$$
\begin{aligned}
\hat{b}_{\chi}(\mu, \epsilon)= & -\frac{1}{24}\left[(8 \mu-8 \epsilon+1) \log \left(\frac{\epsilon}{\mu}\right)+4\left(4+\frac{\mu+3 \epsilon-1}{\Delta}\right)\right. \\
& \left.+\frac{2}{\Delta^{3 / 2}}\left\{8 \Delta^{2}+(9 \mu+7 \epsilon-5) \Delta-4 \epsilon(3 \mu+\epsilon-1)\right\} \tanh ^{-1}\left(\frac{\Delta^{1 / 2}}{\mu+\epsilon-1}\right)\right],
\end{aligned}
$$

Magnetic dipole operator:

$$
\hat{\mu}_{\chi}(\mu, \epsilon)=-\frac{1}{2}\left[\frac{1}{2}(\epsilon-\mu) \log \left(\frac{\epsilon}{\mu}\right)-1-\frac{\Delta+\mu+\epsilon-1}{\Delta^{1 / 2}} \tanh ^{-1}\left(\frac{\Delta^{1 / 2}}{\mu+\epsilon-1}\right)\right],
$$

\section{Anapole operator:}

$$
\hat{a}_{\chi}(\mu, \epsilon)=\frac{1}{12}\left[\frac{3}{2} \log \left(\frac{\epsilon}{\mu}\right)+\frac{3 \mu-3 \epsilon+1}{\Delta^{1 / 2}} \tanh ^{-1}\left(\frac{\Delta^{1 / 2}}{\mu+\epsilon-1}\right)\right]
$$

$Z$-penguin: the couplings to vector and axial vector currents,

$$
\mathcal{L}_{\text {eff }}^{F} \supset C_{V, q}^{Z} \bar{\chi} \gamma_{\mu} \chi \bar{q} \gamma^{\mu} q+C_{A, q}^{Z} \bar{\chi} \gamma_{\mu} \gamma_{5} \chi \bar{q} \gamma^{\mu} \gamma_{5} q,
$$

are induced. The $Z$ penguin contribution involving $\widetilde{L}$ and $\ell^{i}$ is

$$
\begin{aligned}
\left(C_{V, q}^{Z}\right)_{e_{L}^{i}} & =\frac{\sqrt{2} G_{F} g_{V, q}}{16 \pi^{2}} g_{A, e^{i}}\left|\lambda_{L}^{i}\right|^{2} \hat{a}_{Z}^{F}\left(\mu, \epsilon_{i}\right), \\
\left(C_{A, q}^{Z}\right)_{e_{L}^{i}} & =-\frac{\sqrt{2} G_{F} g_{A, q}}{16 \pi^{2}} g_{A, e^{i}}\left|\lambda_{L}^{i}\right|^{2} \hat{a}_{Z}^{F}\left(\mu, \epsilon_{i}\right),
\end{aligned}
$$

and $\left(C_{V, q}^{Z}\right)_{\nu_{L}^{i}}=\left(C_{A, q}^{Z}\right)_{\nu_{L}^{i}}=0$, where the loop function is

$$
\hat{a}_{Z}^{F}(\mu, \epsilon)=\epsilon\left[\frac{1+\mu-\epsilon}{\Delta^{1 / 2}} \tanh ^{-1}\left(\frac{\Delta^{1 / 2}}{\mu+\epsilon-1}\right)+\frac{1}{2} \log \left(\frac{\epsilon}{\mu}\right)\right] .
$$

Like the real DM case, if DM couples to the singlet leptons $e_{R}^{i}$, the sign of the contribution to $C_{V, q}$ should be flipped, while the contribution to $C_{A, q}$ is unchanged:

$$
\begin{aligned}
\left(C_{V, q}^{Z}\right)_{e_{R}^{i}} & =-\frac{\sqrt{2} G_{F} g_{V, q}}{16 \pi^{2}} g_{A, e^{i}}\left|\lambda_{R}^{i}\right|^{2} \hat{a}_{Z}^{F}\left(\mu, \epsilon_{i}\right), \\
\left(C_{A, q}^{Z}\right)_{e_{R}^{i}} & =-\frac{\sqrt{2} G_{F} g_{A, q}}{16 \pi^{2}} g_{A, e^{i}}\left|\lambda_{R}^{i}\right|^{2} \hat{a}_{Z}^{F}\left(\mu, \epsilon_{i}\right) .
\end{aligned}
$$

\section{Higgs exchanging:}

$$
\begin{aligned}
\mathcal{L}_{\text {eff }}^{F} & \supset C_{S, q} m_{q} \bar{\chi} \chi \bar{q} q, \\
\left(C_{S, q}^{\mathrm{Higgs}}\right)_{f^{i}} & =\frac{-\sqrt{2} G_{F} m_{\chi}}{16 \pi^{2} m_{h}^{2}}\left|\lambda_{L}^{i}\right|^{2} \hat{c}_{H}\left(\mu, \epsilon_{i}\right),
\end{aligned}
$$

with

$$
\hat{c}_{H}(r, \epsilon)=\epsilon\left[\frac{\Delta+\mu+\epsilon-1}{\Delta^{1 / 2}} \tanh ^{-1}\left(\frac{\Delta^{1 / 2}}{\mu+\epsilon-1}\right)+1+\frac{\mu-\epsilon}{2} \log \left(\frac{\epsilon}{\mu}\right)\right] .
$$




\section{A.4 Majorana DM}

The notation of the Yukawa interaction in the Majorana case is the same as that in the Dirac case.

\section{A.4.1 Annihilation}

1. $\chi \chi \rightarrow \ell^{i} \bar{\ell}^{j}$.

$$
(\sigma v)_{\chi \chi \rightarrow \ell^{i} \bar{\ell}^{j}}=a_{i j}+b_{i j} v^{2}
$$

with

$$
\begin{aligned}
a_{i j} & =\frac{\left(\epsilon_{i}+\epsilon_{j}\right)}{64 \pi m_{\chi}^{2}(1+\mu)^{2}}\left|\lambda_{L}^{i} \lambda_{L}^{j}\right|^{2}, \\
b_{i j} & =\frac{\left(1+\mu^{2}\right)}{48 \pi m_{\chi}^{2}(1+\mu)^{4}}\left|\lambda_{L}^{i} \lambda_{L}^{j}\right|^{2} .
\end{aligned}
$$

2. $\chi \chi \rightarrow \ell^{i} \bar{\ell}^{j} V$.

Squared amplitudes:

$$
\begin{aligned}
& \left|\mathcal{M}_{\gamma}^{F_{r}}\right|^{2}=\frac{1}{2}\left|\mathcal{M}_{\gamma}^{S_{c}}\right|^{2}, \\
& \left|\mathcal{M}_{Z}^{F_{r}}\right|^{2}=\frac{16 \pi \alpha\left|\lambda_{L}^{i} \lambda_{L}^{j}\right|^{2}}{m_{\chi}^{2}} \frac{\left(\left(T_{3}\right)_{\ell_{L}}-Q_{\ell} s_{W}^{2}\right)^{2}}{c_{W}^{2} s_{W}^{2}} f_{Z}^{F_{r}}(x, y), \\
& \left|\mathcal{M}_{W}^{F_{r}}\right|^{2}=\frac{16 \pi \alpha\left|\lambda_{L}^{i} \lambda_{L}^{j}\right|^{2}}{m_{\chi}^{2} s_{W}^{2}} f_{W}^{F_{r}}(x, y), \\
& \left|\mathcal{M}_{h}^{F_{r}}\right|^{2}=\frac{1}{2}\left|\mathcal{M}_{h}^{S_{c}}\right|^{2}
\end{aligned}
$$

where

$$
f_{V}^{F_{r}}(x, y)=\frac{\left(1-x+\frac{\xi_{V}}{4}\right)\left(2-2 x+x^{2}-4 y+2 x y+2 y^{2}-\frac{\xi_{V}}{2}\right)}{(1-\mu-2 y)^{2}(3+\mu-2 x-2 y)^{2}} .
$$

Differential cross sections:

$$
\begin{aligned}
\frac{d(\sigma v)_{\ell^{i} \bar{\ell}^{j} Z}^{F_{r}}}{d x} & =\frac{\alpha\left|\lambda_{L}^{i} \lambda_{L}^{j}\right|^{2}}{32 \pi^{2} m_{\chi}^{2}} \frac{\left(\left(T_{3}\right)_{\ell_{L}}-Q_{\ell} s_{W}^{2}\right)^{2}}{c_{W}^{2} s_{W}^{2}} g_{Z}^{F_{r}}(x), \\
\frac{d(\sigma v)_{\ell^{i} \bar{\ell}^{j} W}^{F_{r_{r}}}}{d x} & =\frac{\alpha\left|\lambda_{L}^{i} \lambda_{L}^{j}\right|^{2}}{32 \pi^{2} m_{\chi}^{2} s_{W}^{2}} g_{W}^{F_{r}}(x),
\end{aligned}
$$

where

$$
\begin{aligned}
g_{V}^{F_{r}}(x)= & \left(1-x+\frac{\xi_{V}}{4}\right)\left[\frac{2 \sqrt{x^{2}-\xi_{Z}}}{(1+\mu)(1+\mu-2 x)+\xi_{Z}}-\frac{\sqrt{x^{2}-\xi_{Z}}}{(1+\mu-x)^{2}}\right. \\
& \left.-\frac{(1+\mu)(1+\mu-2 x)+\xi_{Z}}{(1+\mu-x)^{3}} \tanh ^{-1}\left(\frac{\sqrt{x^{2}-\xi_{Z}}}{1+\mu-x}\right)\right] .
\end{aligned}
$$


3. $\chi \chi \rightarrow V V^{\prime}$.

$$
\begin{aligned}
(\sigma v)_{\gamma \gamma}^{F_{r}} & =\frac{\alpha^{2} Q_{e}^{2}}{256 \pi^{3} m_{\chi}^{2}}\left(\sum_{i}\left|\lambda_{L}^{i}\right|^{2}\right)^{2}\left[\operatorname{Li}_{2}\left(\frac{1}{\mu}\right)-\operatorname{Li}_{2}\left(-\frac{1}{\mu}\right)\right]^{2} \\
(\sigma v)_{\gamma Z}^{F_{r}} & =\frac{\alpha^{2} Q_{e}^{2}\left(\left(T_{3}\right)_{e_{L}}-Q_{e} s_{W}^{2}\right)^{2}}{512 \pi^{3} m_{\chi}^{2} c_{W}^{2} s_{W}^{2}}\left(\sum_{i}\left|\lambda_{L}^{i}\right|^{2}\right)^{2} \frac{\left|A_{\gamma Z}^{F}\right|^{2}}{\mu^{2}\left(1-\frac{\xi_{Z}}{4}\right)\left(1-\frac{\xi_{Z}^{2}}{16 \mu^{2}}\right)^{2}} \\
(\sigma v)_{Z Z}^{F_{r}} & =\frac{\alpha^{2}}{1024 \pi^{3} m_{\chi}^{2} c_{W}^{4} s_{W}^{4}}\left(\sum_{i} \sum_{\ell=e, \nu_{L}}\left|\lambda_{L}^{i}\right|^{2}\left(\left(T_{3}\right)_{\ell_{L}}-Q_{\ell} s_{W}^{2}\right)^{2}\right)^{2} \frac{\left|A_{Z Z}^{F}\right|^{2}}{\mu^{2} \sqrt{1-\xi_{Z}}} \\
(\sigma v)_{W W}^{F_{r}} & =\frac{\alpha^{2}}{2048 \pi^{3} m_{\chi}^{2} s_{W}^{4}}\left(\sum_{i}\left|\lambda_{L}^{i}\right|^{2}\right)^{2} \frac{\left|A_{W W}^{F}\right|^{2}}{\mu^{2} \sqrt{1-\xi_{W}}}
\end{aligned}
$$

where $A_{\gamma Z}^{F}, A_{Z Z}^{F}$ and $A_{W W}^{F}$ are

$$
\begin{aligned}
A_{\gamma Z}^{F}= & m_{\chi}^{2}\left(1-\frac{\xi_{Z}}{4 \mu}\right)\left\{2 \mu\left(1-\frac{\xi_{Z}}{4}\right)^{2} C_{0}\left(m_{\chi}^{2}, 0, \frac{m_{Z}^{2}}{2}-m_{\chi}^{2} ; 0, m_{\widetilde{L}}^{2}, m_{\widetilde{L}}^{2}\right)\right. \\
& +\xi_{Z}\left(\frac{1+\mu^{2}}{2}+\frac{\xi_{Z}(\mu-1)}{4}+\frac{\xi_{Z}^{2}}{16}\right) C_{0}\left(m_{\chi}^{2}, m_{Z}^{2}, \frac{m_{Z}^{2}}{2}-m_{\chi}^{2} ; m_{\widetilde{L}}^{2}, 0,0\right) \\
& \left.+\left(\mu+\frac{\xi_{Z}}{4}\right)\left(2-\xi_{Z}+\frac{\mu \xi_{Z}}{2}\right) C_{0}\left(m_{\chi}^{2}, m_{Z}^{2}, \frac{m_{Z}^{2}}{2}-m_{\chi}^{2} ; 0, m_{\widetilde{L}}^{2}, m_{\widetilde{L}}^{2}\right)\right\} \\
& +\frac{\mu \xi_{Z}}{2}\left(1-\frac{\xi_{Z}^{2}}{16 \mu^{2}}\right)\left[2 \sqrt{\frac{4 \mu}{\xi_{Z}}-1} \operatorname{arccot}\left(\sqrt{\frac{4 \mu}{\xi_{Z}}-1}\right)-\log \left(\frac{\xi_{Z}}{\mu}\right)+i \pi\right],
\end{aligned}
$$

and

$$
\begin{aligned}
A_{Z Z}^{F}= & m_{\chi}^{2}\left\{\xi_{Z}\left(1+\mu^{2}-\xi_{Z}\right) C_{0}\left(m_{\chi}^{2}, m_{Z}^{2}, m_{Z}^{2}-m_{\chi}^{2} ; m_{\widetilde{L}}^{2}, 0,0\right)\right. \\
& \left.+\left[4 \mu+\left(\mu^{2}-4 \mu-1\right) \xi_{Z}+\xi_{Z}^{2}\right] C_{0}\left(m_{\chi}^{2}, m_{Z}^{2}, m_{Z}^{2}-m_{\chi}^{2} ; 0, m_{\widetilde{L}}^{2}, m_{\widetilde{L}}^{2}\right)\right\} \\
& +\mu \xi_{Z}\left[2 \sqrt{\frac{4 \mu}{\xi_{Z}}-1} \operatorname{arccot}\left(\sqrt{\frac{4 \mu}{\xi_{Z}}-1}\right)-\log \left(\frac{\xi_{Z}}{\mu}\right)+i \pi\right] \\
A_{W W}^{F}= & \left.A_{Z Z}^{F}\right|_{m_{Z} \rightarrow m_{W}} .
\end{aligned}
$$

\section{A.4.2 Direct detection}

In Majorana case, $a_{\chi}, C_{A, q}^{Z}$ and $C_{S, q}^{\text {Higgs }}$ are non-vanishing. With the notation of eq. (A.33), the coefficients are exactly same as the Dirac case.

\section{B Direct detection limit with non-contact interactions}

In this appendix, we briefly explain how we have calculated the number of DM-nuclei scattering events in presence of non-contact interactions, and recast the public limit [107], that assumes contract-type DM-nucleon interactions, to our cases. Dedicated studies of this subject can be found in the context of mutipolar dark matter [113-118], where they 
make some likelihood analysis to calculate the number of events. In this paper, we have performed simpler analysis.

The total expected scattering rate (per target mass) is expressed in terms of integrals of the differential rate over nuclear recoil energy $E_{R}$ :

$$
R=\int_{E_{R, \mathrm{th}}}^{\infty} d E_{R} \epsilon\left(E_{R}\right) \sum_{i} \eta_{i} \frac{d R_{i}}{d E_{R}}
$$

where $\eta_{i}$ is an isotope fraction of target nuclei (see e.g. ref. [119]) and $\epsilon\left(E_{R}\right)$ an efficiency function for a given recoil energy $E_{R}$, for which we used the black solid line in figure 1 of [107], which is a best-fit total efficiency after taking the energy region-of-interest (ROI) selection into account. The differential scattering rate (per target mass) is given by

$$
\frac{d R_{i}}{d E_{R}}=\frac{\rho_{0}}{m_{\chi} m_{N_{i}}} \int_{|\vec{v}|>v_{\min }\left(E_{R}\right)} d^{3} \vec{v} v f_{E}(\vec{v}) \frac{d \sigma_{i}\left(v, E_{R}\right)}{d E_{R}},
$$

where $v=|\vec{v}|$ and $v_{\text {min }}=\sqrt{m_{N} E_{R} /\left(2 \mu_{\text {red }}^{2}\right)}$ is the minimum velocity with $\mu_{\text {red }}$ the DMnucleus reduced mass. The differential cross section for an elastic DM-nucleus scattering, $d \sigma / d E_{R}$, is given by eq. (4.13), which involves two nuclear form factors $F\left(E_{R}\right)$ and $F_{\text {spin }}\left(E_{R}\right)$, for which we use the Helm form factor normalized to $F(0)=1$, and a spin form factor in [106] with a thin-shell approximation, respectively. The DM velocity distribution in the earth frame $f_{E}(\vec{v})$ is obtained by the Galilean transformation of a velocity distribution in the galactic rest frame $f_{G}(\vec{v})$, for which we use an isotropic Maxwellian distribution with a smooth cutoff [101, 120-123],

$$
f_{G}(\vec{v})=\frac{N}{\left(v_{0} \sqrt{\pi}\right)^{3}}\left(e^{-\vec{v}^{2} / v_{0}^{2}}-e^{-v_{\mathrm{esc}}^{2} / v_{0}^{2}}\right) \theta\left(v_{\mathrm{esc}}-v\right)
$$

where $N$ is a normalization constant such that $\int d^{3} \vec{v} f_{G}(\vec{v})=1$. In our analysis, we take $\rho_{0}=0.3 \mathrm{GeV} / \mathrm{cm}^{3}, v_{0}=220 \mathrm{~km} / \mathrm{s}$ and $v_{\text {esc }}=544 \mathrm{~km} / \mathrm{s}[122,123]$.

Combining all pieces above, we can calculate the expected number of DM-nuclei scattering events detected at experiments, given masses of DM and vectorlike lepton and Yukawa coupling. Then, we can translate the null results at direct detection into limits on our models by comparing our calculation with experimental results at scattering event level. To pull the number of exclusion events out of the public limit (the solid black line in figure 5 of [107]), we required that contact-type scattering reproduces the exclusion curve. The number of exclusion events extracted in this way is $N_{\text {exc. }} \simeq 5.6,12,14$ for $m_{\mathrm{DM}}=30 \mathrm{GeV}, 100 \mathrm{GeV}, 1 \mathrm{TeV}$, respectively. This is the way we have drawn the red lines in figures 7 and 8 in the text. We have confirmed that our approach produces consistent results with [21] which studied the same type of DM candidate before. ${ }^{6}$

\footnotetext{
${ }^{6}$ The definition of our signal region differs by a factor 2 from the definition in ref. [21], in which the authors defined their signal region as the lower half of the mean nuclear recoil band (e.g. the red solid line in figure 4 of [124]). Thus, our bound is aggressive by the factor. Once we use their definition instead of ours, we can find practically the same exclusion line as what they gave in [21].
} 
We would like to note that even if we take statistical methods more carefully, the results are not affected so much. Taking the statistical fluctuations into account, the number of DM-nuclei scattering events is expressed by

$$
\begin{aligned}
R= & \int_{S_{1}^{\min }}^{S_{1}^{\max }} d S_{1} \sum_{n=0}^{\infty} \operatorname{Gauss}\left(S_{1} \mid n, \sqrt{n} \sigma_{\mathrm{PMT}}\right) \\
& \times \int_{E_{\min }}^{\infty} d E_{R} \tilde{\epsilon}\left(E_{R}\right) \operatorname{Poiss}\left(n \mid \nu\left(E_{R}\right)\right) \sum_{i} \frac{d R_{i}}{d E_{R}},
\end{aligned}
$$

with $\tilde{\epsilon}\left(E_{R}\right)$ an efficiency function, ${ }^{7}$ and $\nu\left(E_{R}\right)$ the expected number of photoelectrons (PEs) for a given recoil energy $E_{R}$, for which, respectively, we can take the green line in figure 1 of [107] and the black dotted line in figure 13 of [111]. The PMT efficiency can be taken to $\sigma_{\mathrm{PMT}}=0.4[125,126]$, for example. With this expression and using $S_{1}^{\min }=3 \mathrm{PEs}$ and $S_{1}^{\max }=70$ PEs, we would be able to calculate the expected number of scattering events in the parameter space considered. We have checked that, for some reference points, this statistical treatment gives practically the same results as those of our simpler analysis.

\section{Renormalization group equations}

\section{C.1 Scalar DM}

\section{C.1.1 Complex scalar DM}

The Yukawa interaction is given by

$$
-\mathcal{L}=Y_{e}^{i j} \bar{\ell}_{L}^{i} H e_{R}^{j}+\kappa \bar{L}_{L} H E_{R}+\tilde{\kappa} \bar{E}_{L} \tilde{H} L_{R}+\lambda_{L}^{i} \bar{\ell}_{L}^{i} X L_{R}+\lambda_{R}^{i} \bar{E}_{L} X^{*} e_{R}^{i}+\text { h.c.. }
$$

The RGEs of the Yukawa couplings are given by

$$
\begin{aligned}
16 \pi^{2} \beta_{\lambda_{L}^{i}}= & 2 \tilde{\kappa} Y_{e}^{i j} \lambda_{R}^{* j}+\lambda_{L}^{i} \lambda_{L}^{* j} \lambda_{L}^{j}+\frac{1}{2}\left(Y_{e} Y_{e}^{\dagger}\right)^{i j} \lambda_{L}^{j}+\lambda_{L}^{i}\left(\frac{1}{2} \tilde{\kappa}^{*} \tilde{\kappa}+Y_{2}(X)-\frac{9}{4}\left(g_{1}^{2}+g_{2}^{2}\right)\right), \\
16 \pi^{2} \beta_{\lambda_{R}^{i}}= & 4 \tilde{\kappa} \lambda_{L}^{* j} Y_{e}^{j i}+\lambda_{R}^{j}\left(Y_{e}^{\dagger} Y_{e}\right)^{j i}+\lambda_{R}^{i}\left(\lambda_{R}^{* j} \lambda_{R}^{j}+\tilde{\kappa}^{*} \tilde{\kappa}+Y_{2}(X)-\frac{9}{4}\left(g_{1}^{2}+g_{2}^{2}\right)\right), \quad(\mathrm{C} .3) \\
16 \pi^{2} \beta_{\kappa}= & \kappa\left(\frac{3}{2} \kappa^{*} \kappa+Y_{2}(H)-\frac{9}{4}\left(g_{1}^{2}+g_{2}^{2}\right)\right), \\
16 \pi^{2} \beta_{\tilde{\kappa}}= & 2 \lambda_{R}^{i}\left(Y_{e}^{\dagger}\right)_{i j} \lambda_{L}^{j}+\tilde{\kappa}\left(\frac{3}{2} \tilde{\kappa}^{*} \tilde{\kappa}+\frac{1}{2} \lambda_{L}^{* i} \lambda_{L}^{i}+\frac{1}{2} \lambda_{R}^{* i} \lambda_{R}^{i}+Y_{2}(H)-\frac{9}{4}\left(g_{1}^{2}+g_{2}^{2}\right)\right), \\
16 \pi^{2} \beta_{Y_{e}^{i j}}= & \frac{3}{2}\left(Y_{e} Y_{e}^{\dagger} Y_{e}\right)^{i j}+2 \tilde{\kappa} \lambda_{L}^{i} \lambda_{R}^{j}+\frac{1}{2} \tilde{\kappa}\left(\lambda_{L}^{i}\left(\lambda_{L}^{\dagger} Y_{e}\right)^{j}+\left(Y_{e} \lambda_{R}^{\dagger}\right)^{i} \lambda_{R}^{j}\right) \\
& +Y_{e}^{i j}\left(Y_{2}(H)-\frac{9}{4}\left(g_{1}^{2}+g_{2}^{2}\right)\right),
\end{aligned}
$$

\footnotetext{
${ }^{7}$ This is a different function from eq. (B.1).
} 
where

$$
\begin{aligned}
& Y_{2}(X)=2 \lambda_{L}^{* i} \lambda_{L}^{i}+\lambda_{R}^{* i} \lambda_{R}^{i}, \\
& Y_{2}(H)=\operatorname{Tr}\left(3 Y_{u} Y_{u}^{\dagger}+3 Y_{d} Y_{d}^{\dagger}+Y_{e} Y_{e}^{\dagger}\right)+\kappa^{*} \kappa+\tilde{\kappa}^{*} \tilde{\kappa} .
\end{aligned}
$$

Here, $Y_{u}$ and $Y_{d}$ are the up and down quark Yukawa matrices in the SM, respectively. We show the full 1-loop RGEs for completeness. We neglect the Yukawa couplings except $\lambda_{L}^{2}$ and $\lambda_{R}^{2}$ in our numerical evaluation. Note that sizable $\kappa$ and/or $\tilde{\kappa}$ will make the Landau pole scale lower.

\section{C.1.2 Real scalar DM}

The interactions are

$$
-\mathcal{L}=Y_{e}^{i j} \bar{\ell}_{L}^{i} H e_{R}^{j}+\kappa \bar{L}_{L} H E_{R}+\tilde{\kappa} \bar{E}_{L} \tilde{H} L_{R}+\lambda_{L}^{i} \bar{\ell}_{L}^{i} X L_{R}+\lambda_{R}^{i} \bar{E}_{L} X e_{R}^{i}+\text { h.c. },
$$

where $X$ is now a real scalar field.

Compared with the complex scalar DM case, there are Yukawa coupling renormalization fro $\lambda_{L}, \lambda_{R}$,

$$
\begin{aligned}
& 16 \pi^{2} \beta_{\lambda_{L}^{i}}=2 \tilde{\kappa} Y_{e}^{i j} \lambda_{R}^{* j}+3 \lambda_{L}^{i} \lambda_{L}^{* j} \lambda_{L}^{j}+\frac{1}{2}\left(Y_{e} Y_{e}^{\dagger}\right)^{i j} \lambda_{L}^{j}+\lambda_{L}^{i}\left(\frac{1}{2} \tilde{\kappa}^{*} \tilde{\kappa}+Y_{2}(X)-\frac{9}{4}\left(g_{1}^{2}+g_{2}^{2}\right)\right), \\
& 16 \pi^{2} \beta_{\lambda_{R}^{i}}=4 \tilde{\kappa} \lambda_{L}^{* j} Y_{e}^{j i}+\lambda_{R}^{j}\left(Y_{e}^{\dagger} Y_{e}\right)^{j i}+\lambda_{R}^{i}\left(3 \lambda_{R}^{* j} \lambda_{R}^{j}+\tilde{\kappa}^{*} \tilde{\kappa}+Y_{2}(X)-\frac{9}{4}\left(g_{1}^{2}+g_{2}^{2}\right)\right),
\end{aligned}
$$

and the other parts are same as the complex case.

\section{C.2 Fermion DM}

The Yukawa interactions are

$$
-\mathcal{L}=\lambda_{L}^{i} \bar{\ell}_{L}^{i} \chi \tilde{L}_{R}+\lambda_{R}^{i} \tilde{E}_{L}^{*} \bar{\chi} e_{R}^{i}+\text { h.c. },
$$

where the SM Yukawa matrix $Y_{e}$ is neglected.

\section{C.2.1 Dirac fermion}

The RGEs are given by

$$
\begin{aligned}
16 \pi^{2} \beta_{\lambda_{L}^{i}} & =\lambda_{L}^{i}\left[\frac{5}{2}\left(\lambda_{L}^{\dagger} \lambda_{L}\right)+\frac{1}{2}\left(\lambda_{R}^{\dagger} \lambda_{R}\right)-\frac{9}{20} g_{1}^{2}-\frac{9}{4} g_{2}^{2}\right] \\
16 \pi^{2} \beta_{\lambda_{R}^{i}} & =\lambda_{R}^{i}\left[2\left(\lambda_{R}^{\dagger} \lambda_{R}\right)+2\left(\lambda_{L}^{\dagger} \lambda_{L}\right)-\frac{9}{5} g_{1}^{2}\right] .
\end{aligned}
$$

\section{C.2.2 Majorana fermion}

The RGEs are given by

$$
\begin{aligned}
16 \pi^{2} \beta_{\lambda_{L}^{i}} & =\frac{1}{2}\left(Y_{e} Y_{e}^{\dagger} \lambda_{L}\right)^{i}+\lambda_{L}^{i}\left[\frac{9}{2}\left(\lambda_{L}^{\dagger} \lambda_{L}\right)+\frac{1}{2}\left(\lambda_{R}^{\dagger} \lambda_{R}\right)-\frac{9}{20} g_{1}^{2}-\frac{9}{4} g_{2}^{2}\right], \\
16 \pi^{2} \beta_{\lambda_{R}^{i}} & =\frac{1}{2}\left(\lambda_{R} Y_{e}^{\dagger} Y_{e}\right)^{i}+\lambda_{R}^{i}\left[4\left(\lambda_{R}^{\dagger} \lambda_{R}\right)+2\left(\lambda_{L}^{\dagger} \lambda_{L}\right)-\frac{9}{5} g_{1}^{2}\right] .
\end{aligned}
$$


Open Access. This article is distributed under the terms of the Creative Commons Attribution License (CC-BY 4.0), which permits any use, distribution and reproduction in any medium, provided the original author(s) and source are credited.

\section{References}

[1] G. Jungman, M. Kamionkowski and K. Griest, Supersymmetric dark matter, Phys. Rept. 267 (1996) 195 [hep-ph/9506380] [INSPIRE].

[2] J.R. Ellis, K.A. Olive, Y. Santoso and V.C. Spanos, Supersymmetric dark matter in light of WMAP, Phys. Lett. B 565 (2003) 176 [hep-ph/0303043] [INSPIRE].

[3] G. Servant and T.M.P. Tait, Is the lightest Kaluza-Klein particle a viable dark matter candidate?, Nucl. Phys. B 650 (2003) 391 [hep-ph/0206071] [INSPIRE].

[4] H.-C. Cheng, J.L. Feng and K.T. Matchev, Kaluza-Klein dark matter, Phys. Rev. Lett. 89 (2002) 211301 [hep-ph/0207125] [INSPIRE].

[5] A. Arhrib, Y.-L.S. Tsai, Q. Yuan and T.-C. Yuan, An updated analysis of inert Higgs doublet model in light of the recent results from LUX, PLANCK, AMS-02 and LHC, JCAP 06 (2014) 030 [arXiv:1310.0358] [INSPIRE].

[6] A. Goudelis, B. Herrmann and O. Stål, Dark matter in the inert doublet model after the discovery of a Higgs-like boson at the LHC, JHEP 09 (2013) 106 [arXiv:1303.3010] [INSPIRE].

[7] A. Ilnicka, M. Krawczyk and T. Robens, Inert doublet model in light of LHC Run I and astrophysical data, Phys. Rev. D 93 (2016) 055026 [arXiv:1508.01671] [inSPIRE].

[8] M.A. Díaz, B. Koch and S. Urrutia-Quiroga, Constraints to dark matter from inert Higgs doublet model, Adv. High Energy Phys. 2016 (2016) 8278375 [arXiv:1511. 04429] [INSPIRE].

[9] A. Belyaev, G. Cacciapaglia, I.P. Ivanov, F. Rojas-Abatte and M. Thomas, Anatomy of the inert two Higgs doublet model in the light of the LHC and non-LHC dark matter searches, Phys. Rev. D 97 (2018) 035011 [arXiv:1612.00511] [InSPIRE].

[10] S. Kanemura, S. Matsumoto, T. Nabeshima and N. Okada, Can WIMP dark matter overcome the nightmare scenario?, Phys. Rev. D 82 (2010) 055026 [arXiv:1005.5651] [INSPIRE].

[11] A. Djouadi, O. Lebedev, Y. Mambrini and J. Quevillon, Implications of LHC searches for Higgs-portal dark matter, Phys. Lett. B 709 (2012) 65 [arXiv:1112.3299] [INSPIRE].

[12] A. Djouadi, A. Falkowski, Y. Mambrini and J. Quevillon, Direct detection of Higgs-portal dark matter at the LHC, Eur. Phys. J. C 73 (2013) 2455 [arXiv:1205.3169] [InSPIRE].

[13] M. Escudero, A. Berlin, D. Hooper and M.-X. Lin, Toward (finally!) ruling out $Z$ and Higgs mediated dark matter models, JCAP 12 (2016) 029 [arXiv: 1609.09079] [INSPIRE].

[14] J. Ellis, A. Fowlie, L. Marzola and M. Raidal, Statistical analyses of Higgs- and Z-portal dark matter models, Phys. Rev. D 97 (2018) 115014 [arXiv:1711.09912] [INSPIRE].

[15] P. Athron, J.M. Cornell, F. Kahlhoefer, J. McKay, P. Scott and S. Wild, Impact of vacuum stability, perturbativity and XENON1T on global fits of $\mathbb{Z}_{2}$ and $\mathbb{Z}_{3}$ scalar singlet dark matter, Eur. Phys. J. C 78 (2018) 830 [arXiv:1806.11281] [INSPIRE]. 
[16] S. Chang, R. Edezhath, J. Hutchinson and M. Luty, Effective WIMPs, Phys. Rev. D 89 (2014) 015011 [arXiv: 1307.8120] [INSPIRE].

[17] Y. Bai and J. Berger, Fermion portal dark matter, JHEP 11 (2013) 171 [arXiv:1308.0612] [INSPIRE].

[18] Y. Bai and J. Berger, Lepton portal dark matter, JHEP 08 (2014) 153 [arXiv:1402.6696] [INSPIRE].

[19] S. Chang, R. Edezhath, J. Hutchinson and M. Luty, Leptophilic effective WIMPs, Phys. Rev. D 90 (2014) 015011 [arXiv:1402.7358] [INSPIRE].

[20] M. Garny, A. Ibarra, S. Rydbeck and S. Vogl, Majorana dark matter with a coloured mediator: collider vs. direct and indirect searches, JHEP 06 (2014) 169 [arXiv:1403.4634] [INSPIRE].

[21] A. Ibarra and S. Wild, Dirac dark matter with a charged mediator: a comprehensive one-loop analysis of the direct detection phenomenology, JCAP 05 (2015) 047 [arXiv:1503.03382] [INSPIRE].

[22] B. Bhattacharya, D. London, J.M. Cline, A. Datta and G. Dupuis, Quark-flavored scalar dark matter, Phys. Rev. D 92 (2015) 115012 [arXiv:1509.04271] [INSPIRE].

[23] T. Abe, J. Kawamura, S. Okawa and Y. Omura, Dark matter physics, flavor physics and LHC constraints in the dark matter model with a bottom partner, JHEP 03 (2017) 058 [arXiv: 1612.01643] [INSPIRE].

[24] S. Baek, P. Ko and P. Wu, Top-philic scalar dark matter with a vector-like fermionic top partner, JHEP 10 (2016) 117 [arXiv:1606.00072] [INSPIRE].

[25] S. Baek, P. Ko and P. Wu, Heavy quark-philic scalar dark matter with a vector-like fermion portal, JCAP 07 (2018) 008 [arXiv:1709.00697] [INSPIRE].

[26] M. Blanke and S. Kast, Top-flavoured dark matter in dark minimal flavour violation, JHEP 05 (2017) 162 [arXiv: 1702.08457] [INSPIRE].

[27] J. Kawamura, S. Okawa and Y. Omura, Interplay between the $b \rightarrow$ sll anomalies and dark matter physics, Phys. Rev. D 96 (2017) 075041 [arXiv: 1706.04344] [InSPIRE].

[28] S. Colucci, B. Fuks, F. Giacchino, L. Lopez Honorez, M.H.G. Tytgat and J. Vandecasteele, Top-philic vector-like portal to scalar dark matter, Phys. Rev. D 98 (2018) 035002 [arXiv: 1804.05068] [INSPIRE].

[29] J. Kawamura, S. Okawa, Y. Omura and Y. Tang, WIMP dark matter in the parity solution to the strong CP problem, JHEP 04 (2019) 162 [arXiv: 1812.07004] [INSPIRE].

[30] Particle Data Group collaboration, Review of particle physics, Phys. Rev. D 98 (2018) 030001 [INSPIRE].

[31] M. Davier, A. Hoecker, B. Malaescu and Z. Zhang, A new evaluation of the hadronic vacuum polarisation contributions to the muon anomalous magnetic moment and to $\alpha\left(m_{Z}^{2}\right)$, Eur. Phys. J. C 80 (2020) 241 [Erratum ibid. 80 (2020) 410] [arXiv:1908.00921] [INSPIRE].

[32] A. Keshavarzi, D. Nomura and T. Teubner, $g-2$ of charged leptons, $\alpha\left(M_{Z}^{2}\right)$ and the hyperfine splitting of muonium, Phys. Rev. D 101 (2020) 014029 [arXiv:1911.00367] [INSPIRE]. 
[33] RBC, UKQCD collaboration, Calculation of the hadronic vacuum polarization contribution to the muon anomalous magnetic moment, Phys. Rev. Lett. 121 (2018) 022003 [arXiv: 1801.07224] [INSPIRE].

[34] A. Gérardin et al., The leading hadronic contribution to $(g-2)_{\mu}$ from lattice $Q C D$ with $N_{\mathrm{f}}=2+1$ flavours of $O(a)$ improved Wilson quarks, Phys. Rev. D 100 (2019) 014510 [arXiv: 1904.03120] [INSPIRE].

[35] Fermilab Lattice, LATTICE-HPQCD, MILC collaboration, Hadronic-vacuum-polarization contribution to the muon's anomalous magnetic moment from four-flavor lattice QCD, Phys. Rev. D 101 (2020) 034512 [arXiv:1902.04223] [InSPIRE].

[36] D. Giusti, V. Lubicz, G. Martinelli, F. Sanfilippo and S. Simula, Electromagnetic and strong isospin-breaking corrections to the muon $g-2$ from Lattice $Q C D+Q E D$, Phys. Rev. D 99 (2019) 114502 [arXiv: 1901.10462] [INSPIRE].

[37] S. Borsányi et al., Leading-order hadronic vacuum polarization contribution to the muon magnetic momentfrom lattice QCD, arXiv:2002.12347 [INSPIRE].

[38] T. Blum et al., Hadronic light-by-light scattering contribution to the muon anomalous magnetic moment from lattice QCD, Phys. Rev. Lett. 124 (2020) 132002 [arXiv: 1911.08123] [INSPIRE].

[39] A. Gérardin et al., Hadronic light-by-light scattering amplitudes from lattice QCD versus dispersive sum rules, Phys. Rev. D 98 (2018) 074501 [arXiv:1712.00421] [INSPIRE].

[40] J. Green, O. Gryniuk, G. von Hippel, H.B. Meyer and V. Pascalutsa, Lattice QCD calculation of hadronic light-by-light scattering, Phys. Rev. Lett. 115 (2015) 222003 [arXiv: 1507.01577] [INSPIRE].

[41] H.B. Meyer and H. Wittig, Lattice QCD and the anomalous magnetic moment of the muon, Prog. Part. Nucl. Phys. 104 (2019) 46 [arXiv:1807.09370] [InSPIRE].

[42] F. Campanario et al., Standard model radiative corrections in the pion form factor measurements do not explain the $a_{\mu}$ anomaly, Phys. Rev. D 100 (2019) 076004 [arXiv: 1903.10197] [INSPIRE].

[43] J. Kopp, L. Michaels and J. Smirnov, Loopy constraints on leptophilic dark matter and internal bremsstrahlung, JCAP 04 (2014) 022 [arXiv:1401.6457] [INSPIRE].

[44] P. Agrawal, Z. Chacko and C.B. Verhaaren, Leptophilic dark matter and the anomalous magnetic moment of the muon, JHEP 08 (2014) 147 [arXiv: 1402.7369] [INSPIRE].

[45] K. Kowalska and E.M. Sessolo, Expectations for the muon $g-2$ in simplified models with dark matter, JHEP 09 (2017) 112 [arXiv:1707.00753] [INSPIRE].

[46] L. Calibbi, R. Ziegler and J. Zupan, Minimal models for dark matter and the muon $g-2$ anomaly, JHEP 07 (2018) 046 [arXiv: 1804.00009] [INSPIRE].

[47] K. Fukushima and J. Kumar, Dipole moment bounds on dark matter annihilation, Phys. Rev. D 88 (2013) 056017 [arXiv: 1307.7120] [INSPIRE].

[48] R. Dermisek and A. Raval, Explanation of the muon $g-2$ anomaly with vectorlike leptons and its implications for Higgs decays, Phys. Rev. D 88 (2013) 013017 [arXiv:1305.3522] [INSPIRE].

[49] F. Jegerlehner and A. Nyffeler, The muon g-2, Phys. Rept. 477 (2009) 1 [arXiv: 0902 .3360] [INSPIRE]. 
[50] K.R. Lynch, A note on one loop electroweak contributions to $g-2$ : a companion to BUHEP-01-16, hep-ph/0108081 [INSPIRE].

[51] H. Davoudiasl and W.J. Marciano, Tale of two anomalies, Phys. Rev. D 98 (2018) 075011 [arXiv: 1806.10252] [INSPIRE].

[52] J. Kawamura, S. Raby and A. Trautner, Complete vectorlike fourth family and new U(1)' for muon anomalies,x Phys. Rev. D 100 (2019) 055030 [arXiv:1906.11297] [INSPIRE].

[53] A.E. Cárcamo Hernández, S.F. King, H. Lee and S.J. Rowley, Is it possible to explain the muon and electron $g-2$ in a $Z$ ' model?, Phys. Rev. D 101 (2020) 11 [arXiv:1910.10734] [INSPIRE].

[54] J. Kawamura, S. Raby and A. Trautner, Complete vectorlike fourth family with U(1)': a global analysis, Phys. Rev. D 101 (2020) 035026 [arXiv:1911.11075] [INSPIRE].

[55] G.F. Giudice, P. Paradisi and M. Passera, Testing new physics with the electron $g-2$, JHEP 11 (2012) 113 [arXiv:1208.6583] [InSPIRE].

[56] A. Crivellin, M. Hoferichter and P. Schmidt-Wellenburg, Combined explanations of $(g-2) \_\mu, e$ and implications for a large muon EDM, Phys. Rev. D 98 (2018) 113002 [arXiv: 1807.11484] [INSPIRE].

[57] J. Liu, C.E.M. Wagner and X.-P. Wang, A light complex scalar for the electron and muon anomalous magnetic moments, JHEP 03 (2019) 008 [arXiv:1810.11028] [INSPIRE].

[58] B. Dutta and Y. Mimura, Electron g-2 with flavor violation in MSSM, Phys. Lett. B 790 (2019) 563 [arXiv: 1811.10209] [INSPIRE].

[59] R.H. Parker, C. Yu, W. Zhong, B. Estey and H. Müller, Measurement of the fine-structure constant as a test of the Standard Model, Science 360 (2018) 191 [arXiv:1812.04130] [INSPIRE].

[60] X.-F. Han, T. Li, L. Wang and Y. Zhang, Simple interpretations of lepton anomalies in the lepton-specific inert two-Higgs-doublet model, Phys. Rev. D 99 (2019) 095034 [arXiv: 1812.02449] [INSPIRE].

[61] M. Endo and W. Yin, Explaining electron and muon $g-2$ anomaly in SUSY without lepton-flavor mixings, JHEP 08 (2019) 122 [arXiv:1906.08768] [INSPIRE].

[62] M. Abdullah, B. Dutta, S. Ghosh and T. Li, $(g-2)_{\mu, e}$ and the ANITA anomalous events in a three-loop neutrino mass model, Phys. Rev. D 100 (2019) 115006 [arXiv:1907.08109] [INSPIRE].

[63] M. Bauer, M. Neubert, S. Renner, M. Schnubel and A. Thamm, Axion-like particles, lepton-flavor violation and a new explanation of $a_{\mu}$ and $a_{e}$, Phys. Rev. Lett. 124 (2020) 211803 [arXiv: 1908.00008] [INSPIRE].

[64] C. Cornella, P. Paradisi and O. Sumensari, Hunting for ALPs with lepton flavor violation, JHEP 01 (2020) 158 [arXiv:1911.06279] [INSPIRE].

[65] ATLAS collaboration, Search for direct stau production in events with two hadronic $\tau$-leptons in $\sqrt{s}=13 \mathrm{TeV}$ pp collisions with the ATLAS detector, Phys. Rev. D 101 (2020) 032009 [arXiv: 1911.06660] [INSPIRE].

[66] ATLAS collaboration, Searches for electroweak production of supersymmetric particles with compressed mass spectra in $\sqrt{s}=13$ TeV pp collisions with the ATLAS detector, Phys. Rev. D 101 (2020) 052005 [arXiv: 1911.12606] [INSPIRE]. 
[67] ATLAS collaboration, Search for electroweak production of charginos and sleptons decaying into final states with two leptons and missing transverse momentum in $\sqrt{s}=13 \mathrm{TeV} p p$ collisions using the ATLAS detector, Eur. Phys. J. C 80 (2020) 123 [arXiv:1908.08215] [INSPIRE].

[68] CMS collaboration, Search for supersymmetry in events with $\tau$ leptons and missing transverse momentum in proton-proton collisions at $\sqrt{s}=13 \mathrm{TeV}$, JHEP 11 (2018) 151 [arXiv: 1807.02048].

[69] CMS collaboration, Search for supersymmetric partners of electrons and muons in proton-proton collisions at $\sqrt{s}=13$ TeV, Phys. Lett. B 790 (2019) 140 [arXiv: 1806.05264] [INSPIRE].

[70] CMS collaboration, Search for direct $\tau$ slepton pair production in proton-proton collisions at $\sqrt{s}=13 \mathrm{TeV}$, CMS-PAS-SUS-18-006 (2018).

[71] G. Bozzi, B. Fuks and M. Klasen, Threshold resummation for slepton-pair production at hadron colliders, Nucl. Phys. B 777 (2007) 157 [hep-ph/0701202] [INSPIRE].

[72] B. Fuks, M. Klasen, D.R. Lamprea and M. Rothering, Precision predictions for electroweak superpartner production at hadron colliders with Resummino, Eur. Phys. J. C 73 (2013) 2480 [arXiv: 1304.0790] [INSPIRE].

[73] B. Fuks, M. Klasen, D.R. Lamprea and M. Rothering, Revisiting slepton pair production at the Large Hadron Collider, JHEP 01 (2014) 168 [arXiv: 1310.2621] [INSPIRE].

[74] J. Fiaschi and M. Klasen, Neutralino-chargino pair production at NLO+NLL with resummation-improved parton density functions for LHC Run II, Phys. Rev. D 98 (2018) 055014 [arXiv: 1805.11322] [INSPIRE].

[75] W. Beenakker, M. Klasen, M. Krämer, T. Plehn, M. Spira and P.M. Zerwas, The production of charginos/neutralinos and sleptons at hadron colliders, Phys. Rev. Lett. 83 (1999) 3780 [Erratum ibid. 100 (2008) 029901] [hep-ph/9906298] [INSPIRE].

[76] J. Alwall et al., The automated computation of tree-level and next-to-leading order differential cross sections and their matching to parton shower simulations, JHEP $\mathbf{0 7}$ (2014) 079 [arXiv: 1405.0301] [INSPIRE].

[77] C. Degrande, C. Duhr, B. Fuks, D. Grellscheid, O. Mattelaer and T. Reiter, UFO - The Universal FeynRules Output, Comput. Phys. Commun. 183 (2012) 1201 [arXiv:1108.2040] [INSPIRE].

[78] N.D. Christensen and C. Duhr, FeynRules - Feynman rules made easy, Comput. Phys. Commun. 180 (2009) 1614 [arXiv:0806.4194] [INSPIRE].

[79] ATLAS collaboration, Searches for electroweak production of supersymmetric particles with compressed mass spectra in $\sqrt{s}=13$ TeV pp collisions with the ATLAS detector, Phys. Rev. D 101 (2020) 052005 [arXiv: 1911.12606].

[80] A. Birkedal, K.T. Matchev, M. Perelstein and A. Spray, Robust gamma ray signature of WIMP dark matter, hep-ph/0507194 [INSPIRE].

[81] T. Bringmann, X. Huang, A. Ibarra, S. Vogl and C. Weniger, Fermi LAT search for internal Bremsstrahlung signatures from dark matter annihilation, JCAP 07 (2012) 054 [arXiv:1203.1312] [INSPIRE].

[82] M. Garny, A. Ibarra, M. Pato and S. Vogl, Internal Bremsstrahlung signatures in light of direct dark matter searches, JCAP 12 (2013) 046 [arXiv:1306.6342] [INSPIRE]. 
[83] F. Giacchino, L. Lopez-Honorez and M.H.G. Tytgat, Scalar dark matter models with significant internal Bremsstrahlung, JCAP 10 (2013) 025 [arXiv:1307.6480] [INSPIRE].

[84] T. Toma, Internal Bremsstrahlung signature of real scalar dark matter and consistency with thermal relic density, Phys. Rev. Lett. 111 (2013) 091301 [arXiv:1307.6181] [InSPIRE].

[85] F. Giacchino, L. Lopez-Honorez and M.H.G. Tytgat, Bremsstrahlung and gamma ray lines in 3 Scenarios of dark matter annihilation, JCAP 08 (2014) 046 [arXiv:1405.6921] [INSPIRE].

[86] A. Ibarra, T. Toma, M. Totzauer and S. Wild, Sharp gamma-ray spectral features from scalar dark matter annihilations, Phys. Rev. D 90 (2014) 043526 [arXiv:1405.6917] [INSPIRE].

[87] G.B. Gelmini, P. Gondolo and E. Roulet, Neutralino dark matter searches, Nucl. Phys. B 351 (1991) 623 [INSPIRE].

[88] J. Edsjo and P. Gondolo, Neutralino relic density including coannihilations, Phys. Rev. D 56 (1997) 1879 [hep-ph/9704361] [INSPIRE].

[89] G. Bélanger, F. Boudjema, A. Pukhov and A. Semenov, MicrOMEGAs4.1: two dark matter candidates, Comput. Phys. Commun. 192 (2015) 322 [arXiv:1407.6129] [INSPIRE].

[90] M. Garny, A. Ibarra and S. Vogl, Antiproton constraints on dark matter annihilations from internal electroweak bremsstrahlung, JCAP 07 (2011) 028 [arXiv: 1105.5367] [INSPIRE].

[91] M. Garny, A. Ibarra and S. Vogl, Dark matter annihilations into two light fermions and one gauge boson: General analysis and antiproton constraints, JCAP 04 (2012) 033 [arXiv:1112.5155] [INSPIRE].

[92] A.M. Galper et al., Status of the GAMMA-400 project, Adv. Space Res. 51 (2013) 297 [arXiv: 1201.2490] [INSPIRE].

[93] CTA Consortium collaboration, Design concepts for the Cherenkov Telescope Array CTA: an advanced facility for ground-based high-energy gamma-ray astronomy, Exper. Astron. 32 (2011) 193 [arXiv: 1008.3703] [INSPIRE].

[94] Fermi-LAT collaboration, Searching for dark matter annihilation from Milky Way dwarf spheroidal galaxies with six years of Fermi Large Area Telescope data, Phys. Rev. Lett. 115 (2015) 231301 [arXiv: 1503.02641] [INSPIRE].

[95] L. Bergstrom, T. Bringmann, I. Cholis, D. Hooper and C. Weniger, New limits on dark matter annihilation from AMS cosmic ray positron data, Phys. Rev. Lett. 111 (2013) 171101 [arXiv: 1306.3983] [INSPIRE].

[96] L.A. Cavasonza, H. Gast, M. Krämer, M. Pellen and S. Schael, Constraints on leptophilic dark matter from the AMS-02 experiment, Astrophys. J. 839 (2017) 36 [Erratum ibid. 869 (2018) 89] [arXiv: 1612.06634] [INSPIRE].

[97] AMS collaboration, First result from the alpha magnetic spectrometer on the international space station: precision measurement of the positron fraction in primary cosmic rays of 0.5-350 GeV, Phys. Rev. Lett. 110 (2013) 141102 [INSPIRE].

[98] A. Albert et al., Results from the search for dark matter in the Milky Way with 9 years of data of the ANTARES neutrino telescope, Phys. Lett. B 769 (2017) 249 [Erratum ibid. 796 (2019) 253] [arXiv:1612.04595] [INSPIRE]. 
[99] ANTARES collaboration, Search for dark matter towards the Galactic Centre with 11 years of ANTARES data, Phys. Lett. B 805 (2020) 135439 [arXiv: 1912.05296] [INSPIRE].

[100] ICECuBE collaboration, Search for neutrinos from dark matter self-annihilations in the center of the Milky Way with 3 years of IceCube/DeepCore, Eur. Phys. J. C 77 (2017) 627 [arXiv: 1705.08103] [INSPIRE].

[101] J. Kopp, V. Niro, T. Schwetz and J. Zupan, DAMA/LIBRA and leptonically interacting Dark Matter, Phys. Rev. D 80 (2009) 083502 [arXiv:0907.3159] [INSPIRE].

[102] R. Essig, T. Volansky and T.-T. Yu, New constraints and prospects for sub-GeV dark matter scattering off electrons in Xenon, Phys. Rev. D 96 (2017) 043017 [arXiv:1703.00910] [INSPIRE].

[103] M.K. Pandey et al., Constraints on spin-independent dark matter scattering off electrons with germanium and xenon detectors, arXiv:1812.11759 [INSPIRE].

[104] D. Baxter, Y. Kahn and G. Krnjaic, Electron ionization via dark matter-electron scattering and the Migdal effect, Phys. Rev. D 101 (2020) 076014 [arXiv:1908.00012] [INSPIRE].

[105] DARKSide collaboration, Constraints on Sub-GeV dark-matter-electron scattering from the DarkSide-50 experiment, Phys. Rev. Lett. 121 (2018) 111303 [arXiv:1802.06998] [INSPIRE].

[106] J.D. Lewin and P.F. Smith, Review of mathematics, numerical factors and corrections for dark matter experiments based on elastic nuclear recoil, Astropart. Phys. 6 (1996) 87 [INSPIRE].

[107] XENON collaboration, Dark matter search results from a one ton-year exposure of XENON1T, Phys. Rev. Lett. 121 (2018) 111302 [arXiv:1805.12562] [INSPIRE].

[108] Fermi-LAT collaboration, Search for gamma-ray spectral lines with the Fermi Large Area Telescope and dark matter implications, Phys. Rev. D 88 (2013) 082002 [arXiv:1305.5597] [INSPIRE].

[109] H.E.S.S. collaboration, Search for photon-linelike signatures from dark matter annihilations with H.E.S.S., Phys. Rev. Lett. 110 (2013) 041301 [arXiv:1301.1173] [INSPIRE].

[110] CTA Consortium collaboration, Monte Carlo design studies for the Cherenkov Telescope Array, Astropart. Phys. 43 (2013) 171 [arXiv:1210.3503] [INSPIRE].

[111] XENON collaboration, Physics reach of the XENON1T dark matter experiment, JCAP 04 (2016) 027 [arXiv: 1512.07501] [INSPIRE].

[112] H.H. Patel, Package-X 2.0: A Mathematica package for the analytic calculation of one-loop integrals, Comput. Phys. Commun. 218 (2017) 66 [arXiv:1612.00009] [INSPIRE].

[113] K. Sigurdson, M. Doran, A. Kurylov, R.R. Caldwell and M. Kamionkowski, Dark-matter electric and magnetic dipole moments, Phys. Rev. D 70 (2004) 083501 [Erratum ibid. 73 (2006) 089903] [astro-ph/0406355] [INSPIRE].

[114] E. Masso, S. Mohanty and S. Rao, Dipolar dark matter, Phys. Rev. D 80 (2009) 036009 [arXiv:0906.1979] [INSPIRE].

[115] S. Chang, N. Weiner and I. Yavin, Magnetic inelastic dark matter, Phys. Rev. D 82 (2010) 125011 [arXiv: 1007.4200 ] [INSPIRE].

[116] E. Del Nobile, C. Kouvaris, P. Panci, F. Sannino and J. Virkajarvi, Light magnetic dark matter in direct detection searches, JCAP 08 (2012) 010 [arXiv: 1203.6652] [INSPIRE]. 
[117] C.M. Ho and R.J. Scherrer, Anapole dark matter, Phys. Lett. B 722 (2013) 341 [arXiv: 1211.0503] [INSPIRE].

[118] E. Del Nobile, G.B. Gelmini, P. Gondolo and J.-H. Huh, Direct detection of light anapole and magnetic dipole DM, JCAP 06 (2014) 002 [arXiv: 1401.4508] [INSPIRE].

[119] T. Banks, J.-F. Fortin and S. Thomas, Direct detection of dark matter electromagnetic dipole moments, arXiv:1007.5515 [INSPIRE].

[120] J. Kopp, T. Schwetz and J. Zupan, Global interpretation of direct dark matter searches after CDMS-II results, JCAP 02 (2010) 014 [arXiv:0912.4264] [INSPIRE].

[121] J. Kopp, T. Schwetz and J. Zupan, Light dark matter in the light of CRESST-II, JCAP 03 (2012) 001 [arXiv: 1110.2721] [inSPIRE].

[122] A. Fitzpatrick and K.M. Zurek, Dark moments and the DAMA-CoGeNT puzzle, Phys. Rev. D 82 (2010) 075004 [arXiv: 1007.5325] [INSPIRE].

[123] M.I. Gresham and K.M. Zurek, Light dark matter anomalies after LUX, Phys. Rev. D 89 (2014) 016017 [arXiv:1311.2082] [INSPIRE].

[124] LUX collaboration, First results from the LUX dark matter experiment at the Sanford Underground Research Facility, Phys. Rev. Lett. 112 (2014) 091303 [arXiv:1310.8214] [INSPIRE].

[125] XENON collaboration, Lowering the radioactivity of the photomultiplier tubes for the XENON1T dark matter experiment, Eur. Phys. J. C 75 (2015) 546 [arXiv:1503.07698] [INSPIRE].

[126] P. Barrow et al., Qualification tests of the R11410-21 Photomultiplier tubes for the XENON1T detector, 2017 JINST 12 P01024 [arXiv:1609.01654] [INSPIRE]. 